\title{
Single-cell sequencing characterizes intratumoral profile of immune cells in old mice
} Cangang Zhang ${ }^{1,2 \#}$, Lei Lei ${ }^{1,2,3,4 \#}$, Xiaofeng Yang ${ }^{1,2,3,4 \#}$, Huiqiang Zheng ${ }^{1,2}$, Yanhong Su ${ }^{1,2}$, Anjun Jiao ${ }^{1,2}$, Xin Wang ${ }^{1,2}$, Haiyan Liu ${ }^{1,2}$, Yujing Zou ${ }^{5}$, Lin Shi ${ }^{1,2}$, Xiaobo Zhou ${ }^{1,2}$, Chenming

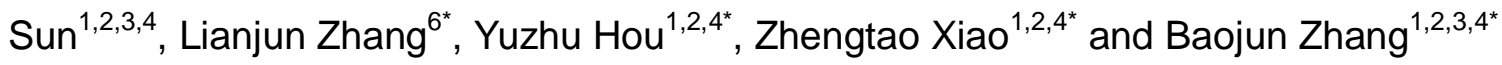

\section{Affiliations:}

${ }^{1}$ Department of Pathogenic Microbiology and Immunology, School of Basic Medical Sciences, Xi'an Jiaotong University, Xi'an, Shaanxi 710061, China

${ }^{2}$ Institute of Infection and Immunity, Translational Medicine Institute, Xi'an Jiaotong University Health Science Center, Xi'an, Shaanxi 710061, China

${ }^{3}$ Key Laboratory of Environment and Genes Related to Diseases (Xi'an Jiaotong University), Ministry of Education, Xi'an, Shaanxi 710061, China

${ }^{4}$ Xi'an Key Laboratory of Immune Related Diseases, Xi'an 710061, Shaanxi, China

${ }^{5}$ Duke University Medical Center, Durham, NC 27705, USA

${ }^{6}$ Suzhou Institute of Systems Medicine, Suzhou, Jiangsu, China; Center for Systems Medicine, Institute of Basic Medical Sciences, Chinese Academy of Medical Sciences and Peking Union Medical College, Beijing, China

\footnotetext{
"These authors contributed equally to this work.
}

“Correspondence to Prof. Baojun Zhang, bj.zhang@mail.xjtu.edu.cn; Prof. Zhengtao Xiao, zhengtao.xiao@xjtu.edu.cn; Prof. Yuzhu Hou, houyuzhu@xjtu.edu.cn; Prof. Lianjun Zhang, zlj@ism.cams.cn 


\begin{abstract}
It has long been thought that aging is a major risk factor for cancer incidence. However, accumulating evidence indicates increased resistance of old animals to tumor growth. A systematic understanding of how old individuals defend against tumor invasion is currently lacking. Here we investigated the differences of age-associated alterations in tumor-infiltrating immune cells between young and old mice using single-cell RNA analysis. Our results showed that a higher proportion of cytotoxic $C D 8^{+} \mathrm{T}$ cells, nTregs, $\mathrm{CDC}$, and M1-type macrophages, while a higher percentage of exhausted CD8 ${ }^{+} \mathrm{T}$ cells, iTregs, $\mathrm{pDC}$, and M2-type macrophages were found in young mice. Importantly, TCR diversity analysis showed top 10 TCR clones consisted primarily of exhausted $\mathrm{CD}^{+} \mathrm{T}$ cells in young mice whereas tip clones were predominantly cytotoxic $\mathrm{CD}^{+} \mathrm{T}$ cells in old mice. Consistently, trajectory inference demonstrated that $\mathrm{CD}^{+} \mathrm{T}$ cells preferentially differentiated into cytotoxic cells in old mice in contrast to exhausted cells in young mice. Meanwhile, we confirmed the main distinctions between young and old mice by flow cytometry. Collectively, our data revealed that a significantly higher proportion of effector immune cells in old mice defend against tumor progression, providing a framework for the immunotherapy of elderly patients with tumors.
\end{abstract}

Keywords: Aging; Tumor microenvironment; Single-cell RNA-Seq; Immune cells; Immunotherapy 


\section{Introduction}

Aging leads to the inevitable time-dependent decline in organ function and is a major risk factor for cancer. Relevant mechanisms of higher cancer incidence in aging individuals include genomic instability, epigenetic changes, loss of proteostasis, and declining immune surveillance (1). Individuals with an aging immune system are more susceptible to infection, and experience reduced vaccine effectiveness, higher incidence of cancer and autoimmune diseases $(2,3)$. More than $50 \%$ of cancers and approximately $70 \%$ of cancer-related deaths occur in patients older than 65 (4). Interestingly, many clinical and preclinical studies indicated that tumor in young patients or animals grow more aggressively than old counterparts (5-7). Furthermore, adoptive transfer of bone marrow or spleen cells from old mice has been shown to reduce the aggressiveness of tumor growth in young mice (5), paradoxical to the concept that cancer is defined as a disease of aging.

Over the past decade, immunotherapies that modulate the immune microenvironment to target and eliminate tumor cells have greatly changed the treatment approaches of cancer (8). The most common treatments that have proven clinical efficacy across a broad range of cancers are immune checkpoint inhibitors against programmed cell death 1(PD1), PDL1, and cytotoxic T lymphocyte-associated antigen 4 (CTLA4), both of which target $\mathrm{T}$ cells to restore their anti-tumor capacity. Immune checkpoint inhibitors significantly improve overall survival(OS) in both young and elder patients, but the magnitude of benefit is age-variable(1). Several clinical studies showed that elderly patients could benefit more from immunotherapies than young patients $(9,10)$ while a similar phenomenon is observed in mice implanted with melanoma tumors $(11,12)$. Therefore, it is of great significance to 
explore the immune system of elderly cancer patients and to further improve immunotherapy for the elderly.

The immune system plays a vital role in recognizing tumor cells and inhibiting the growth of malignant tissues (13). Several studies attempted to reveal the mechanisms of delayed tumor progression in the elderly from the perspective of anti-tumor immunity. It has been shown that an accumulation of $4-1 \mathrm{BBL}^{+} \mathrm{B}$ cells in the elderly generated $\mathrm{GranzymeB}^{+} \mathrm{CD}^{+} \mathrm{T}$ cells controls tumor growth (14), and old $\mathrm{CD}^{+} \mathrm{T}$ cells demonstrate increased adhesion and thus more easily infiltrated tumors through high expression of integrin a 4 (12). However, tumor-infiltrating immune cells form a complex mixture and the dense cross-talk of different types of immune cells plays a critical role in tumor immune surveillance (15). It is difficult to comprehensively characterize the immune profile to fully understand the mechanisms of delayed tumor development and the preferable outcome of immunotherapies in the elderly with conventional immunology technologies. As a revolutionary technology, single-cell RNA sequencing (single-cell RNA seq) allows for the ability to characterize cell types at the single-cell level and accurately define their various immune functions in the complex tumor microenvironment(TME) (16). Accumulating studies using single-cell RNA seq to explore tumor-infiltrating immune cells have been continuously reported (17-21). Besides, Single-cell RNA seq provides a powerful tool to define the $T$ cell receptor (TCR) sequence and dominant clones recognizing tumor antigens (22).

In the current study, we utilized single-cell RNA seq to decipher the transcriptomic landscape of immune cells in B16 melanoma between young and old mice, and uncovered differences in the immune cell proportions and their functional features in young and old mouse tumors. 
We showed that intratumoral immune cells consist of more effector subsets including cytotoxic $\mathrm{CD}^{+} \mathrm{T}$ cells, $\mathrm{CDC}$, and M1-type macrophages in old mice, while immunesuppressive subsets including exhausted $C D 8^{+} \mathrm{T}$ cells, iTregs, pDC, and M2-type macrophages are primarily found in young mice. Importantly, TCR diversity analysis demonstrated that a significant proportion of cytotoxic $\mathrm{CD}^{+} \mathrm{T}$ cells were directly differentiated from effector memory like $C D 8^{+} \mathrm{T}$ cells $\left(C D 8^{+} \mathrm{EM}\right.$ like $\left.\mathrm{T}\right)$ in the TME of old mice. Overall, our data demonstrated that immune cells in the TME of old mice hinder tumor growth, providing critical findings that can facilitate understanding of cancer development and immunotherapy efficacy in elderly patients.

\section{Materials and Methods}

\section{Mice and reagents}

6 to 8 week-old and 20 to 22 month-old female C57BL/6 mice were used in this study. All the mice were obtained from the Laboratory Animal Center of Xi'an Jiaotong University and were housed in the specific pathogen-free animal facility. All animal procedures were performed with the approval of the Animal Care Committee of Xi'an Jiaotong University and conformed to the Guide for the Care and Use of Laboratory Animals published by the US National Institutes of Health.

The antibodies and intracellular staining kit used were as follows: APC/Cy7 anti-mouse Cd4 (clone GK1.5), PE/Cy5 anti-mouse Cd4 (clone GK1.5), APC/Cy7 anti-mouse Cd8a (clone 53-6.7), Pacific BlueTM anti-mouse Cd8a (clone 53-6.7), PE/Cy7 anti-mouse/human Cd44 
(clone IM7), PE/Cy5 anti-mouse Cd25 (clone PC61), PE/Cy5 anti-mouse Cd127(IL-7Ra) (clone A7R34), FITC anti-mouse CD11c (clone N418), FITC anti-mouse/human Cd11b (clone M1/70), PE/Cy7 anti-mouse I-A/I-E (clone M5/114.15.2), APC/Cy7 anti-mouse Cd19 (clone 6D5), Pacific BlueTM anti-mouse/human Cd45R/B220 (clone RA3-6B2), FITC anti-mouse Cd45.2 (clone 104), PE anti-mouse Cd45.2 (clone 104), PE/Cy7 anti-mouse IL-10 (clone JES5-16E3), Pacific BlueTM anti-mouse FOXP3 (clone MF-14), APC/Cy7 anti-mouse IFNy (clone XMG1.2), APC anti-mouse LAP(TGF- $\beta 1$ ) (clone TW7-16B4), APC anti-mouse Cd223(LAG-3) (clone C9B7W), and Fixation Buffer(Cat \# 420801) and Intracellular Staining Perm Wash Buffer(Cat \# 421002). All above-mentioned reagents were purchased from BioLegend (San Diego, CA, USA). FITC anti-Mo Granzyme B (clone NGZB), and Transcription Factor Fixation/Permeabilization Concentrate and Diluent were purchased from eBioscience.

\section{Tumor model and preparation of cell suspensions}

Two groups of mice were injected subcutaneously with $2 \times 10^{5}$ B16F10 cells per mouse. After 5 days, tumor tissues were excised and weighed at indicated time points (every two days). Tumor volumes were determined by caliper measurement using the formula $\mathrm{V}=($ length $\times$ width $^{2}$ ) / 2. Freshly isolated tumor tissues were minced into approximately $1 \mathrm{~mm}^{3}$ cubic pieces and digested using $0.1 \%$ collagenase IV (LS004186, Worthington), $0.002 \%$ DNAse I (D8071, Solarbio), and 0.01\% hyaluronidase (H3506-1G, SIGMA), then incubated on a rocker at $37^{\circ} \mathrm{C}$ for $40-50 \mathrm{~min}$. The digested cells were filtered through a $70 \mu \mathrm{m}$ cell strainer and washed twice with cold FACS buffer. The remaining cells were stained with 
CD45.2-APC (Clone 104; BioLegend) and 7AAD (Part 76332; Lot B226294 Biolegend) for 30 min at $4{ }^{\circ} \mathrm{C}$, then washed and suspended in FACS buffer for flow cytometric sorting using FACS Aria II Cell Sorter (BD Biosciences). Sorted CD45.2 $2^{+}$AAD- cells with a purity greater than $95 \%$ and viability higher than $90 \%$ were used for single-cell RNA sequencing.

\section{Single-cell RNA-seq and VDJ sequencing}

The single-cell library preparation was constructed using 10X Chromium Single Cell V(D)J V2 Reagent Kits according to the manufacturer's protocol. Briefly, single-cell suspensions with a concentration of 1000 cells/ul were loaded on the 10X genomics chromium controller single-cell instrument. Reverse transcription reagents, barcoded gel beads, and partitioning oil were mixed with the cells for generating single-cell gel beads in emulsions (GEM). After reverse transcription reaction, the GEMs were broken. The barcoded, full-length cDNA was amplified and purified to build V(D)J enriched TCR library and 5' gene expression library. The mouse $T$ Cell V (D) J Enrichment Kit was used to isolate and enrich for the $V$ (D) $J$ sequence. Finally, the constructed libraries were sequenced on the Illumina NovaSeq 6000 platform with NovaSeq 6000 S4 Reagent Kit (300 cycles).

\section{Single-cell RNA-seq data processing}

Cell Ranger (v3.0.2,https://support.10xgenomics.com/) was used to process single-cell sequencing data and generate the matrix data containing gene counts for each cell per sample. Briefly, raw base call files from Illumina sequencers were first demultiplexed into FASTQ files with the cellranger mkfastq pipeline. Then, the splicing-aware aligner STAR was 
used to align FASTQs files to the mouse reference genome (mm10). The aligned reads were further counted using the cellranger count pipeline. Finally, the gene expression matrixes of all samples were imported into Seurat v3(23) and merged for subsequent analyses. The following filtering steps were carried out to exclude low-quality cells: cells with fewer than 200 and more than 3000 detected genes were discarded; cells with a high fraction of mitochondrial genes (>10\%) are removed. As a result, a total of 4606 cells (young mice) and 5375 cells (old mice) with 973 informative genes were included in the analyses.

\section{Clustering of single cells and cell-type annotation}

The gene expression data were log-normalized and scaled with default parameters. The top 3,000 most variable genes identified by Seurat function "FindVariableFeatures" were used for the principal component analysis (PCA). The first 50 principal components (PCs) selected based on the ElbowPlot were used for clustering analyses. Cell clusters were identified using FindClusters functions implemented in Seurat with default parameters and resolution parameter as 1.2. The t-SNE and UMAP were used to visualize the clustering results with default parameters and learning rate setting to 1.12. Myeloid cells and lymphocytes were further separated into different subtypes based on the same procedures. The singleR package (v1.4.0)(24) and "MouseRNAData" and "ImmGenData" reference databases were used to annotate the cell type of large cell populations. The cell types of clusters and subclusters were further confirmed and annotated by comparing the specifically expressed genes identified by the Seurat "FindAllMarkers" function with the known cell markers reported in the literature. 


\section{Single-cell trajectory analysis and definition of cell states}

The $\mathrm{CD}^{+} \mathrm{T}$ cell and memory likeT cell subpopulations of interest were selected for single-cell trajectory analysis. Using Monocle (v2.0)(25), cells were ordered according to their inferred pseudotime by following the steps described on Monocle documentation (http://cole-trapnell-lab.github.io/monocle-release/docs/). Only the top 100 differentially expressed genes identified by Seurat were used for dimensionality reduction and trajectory reconstruction. The reduce Dimension function with DDRTree as the reduction method was applied to the top principal components (PCs) and projected the cells onto two dimensions. After the dimension was reduced, the "orderCells" function was used to order cells and the plot_cell_trajectory function was used to visualize the trajectory in two-dimensional spaces.

\section{TCR sequencing data analysis}

The clonotypes of single cells were defined using the Cell Ranger pipeline and default settings. TCR reads were aligned to mm10 reference genome. The consensus TCR annotation was performed using Cell Ranger VDR. Only in-frame rearrangement of TCR alpha and beta chains were considered to be productive and were used to define the dominant TCR of a single cell. Each unique dominant alpha-beta pair was defined as a clonotype. Two cells with identical alpha and beta sequences were assigned to the same clonotype. Cells harboring the clonotype that also presents in other cells were considered as clonal populations, and the number of such cells with the dominant alpha-beta pair indicated the degree of clonality of the clonotype. A total of 699 cells (young mice) and 356 cells (old 
mice) with such TCR alpha-beta pairs in old mice were identified.

\section{GO, KEGG, GSEA and GSVA analysis}

GO and KEGG enrichment analyses were performed by WebGestalt(http://www.webgestalt.org), using genes specifically expressed in TEM-like cells as the input gene list. Gene Set Variation Analysis (GSVA) was used to estimate the enrichment scores of gene sets using the gene count data of each cell and was performed using R package "GSVA" (v1.37)(26). The differential pathways (log2 fold change > 0.32 and adjusted pvalue<0.05) identified by limma package (v3.44) were plotted in the bubble chart. GSEA analysis was performed for each cell subpopulation using the scaled gene expression matrix and $\quad$ GSEA package

available

at https://www.gsea-msigdb.org/gsea/downloads.jsp with default parameters. The gene sets for both GSVA and GSEA are provided by R package msigdbr.

\section{FACS Analysis}

Single cells were obtained from draining lymph nodes and tumors of indicated mice. For cell surface analysis, a total of $1 \sim 5 \times 10^{6}$ cells were stained with Abs in the dark at $4^{\circ} \mathrm{C}$ for $30 \mathrm{~min}$. After washing with cold FACS buffer $(1 \times$ PBS supplemented with $2 \%$ FBS), cells were analyzed using CytoFLEX flow cytometer (BECKMAN COULTER). CytExpert software was used for data analysis. To detect the expression of intracellular transcriptional factors, cells were fixed and permeabilized following 30-minute surface staining according to the manual of Foxp3 kit, followed by anti-Foxp3 antibody staining and FACS analysis. For cytokine 
analysis, cell samples were stimulated in vitro with PMA/lonomycin in the presence of Brefeldin A (BioLegend) and Monensin (BioLegend) for 4 hours. Cells were washed and stained with surface marker antibodies, fixed and permeabilized using Fixation/Permeabilization buffer (BioLegend), and stained with intracellular antibodies.

\section{Quantification and statistical analysis}

Statistical analyses were performed using GraphPad Prism (version 8) and R (version 3.6). Graphs were generated using GraphPad Prism and R ggplot2 package. Statistical analysis was applied to biologically independent mice or technical replicates for each experiment. The two-tailed Student's t-test was used for all statistical calculations using GraphPad Prism 8 software. All error bars were reported as mean \pm SEM with $n=3$ independent biological replicates. The level of significance is indicated as ${ }^{*} P<0.05,{ }^{* *} P<0.01,{ }^{* *} P<0.001,{ }^{* * *} P$ $<0.0001$. 


\section{Results}

\section{Changes in immune cell composition of the TME during aging}

To assess the effects of young and old host immune environment on tumor growth, we established syngeneic tumor models by subcutaneously implanting B16F10 melanoma cells into the right flanks of young (8-10weeks, $n=3$ ) and old (20-22months, $n=3)$ C57BL/6J mice. Consistent with published results $(5,12,27)$, old mice underwent significantly delayed tumor growth compared to young mice (Fig. 1A), indicating that host-intrinsic factors influenced tumor growth. To reveal the tumor-infiltrating immune cell composition, we profiled single-cell transcriptomes of $\mathrm{CD} 45^{+}$immune cells paired with TCR sequences of $\mathrm{T}$ cells in tumors isolated from young and old mice (Fig. 1B). After quality control and filtering, we obtained single-cell transcriptomes from 9,981 cells (4,606 for young mice and 5,375 for old mice) with a median of 973 genes (Methods). The single-cell RNA-seq (scRNA) data was normalized together and no batch effects were detected by PCA analysis (Fig. S1A). The clustering result was neither predominately contributed by cell cycle phases (Fig. S1B) nor by the sample group (Fig. S1C). Clustering analysis revealed 25 cell populations in the tumors of young and old mice. All clusters, except for cluster 25 expressing a high level of Ptprc, were identified as immune cells and used for subsequent analyses (data not shown). Analysis of the top 20 differentially expressed genes across these clusters revealed 10 cell types with unique transcriptional features (Fig. 1C, Supplemental data 1), including macrophages (Adgre1, Cd14 and Fcgr3), conventional dendritic cells (Xcr1, Flt3 and Ccr7), plasmacytoid dendritic cells (Siglech, Clec10a and Clec12a), monocytes (Ly6c2 and Spn), neutrophils (Csf3r, S100a8 and Cxc/3), natural killer cells (Gzma, Klra4 and Nkg7), 
$\mathrm{CD}^{+}{ }^{+} \mathrm{CD} 4^{-} \mathrm{CD}^{-} \mathrm{T}$ cells $(C d 3 d, \mathrm{Cd} 3 e$ and $\mathrm{Cd} 3 g), \mathrm{CD}^{+} \mathrm{CD} 4^{-} \mathrm{T}$ cells $(\mathrm{Cd} 3 d, \mathrm{Cd} 3 e$ and $\mathrm{Cd} 8)$, CD4 $^{+}$CD8 - T cells (Cd3g, Cd4 and Ctla4), and B cells (Cd79a, Cd79b and Cd19) (Fig. S1D). We visualized these cell types in two-dimensional spaces using t-distributed stochastic neighbor embedding (tSNE) and confirmed their cell identities by examining the expression of classic marker genes curated from the literature (28-30) (Fig. S1E). The distribution of cell types captured by scRNA was comparable between young and old mice (Fig. 1D). However, the proportions of $\mathrm{CD}^{+} \mathrm{CD} 4^{-} \mathrm{CD} 8^{-} \mathrm{T}$ lymphocytes, $\mathrm{B}$ lymphocytes, $\mathrm{pDC}$, and neutrophils were dramatically reduced, whereas the proportions of macrophages, cDCs, and $\mathrm{CD}^{+} \mathrm{CD} 8^{-}$ T lymphocytes were increased in old mice compared to young mice (Fig. 1E), highlighting the significant differences of tumor-infiltrating immune cells during aging.

\section{Percentage comparison within tumor-infiltrating myeloid cells between young and old mice}

Since myeloid cells were the most abundant cell type in our dataset, we first investigated the heterogeneity of myeloid cells in tumors of young and old mice through finer clustering. Although the size of total myeloid population was similar between young and old mice, old mice had significantly lower percentage of neutrophils but higher percentage of DCs in old mice in comparison to that of young mice. The proportions of tumor-infiltrating monocytes and macrophages remained unchanged (Fig. 2A). To assess if neutrophils had potential functional differences between young and old mice, we compared gene expression across two groups (Fig. S2A). Most genes displayed equivalent expression levels between young and old mice, suggesting that aging didn't affect the functional activity of neutrophils which 
mainly relied on infiltration and cytokine production. Other myeloid cells, including macrophages, DCs, and monocytes, which consist of diverse subtypes $(31,32)$, were then partitioned into 5, 4, and 2 subsets, respectively (Fig. 2B, S2B-C). We confirmed their identities by examining the expression of classic markers $(29,33)$ (S2D-E, Supplemental data 2). The subsets that belong to the same cell type were clearly clustered together. Each subset was characterized by a specific gene expression pattern (Fig. 2C) and was labeled using their cluster index. Among them, the subtypes of macrophages and DCs showed striking differences in their proportions (Fig. 2D). We thus performed elaborate analyses on their cell subgroups next.

\section{Macrophage subsets in tumors of young and old mice}

Macrophages are highly plastic cells that divide into diverse functional subsets in tumor enviroenment $(31,34)$. To understand the presence of macrophage subsets and their potential functions in the tumor immune microenvironment of old mice, we first evaluated the expression of classical M1-like and M2-like macrophage lineage markers in tumor-infiltriting macrophages (Fig. 3A). Most M1-like macrophage markers (e.g. inflammatory genes), including Cd86, Cxc/10, Cc/9, and Ly6a, were highly expressed in MC1, whereas the M2-like macrophage markers, i.e., Mrc1 and Arg1 were highly expressed in M2 and M4. All subsets showed considerable differences between two groups (Fig.3B). The most striking observation was the accumulation of MC1 in tumors of old mice in contrast to highly enriched MC2 and MC3 in tumors of young mice.

The chemokine system deeply affects activation, chemotaxis, and polarization of 
macrophages $(35,36)$. We found that each macrophage subset had a distinct chemokine expression profile (Fig.3C). MC1 expressed a high level of $\mathrm{CxCl10}$ which may allow cells to attract both dendritic cells and T lymphocytes via chemotaxis $(37,38)$. MC2 highly expressed Ccl4, Ccl6, and Cxcl13; MC3 highly expressed Ccr2; MC4 highly expressed Ccl8, Cc/12, Cxcl16, and Vcam1 which was associated with tumor progression (39); MC5 highly expressed Cc/3, Cxcr4, and integrin Itga4 (also known as Cd49d), which are specifically expressed in the monocyte-derived TAMs (40). Moreover, we found that a large proportion of MC1 displayed the highest inflammatory, macropinocytotic and phagocytotic activities among all macrophage subtypes in old mice (Fig.3D, Supplemental data 3). Interestingly, MC3 showed mild activity scores for most macrophage-associated pathways (Fig.3D). Several genes including Maf, II4ra, Ly6c2, and C1qa were significantly different in MC2 of young mice compared to old mice (Fig.S3B). Both MC2 and MC4 in old mice expressed higher Vegfa (Fig.S3B, S3C), which suggests that decreased angiogenesis in tumors of old mice was not due to decreased VEGFA production. Overall, we demonstrated that macrophages in old mice acquire increased pro-inflammatory states.

\section{Dendritic cell subtypes in tumors of young and old mice}

Dendritic cells (DCs) are also heterogeneous and display various functional states in the TME (41). Four DC subsets were identified based on known biomarkers and genes critical for DC function (Fig. 4A). DC1 was identified as Itgax ${ }^{+} \operatorname{tgam}^{+}$Cd14 $4^{+}$Monocyte-derived

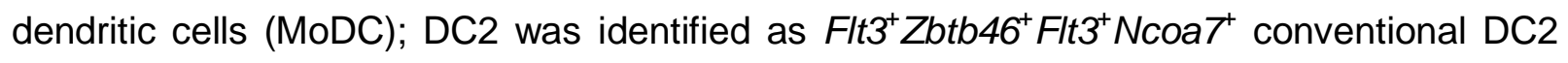
(cDC2); DC3 was defined as pDC with Siglech ${ }^{+}$Irf8 $\mathrm{Clec12a}^{+}$; DC4, which shared several 
common biomarkers with DC2 but specifically expressed Clec9a, Btla, Xcr1, and Itgae, was identified as cDC1. cDC2 and MoDC were highly enriched in tumors of old mice, while the pDC was highly enriched in tumors of young mice (Fig. 4B). We then determined the differential expression of genes relevant to cytokines production and chemotaxis, as well as those critical for antigen processing and presentation, and immune regulators, which allow DCs to regulate immune responses (Fig. 4C, S4A, and S4B). We showed that chemokine receptor CCR7, a member of the G protein-coupled receptor family responsible for the recruitment of lymphocytes and mature DC to lymphoid tissues, was highly enriched in MoDC; Both cDC1 and cDC2 highly expressed co-stimulatory genes Cd80, Cd86, Cd83 and/or Ly6a, indicating their roles in activating local immune response. pDC highly expressed Cd37, Lag3, Sla2, and Lair1, suggesting their inhibitory roles in downregulating local immune response (Fig. 4C). We further confirmed the changes in pDC proportions by verifying the expression of Lag3 in two groups using an independent cohort in both dLNs (Fig. 4D) and tumor tissues (Fig. 4E) of young and old mice. In addition, we found that high expression of the $\mathrm{H} 2$ genes in $\mathrm{CDC} 1$ and MoDC suggests enhanced antigen presentation capacity (Fig. 4SC). To further explore the differences in subset proportions and potential functions between young and old groups, we performed gene GO analyisis on gene sets related to DCs (Fig. 4F, Supplemental data 3). We found that while pDCs in young mice produced larger quantities of cytokines, $\mathrm{CDC} 1$ and MoDC in old mice also tended to secrete more cytokines. cDC2 in old mice significantly expressed a batch of genes enriched in processes critical for dendritic cell differentiation, chemotaxis, positively regulation of antigen processing and presentation, while downregulating genes associated with dendritic cell 
apoptotic process. MoDC in old mice presented relatively higher ability in cytokine production, antigen presentiation and survival compared to that in young mice. Both Fcgr1 and Tcf4 genes, which are associated with immunoglobulin production, had higher expression levels in MoDC of old mice. In contrast, Cd37, which is associated with negative regulation of myeloid dendritic cell activation(42), was highly expressed in MoDC of young mice (Fig. 4G). Overall, these findings demonstrated that cDCs and MoDCs are prodominent populations in old mice supporting antitumor responses.

\section{Characterization of $\mathrm{T}$ cell subtypes in tumors of young and old mice}

$\mathrm{T}$ cells are the main effector cells of adaptive immunity mediating antitumor response and their function is dependent on differentiation into distinct subsests (43). We extracted 2,934 T cells (1445 in young mice and 1489 in old mice) and performed unbiased clustering using highly variable genes to reveal 9 clusters of T cell populations. The clusters were visualized using uniform manifold approximation and projection (UMAP; Fig5A). Each subpopulation was annotated based on the expression levels of the classical T cell markers (Fig. 5B, S5A) and regulators related to cell differentiation and function (Fig 5C, S5B). The universal T cell markers, e.g. Cd3e, $C d 3 d$, were highly expressed in all subpopulations. Four clusters highly expressing Cd8a gene were assigned as $\mathrm{CD}^{+} \mathrm{T}$ cells, including activated_ $\mathrm{CD}^{+} \mathrm{T}$ $\left(\mathrm{Cd}_{\left.69^{+} \mathrm{Cd} 28^{+}\right), \quad \text { exhausted_CD8 }}{ }^{+} \quad \mathrm{T} \quad\left(\mathrm{Gzmb}^{+}\right.\right.$Prf1 $^{+} \mathrm{Pdcd}^{+}$Lag3 $^{+}$Tigit $^{+}$Havcr2 $\left.^{+}\right)$, cytotoxic_CD8 ${ }^{+} \mathrm{T}\left(\mathrm{Gzmk}^{+} \mathrm{Gzmb} b^{+} \mathrm{Pdcd} 1^{+}\right)$, and Clec4e_CD8 ${ }^{+} \mathrm{T}\left(\mathrm{Clec} 4 \mathrm{e}^{+}\right)$; two clusters were assigned as effector memory like (EM_like) with high expression of genes such as Cd44, Cd69, including CD8 ${ }^{+}$EM_like T with expression of Gzmk and Cd8a, and CD4 ${ }^{+}$EM_like T 
with expression of Gzmb and Cd4; The rest of clusters were assigned as naive_T with high expression of naïve gene markers Ccr7, Sell, Tcf7 and Lef1, memory-like T cells (Memory_like T; II7r $r^{+} \cos ^{+}$Gzmk Gzmb) and regulatory T cells (Treg; Cd4 ${ }^{+}$Foxp3 $3^{+}$) (Fig. S5C). The density distributions reflected difference in population frequency (Fig. 5A, Supplemental data 4). Old mice exhibited higher proportions of cytotoxic_ CD8 ${ }^{+} \mathrm{T}_{\text {, CD8 }}{ }^{+}$ EM_like T, CD4 ${ }^{+}$EM_like T, and memory_like T but lower proportions of activated_ CD8 ${ }^{+} \mathrm{T}$, exhausted_ CD8 ${ }^{+} \mathrm{T}$, and naive_T compared with young mice (Fig. 5D). No obvious differences were observed in the proportions of Treg and Clec4e_CD8 ${ }^{+} \mathrm{T}$ cells between the two groups. In general, Tregs can be divided into two types with distinct phenotype and function, including naturally occurring Tregs (nTregs) and induced Tregs (iTregs). GSEA showed more genes associated with iTregs within tumors of young mice compared to old mice (Fig. S6A). Consistently, FACS analysis of Tregs in tumors of old mice revealed higher proportion of Helios ${ }^{+}$cells (Fig. S6B) and higher level of Helios expression compared to that of young mice (Fig. S6C). Ccr7, controling thymic Tregs recirculation, was highly expressed in old mice (Fig. S6D). TGF- $\beta$, essential for Tregs to exert suppressive function (44), was also highly expressed in Tregs of old mice. Flow cytometry confirmed that old mice had significantly higher percentages of TGF- $\beta^{+}$Tregs in both dLNs (Fig. S6E) and tumor tissues (Fig. S6F). Clearly, more CD8 ${ }^{+}$effectors and similar Tregs were present in the TME of old mice.

\section{Striking shift of tumor-infiltrating $\mathrm{CD}^{+} \mathrm{T}$ subsets between young and old mice}

To gain deeper insights into $\mathrm{CD}^{+} \mathrm{T}$ cell features in tumor environment of old mice, we 
further divided total $\mathrm{CD}^{+}$cells into four groups, including activated, cytotoxic, exhausted, and Clec4e positive $\mathrm{CD}^{+} \mathrm{T}$ cells. There was a higher proportion of cytotoxic $\mathrm{CD} 8^{+} \mathrm{T}$ cells in old compared to young mice while there were more activated $\mathrm{CD}^{+} \mathrm{T}$ cells and exhausted $\mathrm{CD}^{+} \mathrm{T}$ cells in young mice (Fig. 6A). Flow cytometry analyisis confirmed that old mice had lower percentages of PD $-1^{+} \mathrm{LAG}^{+}$and $\mathrm{Tim}-3^{+} \mathrm{LAG} 3^{+}$(exhausted) cells (Fig. 6B), as well as higher percentages of IFN- $\mathrm{Y}^{+}$and Granzyme $\mathrm{B}^{+}$(cytotoxic) cells in tumors (Fig. 6C). This result was also confirmed by the TCR repertoire analysis, where the top 10 clones in young mice were annotated as exhausted $\mathrm{CD}^{+} \mathrm{T}$ cells and the top 10 clones in old mice were annotated as cytotoxic $\mathrm{CD}^{+} \mathrm{T}$ cells (Fig. 6D, Supplemental data 5). To elucidate transcriptional fate of effector $\mathrm{CD}^{+} \mathrm{T}$ cells, we performed trajectory analysis on activated, cytotoxic, and exhausted CD8 ${ }^{+}$T cells (Fig. 6E). The trajectory branch started with activated $\mathrm{CD}^{+}$cells and ended with cytotoxic and exhausted $\mathrm{CD}^{+}$cells (Fig 6F, Fig S7B), suggesting activated $\mathrm{CD}^{+} \mathrm{T}$ cells subsequently differentiated into cytotoxic or exhausted states. Moreover, we found that most genes associated with T cell exhaustion were enriched in young mice while the genes associated with $\mathrm{T}$ cell activation were enriched in old mice (Fig. S7C). Cytotoxic population were enriched in cell proliferation-related pathways in old mice whereas apoptosis process-associated pathways were enriched in young mice (Fig. S7D). The exhausted population expressed cell adhesion-related genes in old mice whereas pathways negative regulating proliferation were enriched in young mice (Fig. S7E). Consistent with pathway enrichment analysis, activated and exhausted $\mathrm{CD}^{+} \mathrm{T}$ cells in young mice displayed significantly higher apoptosis score (Fig. S7F, Supplemental data 3), suggesting effector T cells are more prone to death. Moreover, we analyzed shared TCR of 
exhausted population by all other $\mathrm{CD}^{+} \mathrm{T}$ cells, including activated CD8 ${ }^{+} \mathrm{T}$, cytotoxic_ CD8 ${ }^{+}$ T, and $\mathrm{CD}^{+}$EM_like T (Fig. 6G). The statistical results showed that tumor-infiltrated exhausted $\mathrm{CD}^{+} \mathrm{T}$ in young mice shared more TCR clones with activated_CD8 ${ }^{+} \mathrm{T}$ and cytotoxic_CD8 ${ }^{+} \mathrm{T}(\mathbf{F i g} .6 \mathrm{H})$, suggesting that $\mathrm{CD}^{+} \mathrm{T}$ cells in young mice are more prone to exhaustion.

\section{Characterization of tumor-infiltrating $\mathrm{CD}^{+}$memory like $\mathrm{T}$ cells in young and old mice}

In line with the previous reports that the number of memory $\mathrm{T}$ cells increase during aging in both human (45) and mice (46), we found that old mice exhibited higher proportions of CD8+ EM_like T CD4 ${ }^{+}$EM_like T, and memory_like T cells than young mice, as well as lower proportions of naive_T cells (Fig. 7A). Flow cytometry analysis confirmed more CD8 ${ }^{+}$ TEM(Fig. 7B) and CD4 ${ }^{+}$TEM (Fig. 7C) cells in both dLNs(upper pannel) and tumor tissues(lower pannel) of old mice. While the expression of memory markers were similar, $\mathrm{CD}^{+}$EM_like T cells expressed higher levels of Cd28, Ly6a and Gzmk and lower levels of Cd69, Fcer1g, and Cd7 in old mice compared to young mice (Fig. S8A). Similarly, CD4 ${ }^{+}$ EM_like T cells expressed inflammation-related genes including Nfkb1, Isg15, and Jund in old group, whereas immunosuppressive genes including Tigit, and glycolysis-related gene Aldoa in young group (Fig. S8B).

In addition to $\mathrm{CD}^{+}$and $\mathrm{CD}^{+} \mathrm{EM}$ like T cells, we also discovered an undefined type of memory_like T cells, expressing genes related to T cell activation and differentiation (Fig. 7D). Apart from highly expressing costimulatory genes (Cd82, Icos, Ly6a, Cd44), memory_like T cells also expressed high level of differentiation-related genes (Rora, Cebpb, 
Zeb1 and Ikzf3), and proliferation-related genes (Cdkn1a, Bcl2a1d, Bcl11b and Zc3h12a) (Fig. 7E).

To further explain the differentiation relationship among $\mathrm{CD}^{+} \mathrm{T}$ cell populations, we constructed a trajectory tree for $\mathrm{CD}^{+} \mathrm{EM}$ _like T, cytotoxic_CD8 ${ }^{+} \mathrm{T}$ and exhausted_CD8 ${ }^{+} \mathrm{T}$ cells. CD8 ${ }^{+}$EM_like $T$ cells were located at the beginning of the trajectory tree based on pseudotime (Fig. S8C), while cytotoxic_CD8 ${ }^{+} \mathrm{T}$, and exhausted_CD8 ${ }^{+}$T cells were located in the middle and ends of the trajectory tree, respectively (Fig. S8D). CD8 ${ }^{+}$EM_like T of old mice were activated to become cytotoxic_CD8 ${ }^{+}$T earlier than that of young mice. In contrast, cytotoxic_CD8 ${ }^{+} \mathrm{T}$ of young mice underwent exhaustion earlier and more readily than that of old mice (Fig. 7F).

\section{Discussion}

In this study, we reported the profile of immune cells in the TME of young and old hosts at the single-cell level and revealed possible immune mechanisms for old individuals that underwent delayed tumor progression. Given that most preclinical models used young animals, and most common cancers behaved differently in young and elderly patients, we and others (47-50) considered using old animals to explore the role of aging in the tumor immune microenvironment. Although the life span of mice and humans are very different, many studies have shown that humans and mice are very similar in disease progression, the physiological functions of various organs, and the molecular mechanism of aging $(51,52)$. In mice, senescence begins at least at 18 months. Therefore, we selected mice that are 20 to 22 month-old as the old group and 8 to 10 week-old mice as the young group. After 
confirming delayed tumor growth in old mice (47), we sorted CD45 ${ }^{+}$immune cells with flow cytometry before performing single-cell sequencing analysis. Our data showed that immune cells were distinct in composition and characteristics between these two groups, supporting that in comparison to young mice, old mice present a tumor environment with effector populations with stronger functional features to better defend against tumor progression.

T cell-mediated immunity is a vital component of the immune response for host defense against cancer (48). $\mathrm{CD}^{+} \mathrm{T}$ cells, the major cytotoxic killers of tumor cells, exhibited heterogeneity in the TME by single-cell sequencing $(19,28,53,54)$. Here, we aimed at investigating tumor-infiltrating $\mathrm{T}$ cell populations and the corresponding characteristics, and determining the clonal dominance of $\mathrm{CD}^{+}$TILs in B16 melanoma tumor model. 9 types of T cells were characterized based on transcriptome analysis of single cells, including 4 types of $\mathrm{CD}^{+} \mathrm{T}$ cells, 3 types of memory like T cells, naïve T cells, and Tregs. We found that a large proportion of tumor-infiltrating $\mathrm{CD}^{+} \mathrm{T}$ cells were newly activated but exhausted in young mice, whereas a significantly higher proportion of cytotoxic or memory T cells are present in old mice. Flow cytometry further confirmed that old mice had higher percentages of IFN- $\mathrm{Y}^{+}$ and Granzyme $\mathrm{B}^{+} \mathrm{CD}^{+} \mathrm{T}$ cells in both dLNs and tumor tissues. Consistently, a recent study reported that $\mathrm{Cd} 49 \mathrm{~d}^{\mathrm{hi}} \mathrm{Cd} 8^{+}$cells are more abundant in tumors and exhibit a stronger anti-tumor effect in old mice (12). In addition, tumor-infiltrating $\mathrm{CD}^{+} \mathrm{T}$ cells showed enhanced survival in old mice, while those in young mice were more prone to apoptosis. The trajectory analysis of activated_ CD8 ${ }^{+} \mathrm{T}$, cytotoxic_CD8 ${ }^{+} \mathrm{T}$, and exhausted_ CD8 ${ }^{+} \mathrm{T}$ cells showed that tumor-infiltrating $\mathrm{CD}^{+} \mathrm{T}$ cells underwent two sequential stages following activation with continuing differentiation trajectory in young mice, whereas $\mathrm{CD}^{+} \mathrm{T}$ cells were 
primarily located at the end of the trajectory tree in old mice, suggesting that effector $\mathrm{CD}^{+} \mathrm{T}$ cells underwent distinct differentiation programming during defense against tumor cells in the TME of young versus old mice.

With aging, continuous antigen stimulation and degeneration of the thymus cause the distribution of $\mathrm{T}$ cell subsets to shift from naive $\mathrm{T}$ cells to memory or memory-like cells (55). Previous studies demonstrated a reduction of response to neoantigens in elderly individuals due to a lower number of naive T cells (48). However, this does not necessarily indicate that the ability of old individuals to defend against foreign antigens is reduced since memory $\mathrm{T}$ cells cross-reactive to neoantigens may directly differentiate into effector T cells, and secrete inflammatory cytokines together with bystander memory-like cells. Consistently, there are several subsets of memory-like cells that exhibit stronger effector function. In old mice, CD8 ${ }^{+}$ EM_like T expressed higher levels of costimulatory genes: Ly6a, Cd28, and the cytotoxic gene Gzmk, while CD4 ${ }^{+}$EM_like T expressed higher levels of inflammation-related genes: Nfkb1, Isg15, and Jund. In contrast, CD4 ${ }^{+}$EM_like T cells in young mice expressed higher inhibitory genes: Tight, and Aldoa. In addition, we also identified a unique population, Memory_like T cells, which highly expressed II7r, Cd44, and co-stimulation genes (e.g. Cd82, Icos, Ly6a). Gene expression enrichment analysis showed that upregulated genes in these cells were related to cell differentiation, proliferation, and T cell activation, suggesting that this cluster of unique $\mathrm{T}$ cells in the elderly represent recently activated effector cells derived from memory T cells. Analysis of shared TCR clones between T cell populations in different states can be used to infer differentiation and cell origin(56). Compared with the old group, CD8 ${ }^{+}$EM_like T, cytotoxic_CD8 ${ }^{+}$T cells, and exhausted_CD8 ${ }^{+} \mathrm{T}$ cells in the young 
group shared a higher proportion of TCR clones. The relationships among 3 subpopulations were further confirmed by trajectory analysis.

NK cells are another key player of immune surveillance against tumorgenesis. However, different from the general decline of T cells with aging(57), the proportion and function of NK cells remained relatively stable. Although GSEA showed that NK cells of young mice were relatively active(Fig. S9A), many functions showed no difference except for pathways related to metabolism (Fig. S9B). Our single-cell data demonstrated that tumor-infiltrating NK cells expressed similar levels of functional molecules, such as Prf1, Gzmb, Nkg7, NKG2D(KIrk1), Ly-49g(Klra7), and CD94(KIrd1) in both young and old hosts (Fig. S9C). Consistent with a previous report in humans(58), our data also showed that NK cells in old mice expressed a lower level of $N K G 2 A(K I r c 1)$ but a higher level of $L y 49(K I r a 4)$ (Fig. S9C). In addition, we found that NK cells in old mice expressed higher levels of inflammatory genes (Nfkb1 and Stat3) and activation molecule 4-1BB (59), indicating stronger effector function of NK cells and the proinflammatory status in the elderly.

Previous studies show that B cells are generated at a reduced rate(60), showing lower levels of percentage and absolute numbers $(61,62)$ while also differentiating into longer-lived memory B cells(62) in old individuals. In the tumor environment, our data also showed that the infiltrating B cells in the tumors of old mice tended to be memory B cells (Fig. S10A), and were present at a lower percentage than that in young mice (Fig. S10B). In addition, tumor-infiltrating B cells of old mice expressed lower levels of canonical B cell markers (Cd19, Cd79a, and Cd79b) compared to that of young mice (Fig. S10C). These cells were similar to a type of exhausted or "double negative" memory B cells expressing low level of 
$\lg \mathrm{D}$ and $\mathrm{Cd} 27$ reported in a previous study (63). IgM memory B cells accumulate with age and become the predominant memory B cell subset. Consistently, our data showed that tumor-infiltrating B cells in old mice express higher level of IgM but lower lgD expression. Previous studies found that 4-1BBL-expressing B cells increase with age in humans and mice, enhancing Granzyme $\mathrm{B}$ expression of $\mathrm{CD}^{+} \mathrm{T}$ cells by presenting endogenous antigens(12), however, the current study did not reveal an enrichment of this population in the tumor of old mice.

Among myeloid cell lineages, cDCs and M1-type macrophages play positive roles in defending against tumor growth through innate mechanisms and activation of adaptive immunity $(31,32)$. CDC1 mainly activates $\mathrm{CD}^{+} \mathrm{T}$ cells by processing and presenting exogenous antigens through MHC class I molecules, while cDC2 is crucial for inducing the immune response of $\mathrm{CD}^{+} \mathrm{T}$ cells $(32,64)$. Our data also showed that the proportion of dendritic cells that produce the inflammatory factor (TNF- $\alpha$ ), and $C c / 5$, critical for cDC1 recruitment to $\operatorname{TME}(65)$, were increased in old mice. $\mathrm{cDC} 2$, vital to stimulating $\mathrm{Cd}^{+} \mathrm{T}^{+}$ cell-mediated immunity in cancer (66), had similar abilities of antigen processing and presentation in young and old mice, but a higher population of cDC2 in old mice. M1-type macrophages express higher inflammatory genes and are associated with antigen presentation(34,67). We showed that a higher percentage of macrophages in the tumor of old mice are M1-type with enhanced inflammatory factor expression (TNF- $\alpha$ ) and potential costimulatory function ( $\mathrm{Cd80}, \mathrm{Cd} 86)$. Previous studies showed that M1-type macrophages in old mice display powerful phagocytosis and micropinocytosis abilities, which are beneficial to the internalization of exogenous antigen(68). 
In parallel to effector cells playing positive roles in mediating anti-tumor response, there are well-identified immune cells that negatively regulate immune response, including Tregs, MDSCs, M2-type macrophages, and pDCs in tumor environment(69,70). Previous studies revealed that elderly individuals demonstrated changes in Tregs' frequency and function with aging $(71,72)$. In contrast, the current study showed that the proportion and functional gene expression of Tregs in old mice were similar to that in Tregs of young mice. However, tumor-infiltrating Tregs tended to be of thymus origin (nTregs) in old mice whereas more are iTregs in young mice, supported by flow cytometry analysis showing higher expression of Helios in Tregs from old mice. In addition to Tregs, MDSCs are specialized immunosuppressors to prevent excessive inflammation $(73,74)$ and promote tumor growth and metastasis(33). However, no MDSCs were identified in the current data. M2-type macrophages are characterized by high expression of Arg1, Vcam1, or Cd206, and are associated with immunosuppression (75) and tumor growth (31). We identified two types of macrophages with high expression of $\operatorname{Arg} 1(\mathrm{MC} 2)$ and high expression of Vcam1(MC4), and both groups of macrophages were elevated in young mice. pDCs have been shown to associated with poor patient prognosis in several cancers by inducing the expansion of Tregs(76). pDCs were present a lower level in old mice, potentially allowing for more robust anti-tumor immunity.

In conclusion, we utilized single-cell RNA sequencing analysis to evaluate the proportion and gene expression difference of immune cell components in the tumors of young and old mice. In comparison to young mice, old mice showed a higher proportion of effector cells (cytotoxic T cells, cDC, and M1-type macrophages) and a lower proportion of dysfunctional 
and immunosuppressor cells (exhausted T cells, iTregs, pDC, and M2-type macrophages) in the TME. TCR spectrum and trajectory inference analysis demonstrated preferential differentiation of $\mathrm{CD}^{+} \mathrm{T}$ cells into cytotoxic cells in old mice versus exhausted cells in young mice. However, the underlying mechanisms that regulate distinct differentiation paths requires further investigation.

\section{Code availability}

The codes generated in this study are available at the Github repository (https://github.com/Immugent/single-cell-data-process)

\section{Data Availability Statement}

The raw data will be made available by the authors, without undue reservation, to any qualified researcher.

\section{Ethics Statement}

The animal study was reviewed and approved by the Animal Care Committee of Xian Jiaotong University.

\section{Author Contributions}

B.Z. and C.Z. conceptualized the study and designed experiments. C.Z. and H.Z. collected the tumor samples with help from L.L., Y.S., H.L, and X.W. L.S., A.J., Y.Z. and C.Z. prepared samples for single-cell sequencing and performed experiments with help from X.Y. and Y.S. 
C.Z. analyzed scRNA-seq data with input from Z.X. C.Z. and B.Z. wrote the manuscript. B.Z., Z.X., C.S., X.Z. and Y.H. reviewed and revised the manuscript.

\section{Fundings}

This work was supported by grants from Major International (Regional) Joint Research Project (81820108017, B.Z.), Natural Science Foundation of China (81771673, B.Z.), Young Talent Program of Xi'an Jiaotong University (YX1J005, B.Z.), COVID-19 special project of Xi'an Jiaotong University Foundation (xzy032020002, B.Z.), COVID-19 special project funded by Qinnong Bank and Xi'an Jiaotong University (QNXJTU-01, B.Z.), Natural Science Foundation of Shaanxi Province (2020JM-065, X.Z.; 2020JQ-098, L.L.), and project funded by China Postdoctoral Science Foundation (2019M653673, X.Y.).

\section{Acknowledgments}

We thank Dr. Guohua Zhang from Beckman Coulter, Prof. Chen Huang and Dr. Xiaofei Wang from Department of Cell Biology and Genetics for flow cytometric technical supports.

\section{Declaration of interests}

The authors declare no competing interests.

\section{References}

1. Fane M, Weeraratna AT. How the ageing microenvironment influences tumour progression. Nature reviews Cancer 2020;20(2):89-106 doi 10.1038/s41568-019-0222-9.

2. Montecino-Rodriguez E, Berent-Maoz B, Dorshkind K. Causes, consequences, and reversal of 
immune system aging. The Journal of clinical investigation 2013;123(3):958-65 doi 10.1172/JCI64096.

3. Goronzy JJ, Weyand CM. Understanding immunosenescence to improve responses to vaccines. Nature immunology 2013;14(5):428-36 doi 10.1038/ni.2588.

4. Shanmugham LN, Petrarca C, Frydas S, Donelan J, Castellani ML, Boucher W, et al. IL-15 an immunoregulatory and anti-cancer cytokine. Recent advances. Journal of experimental \& clinical cancer research : CR2006;25(4):529-36.

5. Ershler WB, Moore AL, Shore H, Gamelli RL. Transfer of age-associated restrained tumor growth in mice by old-to-young bone marrow transplantation. Cancer research 1984;44(12 Pt 1):5677-80.

6. Diab SG, Elledge RM, Clark GM. Tumor characteristics and clinical outcome of elderly women with breast cancer. J Nat/ Cancer Inst 2000;92(7):550-6 doi 10.1093/jnci/92.7.550.

7. Ershler WB, Longo DL. Aging and cancer: issues of basic and clinical science. $J$ Nat/ Cancer Inst 1997;89(20):1489-97 doi 10.1093/jnci/89.20.1489.

8. Sharma P, Allison JP. Immune checkpoint targeting in cancer therapy: toward combination strategies with curative potential. Cel/2015;161(2):205-14 doi 10.1016/j.cell.2015.03.030.

9. Elias R, Karantanos T, Sira E, Hartshorn KL. Immunotherapy comes of age: Immune aging \& checkpoint inhibitors. J Geriatr Onco/2017;8(3):229-35 doi 10.1016/j.jgo.2017.02.001.

10. Elias R, Hartshorn K, Rahma O, Lin N, Snyder-Cappione JE. Aging, immune senescence, and immunotherapy:A comprehensive review. Seminars in oncology 2018;45(4):187-200 doi 10.1053/j.seminoncol.2018.08.006.

11. Sharma P, Hu-Lieskovan S, Wargo JA, Ribas A. Primary, Adaptive, and Acquired Resistance to Cancer Immunotherapy. Cel/2017;168(4):707-23 doi 10.1016/j.cell.2017.01.017.

12. Oh J, Magnuson A, Benoist C, Pittet MJ, Weissleder R. Age-related tumor growth in mice is related to 
integrin alpha 4 in CD8+ T cells. JC/ insight 2018;3(21) doi 10.1172/jci.insight.122961.

13. Hanahan D, Weinberg RA. Hallmarks of cancer: the next generation. Cell 2011;144(5):646-74 doi 10.1016/j.cell.2011.02.013.

14. Lee-Chang C, Bodogai M, Moritoh K, Olkhanud PB, Chan AC, Croft M, et al. Accumulation of 4-1BBL+ $B$ cells in the elderly induces the generation of granzyme-B+CD8+ T cells with potential antitumor activity. Blood 2014;124(9):1450-9 doi 10.1182/blood-2014-03-563940.

15. Zhang L, Zhang Z. Recharacterizing Tumor-Infiltrating Lymphocytes by Single-Cell RNA Sequencing. Cancer immunology research 2019;7(7):1040-6 doi 10.1158/2326-6066.CIR-18-0658.

16. Shalek AK, Satija R, Adiconis X, Gertner RS, Gaublomme JT, Raychowdhury R, et al. Single-cell transcriptomics reveals bimodality in expression and splicing in immune cells. Nature 2013;498(7453):236-40 doi 10.1038/nature12172.

17. Guo X, Zhang Y, Zheng L, Zheng C, Song J, Zhang Q, et al. Global characterization of T cells in non-small-cell lung cancer by single-cell sequencing. Nat Med 2018;24(7):978-85 doi 10.1038/s41591-018-0045-3.

18. Savas P, Virassamy B, Ye C, Salim A, Mintoff CP, Caramia F, et al. Single-cell profiling of breast cancer T cells reveals a tissue-resident memory subset associated with improved prognosis. Nat Med 2018;24(7):986-93 doi 10.1038/s41591-018-0078-7.

19. Zhang L, Yu X, Zheng L, Zhang Y, Li Y, Fang Q, et al. Lineage tracking reveals dynamic relationships of T cells in colorectal cancer. Nature 2018;564(7735):268-72 doi 10.1038/s41586-018-0694-x.

20. Helmink BA, Reddy SM, Gao J, Zhang S, Basar R, Thakur R, et al. B cells and tertiary lymphoid structures promote immunotherapy response. Nature 2020;577(7791):549-55 doi 10.1038/s41586-019-1922-8. 
21. Sun $Y$, Wu L, Zhong $Y$, Zhou K, Hou $Y$, Wang Z, et al. Single-cell landscape of the ecosystem in early-relapse hepatocellular carcinoma. Cel/2020 doi 10.1016/j.cell.2020.11.041.

22. Zheng C, Zheng L, Yoo JK, Guo H, Zhang Y, Guo X, et al. Landscape of Infiltrating T Cells in Liver Cancer Revealed by Single-Cell Sequencing. Cell 2017;169(7):1342-56 e16 doi 10.1016/j.cell.2017.05.035.

23. Stuart T, Butler A, Hoffman P, Hafemeister C, Papalexi E, Mauck WM, 3rd, et al. Comprehensive Integration of Single-Cell Data. Cel/2019;177(7):1888-902 e21 doi 10.1016/j.cell.2019.05.031.

24. Aran D, Looney AP, Liu L, Wu E, Fong V, Hsu A, et al. Reference-based analysis of lung single-cell sequencing reveals a transitional profibrotic macrophage. Nature immunology 2019;20(2):163-72 doi 10.1038/s41590-018-0276-y.

25. Qiu X, Hill A, Packer J, Lin D, Ma YA, Trapnell C. Single-cell mRNA quantification and differential analysis with Census. Nat Methods 2017;14(3):309-15 doi 10.1038/nmeth.4150.

26. Hanzelmann S, Castelo R, Guinney J. GSVA: gene set variation analysis for microarray and RNA-seq data. BMC Bioinformatics $2013 ; 14: 7$ doi 10.1186/1471-2105-14-7.

27. Tsuda T, Kim YT, Siskind GW, DeBlasio A, Schwab R, Ershler W, et al. Role of the thymus and T-cells in slow growth of B16 melanoma in old mice. Cancer research 1987;47(12):3097-100.

28. Carmona SJ, Siddiqui I, Bilous M, Held W, Gfeller D. Deciphering the transcriptomic landscape of tumor-infiltrating CD8 lymphocytes in B16 melanoma tumors with single-cell RNA-Seq. Oncoimmunology 2020;9(1) doi 10.1080/2162402x.2020.1737369.

29. Qu Y, Wen J, Thomas G, Yang W, Prior W, He W, et al. Baseline Frequency of Inflammatory Cxc19-Expressing Tumor-Associated Macrophages Predicts Response to Avelumab Treatment. Cell Rep 2020;32(1):107873 doi 10.1016/j.celrep.2020.107873. 
30. Arlauckas SP, Garren SB, Garris CS, Kohler RH, Oh J, Pittet MJ, et al. Arg1 expression defines immunosuppressive subsets of tumor-associated macrophages. Theranostics 2018;8(21):5842-54 doi 10.7150/thno.26888.

31. DeNardo DG, Ruffell B. Macrophages as regulators of tumour immunity and immunotherapy. Nature reviews Immunology 2019;19(6):369-82 doi 10.1038/s41577-019-0127-6.

32. Wculek SK, Cueto FJ, Mujal AM, Melero I, Krummel MF, Sancho D. Dendritic cells in cancer immunology and immunotherapy. Nature reviews Immunology 2020;20(1):7-24 doi 10.1038/s41577-019-0210-z.

33. Alshetaiwi H, Pervolarakis N, Mclntyre LL, Ma D, Nguyen Q, Rath JA, et al. Defining the emergence of myeloid-derived suppressor cells in breast cancer using single-cell transcriptomics. Sci Immunol 2020;5(44) doi 10.1126/sciimmunol.aay6017.

34. Wynn TA, Chawla A, Pollard JW. Macrophage biology in development, homeostasis and disease. Nature 2013;496(7446):445-55 doi 10.1038/nature12034.

35. Mantovani A, Sica A, Sozzani S, Allavena P, Vecchi A, Locati M. The chemokine system in diverse forms of macrophage activation and polarization. Trends in immunology 2004;25(12):677-86 doi 10.1016/j.it.2004.09.015.

36. Xuan W, Qu Q, Zheng B, Xiong S, Fan GH. The chemotaxis of M1 and M2 macrophages is regulated by different chemokines. Journal of leukocyte biology 2015;97(1):61-9 doi 10.1189/jlb.1A0314-170R.

37. Wu J, Li K, Peng W, Li H, Li Q, Wang X, et al. Autoinducer-2 of Fusobacterium nucleatum promotes macrophage M1 polarization via TNFSF9/LL-1 beta signaling. International immunopharmacology 2019;74:105724 doi 10.1016/j.intimp.2019.105724.

38. House IG, Savas P, Lai J, Chen AXY, Oliver AJ, Teo ZL, et al. Macrophage-Derived CXCL9 and 
CXCL10 Are Required for Antitumor Immune Responses Following Immune Checkpoint Blockade. Clinical cancer research : an official journal of the American Association for Cancer Research 2020;26(2):487-504 doi 10.1158/1078-0432.CCR-19-1868.

39. Sano M, Takahashi R, ljichi H, Ishigaki K, Yamada T, Miyabayashi K, et al. Blocking VCAM-1 inhibits pancreatic tumour progression and cancer-associated thrombosis/thromboembolism. Gut 2020 doi 10.1136/gutjnl-2020-320608.

40. De Palma M. Origins of Brain Tumor Macrophages. Cancer cell 2016;30(6):832-3 doi 10.1016/j.ccell.2016.11.015.

41. Mildner A, Jung S. Development and function of dendritic cell subsets. Immunity 2014;40(5):642-56 doi 10.1016/j.immuni.2014.04.016.

42. Jones EL, Wee JL, Demaria MC, Blakeley J, Ho PK, Vega-Ramos J, et al. Dendritic Cell Migration and Antigen Presentation Are Coordinated by the Opposing Functions of the Tetraspanins CD82 and CD37. Journal of immunology (Baltimore, Md : 1950) 2016;196(3):978-87 doi 10.4049/jimmunol. 1500357.

43. Joyce JA, Fearon DT. T cell exclusion, immune privilege, and the tumor microenvironment. Science (New York, NY) 2015;348(6230):74-80 doi 10.1126/science.aaa6204.

44. Konkel JE, Zhang D, Zanvit P, Chia C, Zangarle-Murray T, Jin W, et al. Transforming Growth Factor-beta Signaling in Regulatory T Cells Controls T Helper-17 Cells and Tissue-Specific Immune Responses. Immunity 2017;46(4):660-74 doi 10.1016/j.immuni.2017.03.015.

45. Koch S, Larbi A, Derhovanessian E, Ozcelik D, Naumova E, Pawelec G. Multiparameter flow cytometric analysis of CD4 and CD8 T cell subsets in young and old people. Immunity \& ageing : / \& $A$ 2008;5:6 doi 10.1186/1742-4933-5-6.

46. Bunztman A, Vincent BG, Krovi H, Steele S, Frelinger JA. The LCMV gp33-specific memory T cell 
repertoire narrows with age. Immunity \& ageing : / \& A 2012;9(1):17 doi 10.1186/1742-4933-9-17.

47. Beheshti A, Benzekry S, McDonald JT, Ma L, Peluso M, Hahnfeldt P, et al. Host age is a systemic regulator of gene expression impacting cancer progression. Cancer research 2015;75(6):1134-43 doi 10.1158/0008-5472.CAN-14-1053.

48. Mirza N, Pollock K, Hoelzinger DB, Dominguez AL, Lustgarten J. Comparative kinetic analyses of gene profiles of naive CD4+ and CD8+ T cells from young and old animals reveal novel age-related alterations. Aging ce//2011;10(5):853-67 doi 10.1111/j.1474-9726.2011.00730.x.

49. Quinn KM, Fox A, Harland KL, Russ BE, Li J, Nguyen THO, et al. Age-Related Decline in Primary CD8(+) T Cell Responses Is Associated with the Development of Senescence in Virtual Memory CD8(+) T Cells. Cell Rep2018;23(12):3512-24 doi 10.1016/j.celrep.2018.05.057.

50. Hurez V, Daniel BJ, Sun L, Liu AJ, Ludwig SM, Kious MJ, et al. Mitigating age-related immune dysfunction heightens the efficacy of tumor immunotherapy in aged mice. Cancer research 2012;72(8):2089-99 doi 10.1158/0008-5472.Can-11-3019.

51. Dutta S, Sengupta P. Men and mice: Relating their ages. Life Sci 2016;152:244-8 doi 10.1016/j.Ifs.2015.10.025.

52. Demetrius L. Aging in mouse and human systems: a comparative study. Ann $N Y$ Acad Sci 2006;1067:66-82 doi 10.1196/annals.1354.010.

53. Li H, van der Leun AM, Yofe I, Lubling Y, Gelbard-Solodkin D, van Akkooi ACJ, et al. Dysfunctional CD8 T Cells Form a Proliferative, Dynamically Regulated Compartment within Human Melanoma. Cell 2019;176(4):775-89 e18 doi 10.1016/j.cell.2018.11.043.

54. Garris CS, Arlauckas SP, Kohler RH, Trefny MP, Garren S, Piot C, et al. Successful Anti-PD-1 Cancer Immunotherapy Requires T Cell-Dendritic Cell Crosstalk Involving the Cytokines IFN-gamma and 
IL-12. Immunity2018;49(6):1148-61 e7 doi 10.1016/j.immuni.2018.09.024.

55. Saule P, Trauet J, Dutriez V, Lekeux V, Dessaint JP, Labalette M. Accumulation of memory T cells from childhood to old age: central and effector memory cells in CD4(+) versus effector memory and terminally differentiated memory cells in CD8(+) compartment. Mechanisms of ageing and development 2006;127(3):274-81 doi 10.1016/j.mad.2005.11.001.

56. Azizi E, Carr AJ, Plitas G, Cornish AE, Konopacki C, Prabhakaran S, et al. Single-Cell Map of Diverse Immune Phenotypes in the Breast Tumor Microenvironment. Cell 2018;174(5):1293-308 e36 doi 10.1016/j.cell.2018.05.060.

57. Manser AR, Uhrberg M. Age-related changes in natural killer cell repertoires: impact on NK cell function and immune surveillance. Cancer immunology, immunotherapy : C// 2016;65(4):417-26 doi 10.1007/s00262-015-1750-0.

58. Lutz CT, Karapetyan A, Al-Attar A, Shelton BJ, Holt KJ, Tucker JH, et al. Human NK cells proliferate and die in vivo more rapidly than $\mathrm{T}$ cells in healthy young and elderly adults. Journal of immunology (Baltimore, Md : 1950) 2011;186(8):4590-8 doi 10.4049/jimmunol.1002732.

59. Chester C, Sanmamed MF, Wang J, Melero I. Immunotherapy targeting 4-1BB: mechanistic rationale, clinical results, and future strategies. Blood 2018;131(1):49-57 doi 10.1182/blood-2017-06-741041.

60. Shahaf G, Johnson K, Mehr R. B cell development in aging mice: lessons from mathematical modeling. Int Immuno/ 2006;18(1):31-9 doi 10.1093/intimm/dxh346.

61. Ademokun A, Wu YC, Dunn-Walters D. The ageing B cell population: composition and function. Biogerontology 2010;11(2):125-37 doi 10.1007/s10522-009-9256-9.

62. Miller JP, Cancro MP. B cells and aging: balancing the homeostatic equation. Experimental gerontology 2007;42(5):396-9 doi 10.1016/j.exger.2007.01.010. 
63. Buffa S, Pellicano M, Bulati M, Martorana A, Goldeck D, Caruso C, et al. A novel B cell population revealed by a CD38/CD24 gating strategy: CD38(-)CD24 (-) B cells in centenarian offspring and elderly people. Age (Dordr) 2013;35(5):2009-24 doi 10.1007/s11357-012-9488-5.

64. Schlitzer A, McGovern N, Ginhoux F. Dendritic cells and monocyte-derived cells: Two complementary and integrated functional systems. Semin Cell Dev Biol 2015;41:9-22 doi 10.1016/j.semcdb.2015.03.011.

65. Bottcher JP, Bonavita E, Chakravarty P, Blees H, Cabeza-Cabrerizo M, Sammicheli S, et al. NK Cells Stimulate Recruitment of cDC1 into the Tumor Microenvironment Promoting Cancer Immune Control. Cell2018;172(5):1022-37 e14 doi 10.1016/j.cell.2018.01.004.

66. Binnewies M, Mujal AM, Pollack JL, Combes AJ, Hardison EA, Barry KC, et al. Unleashing Type-2 Dendritic Cells to Drive Protective Antitumor CD4(+) T Cell Immunity. Cell2019;177(3):556-71 e16 doi 10.1016/j.cell.2019.02.005.

67. Watanabe S, Alexander M, Misharin AV, Budinger GRS. The role of macrophages in the resolution of inflammation. The Journal of clinical investigation 2019;129(7):2619-28 doi 10.1172/JCI124615.

68. Canton J. Macropinocytosis: New Insights Into Its Underappreciated Role in Innate Immune Cell Surveillance. Frontiers in immunology 2018;9:2286 doi 10.3389/fimmu.2018.02286.

69. Lorenzo-Sanz L, Munoz P. Tumor-Infiltrating Immunosuppressive Cells in Cancer-Cell Plasticity, Tumor Progression and Therapy Response. Cancer Microenviron 2019;12(2-3):119-32 doi 10.1007/s12307-019-00232-2.

70. Liu Y, Cao X. Immunosuppressive cells in tumor immune escape and metastasis. J Mol Med (Berl) 2016;94(5):509-22 doi 10.1007/s00109-015-1376-x.

71. Tsaknaridis L, Spencer L, Culbertson N, Hicks K, LaTocha D, Chou YK, et al. Functional assay for 
human CD4+CD25+ Treg cells reveals an age-dependent loss of suppressive activity. J Neurosci Res 2003;74(2):296-308 doi 10.1002/jnr. 10766.

72. Hwang KA, Kim HR, Kang I. Aging and human CD4(+) regulatory T cells. Mechanisms of ageing and development 2009;130(8):509-17 doi 10.1016/j.mad.2009.06.003.

73. Gabrilovich DI, Nagaraj S. Myeloid-derived suppressor cells as regulators of the immune system. Nature reviews Immunology 2009;9(3):162-74 doi 10.1038/nri2506.

74. Motallebnezhad M, Jadidi-Niaragh F, Qamsari ES, Bagheri S, Gharibi T, Yousefi M. The immunobiology of myeloid-derived suppressor cells in cancer. Tumour Bio/ 2016;37(2):1387-406 doi 10. 1007/s13277-015-4477-9.

75. Shields CWt, Evans MA, Wang LL, Baugh N, lyer S, Wu D, et al. Cellular backpacks for macrophage immunotherapy. Sci Adv2020;6(18):eaaz6579 doi 10.1126/sciadv.aaz6579.

76. Aspord C, Leccia MT, Charles J, Plumas J. Plasmacytoid dendritic cells support melanoma progression by promoting Th2 and regulatory immunity through OX40L and ICOSL. Cancer immunology research 2013;1(6):402-15 doi 10.1158/2326-6066. CIR-13-0114-T.

\section{Figure legend}

Figure 1. Changes in immune cell composition of the TME during aging (A) Tumor growth of B16 melanoma in young $(n=3)$ and old $(n=3)$ mouse models. Mice with the tumor size of $\left(\right.$ length $\times$ width $\left.{ }^{2}\right) / 2 \mathrm{~mm}^{2}$ were monitored.

(B) Schematic diagram of the experimental design, single-cll RNA sequencing, data analysis, and validation.

(C) Heatmap showing the relative expression (expression of normalized log2 (count +1$)$ ) of 
marker genes across different immune cell types.

(D) tSNE projections of immune cells in tumors of young (upper) and old (below) mice.

(E) The proportions of various immune cell types in tumors of young and old mice.

cDC: conventional dendritic cells; pDC: plasmacytoid dendritic cells; NK: natural killing cells;

Figure 2. Myeloid cell composition of the TME in young and old mice

(A) Pie charts showing the proportions of four major myeloid cell types in tumors of young and old mice.

(B) Heatmap showing the relative expression (expression of normalized log2 (count +1$)$ ) of top differentially expressed genes across different myeloid cell clusters.

(C) UMAP projections of myeloid cell subpopulations in tumors of young (upper) and old (below) mice.

(D) The proportions of various myeloid cell clusters in young and old mice.

Figure 3. Macrophage cell subtypes and their heterogeneity in tumors of young and old mice

(A) Heatmap showing the relative expression of marker genes across various macrophage subsets.

(B) Comparison of the proportions of five macrophage subsets in young and old mice.

(C) Expression profiles of chemokines in different macrophage subsets.

(D) Bubble plots showing the scores (represented by the color gradient) of different gene sets and proportions (represented by the size of bubble) of each macrophage cluster in 
young and old mice. The gene set score is calculated by averaging the z-scores of gene expression values of all genes in this gene set.

The gene expression in A \& C is represented as expression of normalized log2 (count +1$))$. MC1 - MC5: five clusters of macrophages.

Figure 4. Dendritic cell subtypes and their heterogeneity in tumors of young and old mice (A) Violin plots comparing the expression levels of representative marker genes among different DC subtypes.

(B) Pie charts showing the proportions of four DC subtypes in tumors of young and old mice.

(C) Violin plots comparing the gene expression among different DC subtypes.

( $D$ and E) Percentages of $\mathrm{Lag}^{+} \mathrm{DC}$ in the $\mathrm{dLN}(\mathrm{D})$ and tumor tissues(E) of young and old $\operatorname{groups}(\mathrm{n}=3)$.

(F) Bubble plots showing the scores (represented by the color gradient) of different gene sets and proportions (represented by the size of bubble) of each DC cluster in young and old mice. The gene set score was calculated by averaging the z-scores of gene expression values of all genes in this gene set.

(G) Violin plot showing gene expression in MoDC in tumors of young and old mice The gene expression in A,C, and G is represented as expression of normalized log2 (count $+1)$.

Figure 5. T cell subtypes and their heterogeneity in tumors of young and old mice (A) UMAP projections of T cells in tumors of young (left) and old (right) mice. 
( $B$ and $C)$ Expression of signature genes $(B)$ and genes essential for $T$ cell function( $C$ ) projected onto UMAP plots in(A). Color scale shows z-score transformation of log2 (count $+1)$.

(D) Bar graph showing the percentages of various T cell subtypes in the tumors of young and old mice.

Figure 6. Diversity of tumor-infiltrating $\mathrm{CD}^{+} \mathrm{T}$ lymphocytes and their functional states in young and old mice

(A) Pie charts showing the proportions of $\mathrm{CD} 8^{+} \mathrm{T}$ subtypes in young and old mice.

(B) Percentages of PD $-1^{+} \mathrm{LAG}^{+} \mathrm{CD}^{+} \mathrm{T}$ (left) and Tim- $3^{+} \mathrm{LAG}^{+} \mathrm{CD} 8^{+} \mathrm{T}$ (right) cells in tumor tissue of young and old mice $(n=5)$.

(C) Percentages of IFN- $\gamma^{+} \mathrm{CD}^{+} \mathrm{T}$ (left) and GranzymeB ${ }^{+} \mathrm{CD} 8^{+} \mathrm{T}$ (right) cells in tumor tissue of young and old mice $(\mathrm{n}=3)$.

(D) tSNE plots representing T-cell receptor (TCR) profiles of top10 clonotypes in tumors of young (left) and old (right) mice.

$\left(\mathrm{E}\right.$ and $\mathrm{F}$ ) Differentiation trajectory of $\mathrm{CD}^{+} \mathrm{T}$ cells reconstructed by monocle2 using single-cell RNA-seq data. Color scale indicates either the ordering of cell in pseudotime $(E)$ or the cell state $(\mathrm{F})$.

(G) Venn plot showing the number of shared TCR clones between exhausted CD8+ T and other CD8+ T cells in tumors of young and old mice.

(H) Bar graph showing the percentages of TCR sequences shared by exhausted CD8+ T and other CD8+ $T$ cells in young and old mice. 
Figure 7. Characterization of tumor infiltrating naive and memory like $\mathrm{CD} 8^{+} \mathrm{T}$ cells in young and old mice

(A) Pie charts showing the proportions of four T cells subtypes in young and old mice.

( $B$ and $C$ ) Percentages of $C D 8^{+} E M \_l i k e ~ T(B)$ and $C D 4^{+} E M \_l i k e ~ T(C)$ in the dLN (upper) and tumor tissues (below) of young and old.

(D) Pathway enrichment result of top differential expressed genes in memory_like T cells.

(E) Violin plot showing the gene expression in naive_T and different memory_like T cells.

(F) Differentiation trajectory of $\mathrm{CD} 8^{+} \mathrm{EM} \_$like $\mathrm{T}$ cytotoxic_CD8 ${ }^{+} \mathrm{T}$ and exhausted_CD8 ${ }^{+} \mathrm{T}$ cells reconstructed by monocle2 using single-cell RNA-seq data from young and old mice. Colors indicate the cell differentiation states.

\section{Supplementary Figure legend}

Fig. S1. scRNA-seq profile of tumor-infiltrating immune cells in young and old mice

(A) PCA plot of 9981 high-quality immune cells showed no batch effect existing between young mice and old mice.

(B) tSNE visualization of the cell-cycle scores for different immune cells

(C) tSNE plot of the distribution of all immune cells, colored by age groups.

(D) tSNE plot of all immune cells from tumors of both young and old mice.

(E) Dot plot showing the expression levels of marker genes in different cell clusters. The expression was measured as the $\log _{2}$ (count+ 1$)$. 
Fig. S2. Characterization of myeloid cells using scRNA-seq data

(A) Scatter plot comparing the gene expression profiles of neutrophils in tumors of young and old mice. Each dot denoted an individual gene. The criteria for gene changes are $|\mathrm{FC}|>1$

(B) UMAP plot of macrophages, dendritic cells, and monocytes, colored by age groups.

(C) UMAP plot of macrophages, dendritic cells, and monocytes in tumors of both young and old mice.

(D and E) Dot plot showing the expression levels of representative DC markers(D) and macrophage markers(E) of in different cell clusters. The expression was measured as the $\log _{2}($ count +1$)$

Fig. S3. Characterization of tumor infiltrating macrophages

Violin plot comparing the expression levels of representative signatures among different macrophage subtypes.

(B and $\mathrm{C})$ Violin plot comparing the expression levels of representative $\mathrm{MC2}$ signatures(B) and MC4 signatures $(\mathrm{C})$ in young and old mice.

The gene expression was measured as the $\log _{2}($ count +1$)$. markers $(E)$ of in different cell clusters.

The gene expression was measured as the $\log _{2}($ count +1$)$.

Fig. S4. Characterization of tumor infiltrating dendritic cells

(A) Violin plot comparing the expression levels of functional genes among different dendritic 
cell subtypes.

(B) Violin plot showing the expression of Tnf-family genes in various dendritic cells.

(C) Dot plot showing the gene expression of $\mathrm{H} 2$ components in various dendritic cells.

The gene expression was measured as the $\log _{2}($ count +1$)$.

Fig. S5. Characterization of tumor infiltrating T cells

(A) Violin plot showing expression levels of representative marker genes for different T cells.

(B) Dot plot showing the expression levels of representative marker genes of different $\mathrm{T}$ cells.

(C) UMAP plot showing the distribution of different tumor infiltrating T cells, colored by the cell subtypes.

The gene expression was measured as the $\log _{2}(\operatorname{count}+1)$.

Fig. S6. Analysis of tumor infiltrating Tregs

(A) GSEA plots of the signature gene sets in tumor infiltrating Tregs of young mice vs. old mice. NES: Normalized Enrichment Score.

(B) Percentages of tumor-infiltrating $n$ Tregs $\left(\right.$ Helios $\left.^{+}\right)$in the young and old mice $(n=3)$.

(C) Histogram of Helios expression in young and old Tregs $(n=3)$.

(D) Violin plot showing expression levels of Tregs functional genes in young and old mice.

The expression was measured as the $\log _{2}($ count +1$)$.

( $E$ and $F$ ) Percentages of TGF- $\beta^{+}$Tregs in the $\mathrm{dLN}(\mathrm{E})$ and tumor tissues(F) of young and old $\operatorname{mice}(n=3)$. 
Error bar is mean \pm SEM. The significant level is determined by two-tailed, paired Student's t-test.

Fig. S7. Analysis of $\mathrm{CD} 8^{+} \mathrm{T}$ cell differentiation status

(A) Percentages of IFN- $\mathrm{y}^{+} \mathrm{CD} 8^{+} \mathrm{T}$ (left) and GranzymeB ${ }^{+} \mathrm{CD} 8^{+} \mathrm{T}$ (right) cells in $\mathrm{dLN}$ of young and old mice $(n=3)$.

(B) Cell differentiation trajectory of activated $\mathrm{CD}^{+} \mathrm{T}$ cells (right), cytotoxic $\mathrm{CD} 8^{+} \mathrm{T}$ cells(middle), and exhausted CD8 ${ }^{+} \mathrm{T}$ cells (left) reconstructed by Monocle2 using single-cell RNA-seq data. Color scale indicates the ordering of cells in pseudotime.

(C) Dot plot showing expression of genes associated with $\mathrm{T}$ cell states in cytotoxic $\mathrm{CD}^{+} \mathrm{T}$ cells(up) and exhausted CD8+ T cells (down).

(D and E) Gene Set Variation Analysis (GSVA) results using differential expressed genes in cytotoxic $C D 8^{+} T$ cells(D) and exhausted $C D 8^{+} T$ cells $(E)$.

(F) Bubble plots showing the scores (represented by the color gradient) of different gene sets and proportions (represented by the size of bubble) of each T cell groups in young and old mice. The gene set score was calculated by averaging the z-scores of gene expression values of all genes in this gene set.

Fig. S8. Analysis of effector memory like T cell differentiation status

( $A$ and $B$ ) Violin plot comparing the expression levels of representative signatures of $C D 8^{+}$

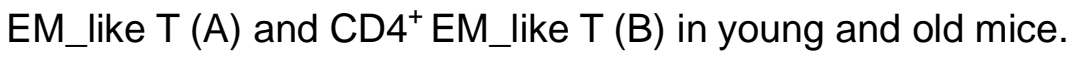

(C) Cell differentiation trajectory of CD8 ${ }^{+}$EM_like T, Cytotoxic CD8+ T and Exhausted CD8 ${ }^{+}$ 
T cells reconstructed by Monocle2 using single-cell RNA-seq data. Color scale indicates the ordering of cells in pseudotime.

(D) Cell differentiation trajectory of $\mathrm{CD}^{+} \mathrm{EM}$ _like T (left), cytotoxic CD8+ T(middle) and exhausted CD8+ T cells(right) reconstructed by Monocle2 using single-cell RNA-seq data, colored by cell states.

The gene expression in $A$ and $B$ was measured as the $\log _{2}(\operatorname{count}+1)$.

Fig. S9. Analysis of tumor-infiltrating NK cells

(A) GSEA plots of the signature gene sets in tumor infiltrating NK cells by comparing young with old mice. NES: Normalized Enrichment Score.

(B) Gene Set Variation Analysis (GSVA) identified enriched GO terms for NK cells in young vs. old mice.

(C) Violin plot showing expression levels of genes associated with NK cell's function in young and old mice.

The gene expression was measured as the $\log _{2}($ count +1$)$.

Fig. S10. Analysis of tumor infiltrating B cells

(A) GSEA plots of the signature gene sets in tumor infiltrating B cells by comparing young with old mice. NES: Normalized Enrichment Score.

(B) Proportions of tumor-infiltrating B cells in the young and old mice $(n=3)$.

(C) Violin plot showing expression levels of B cell marker genes in young and old mice. The gene expression was measured as the $\log _{2}($ count +1$)$. 
bioRxiv preprint doi: https://doi.org/10.1101/2021.02.23.432366; this version posted February 23, 2021. The copyright holder for this preprint (which was not certified by peer review) is the author/funder. All rights reserved. No reuse allowed without permission.

Error bar is mean \pm SEM. The significant level is determined by two-tailed, paired Student's t-test. 
Fig. 1

A

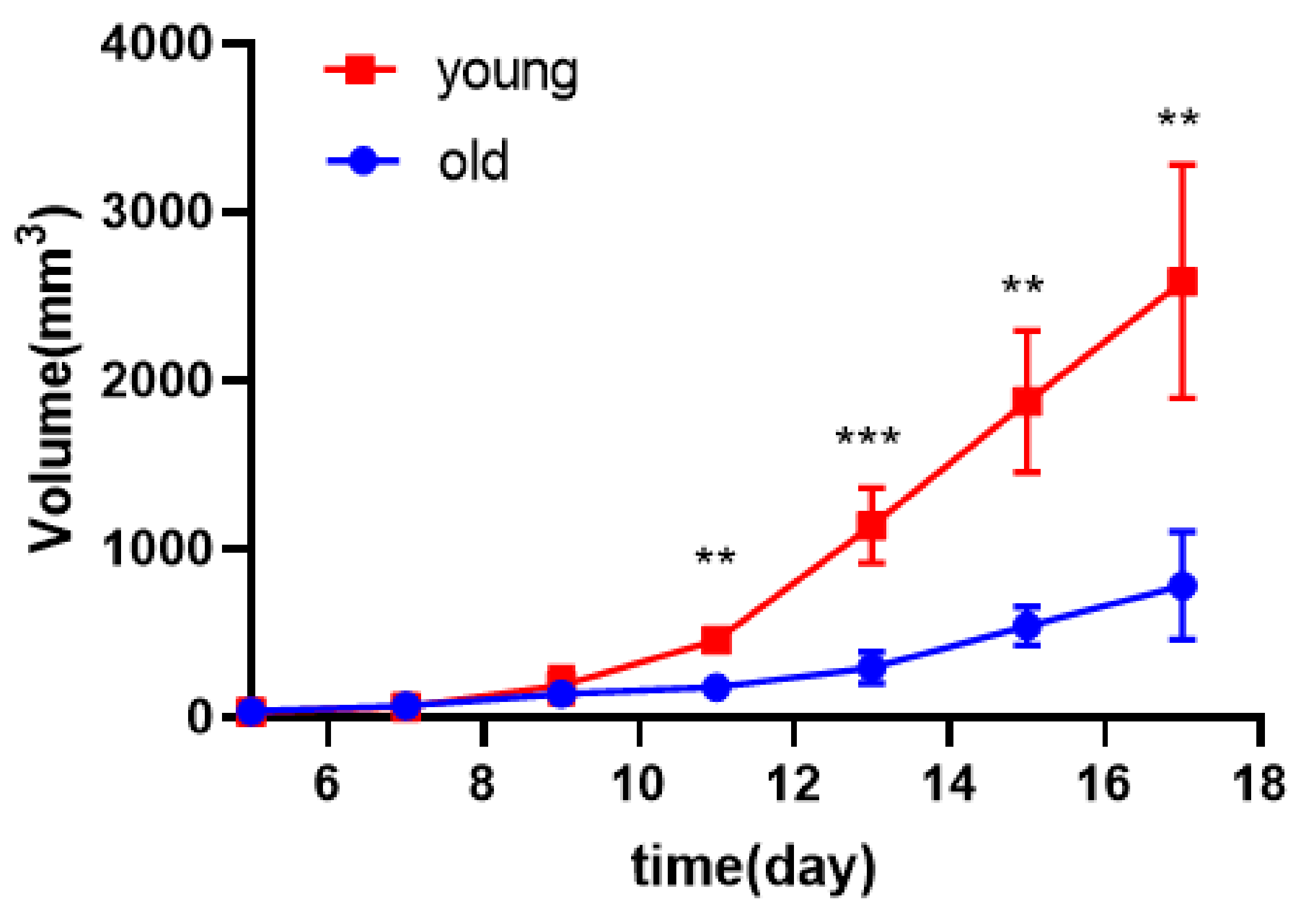

C

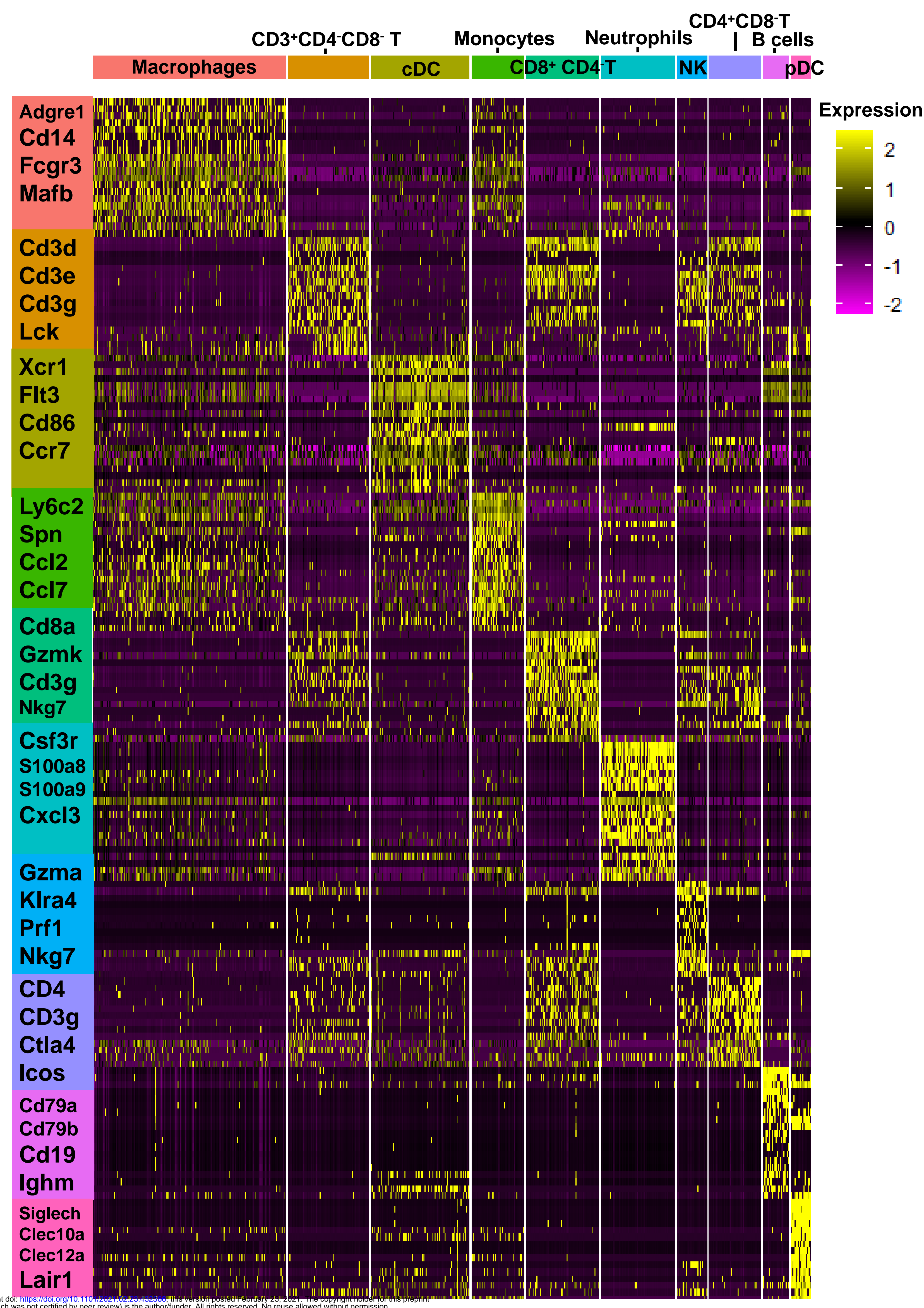

B

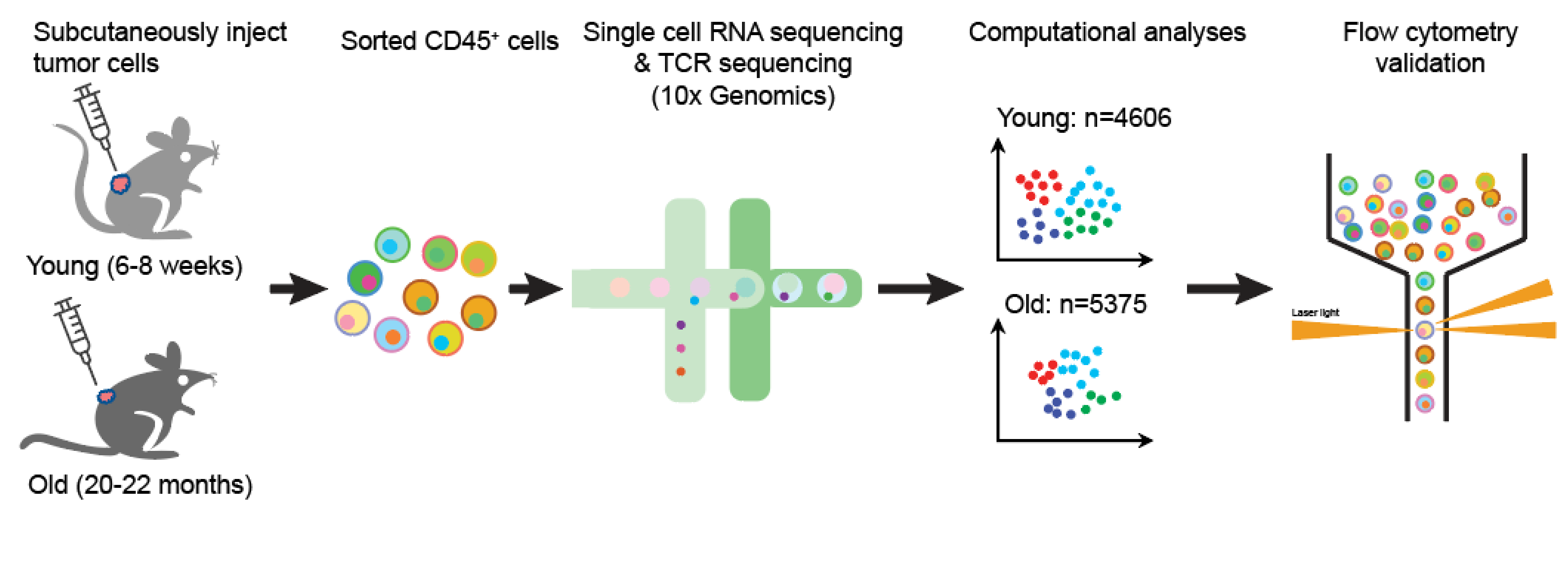

D

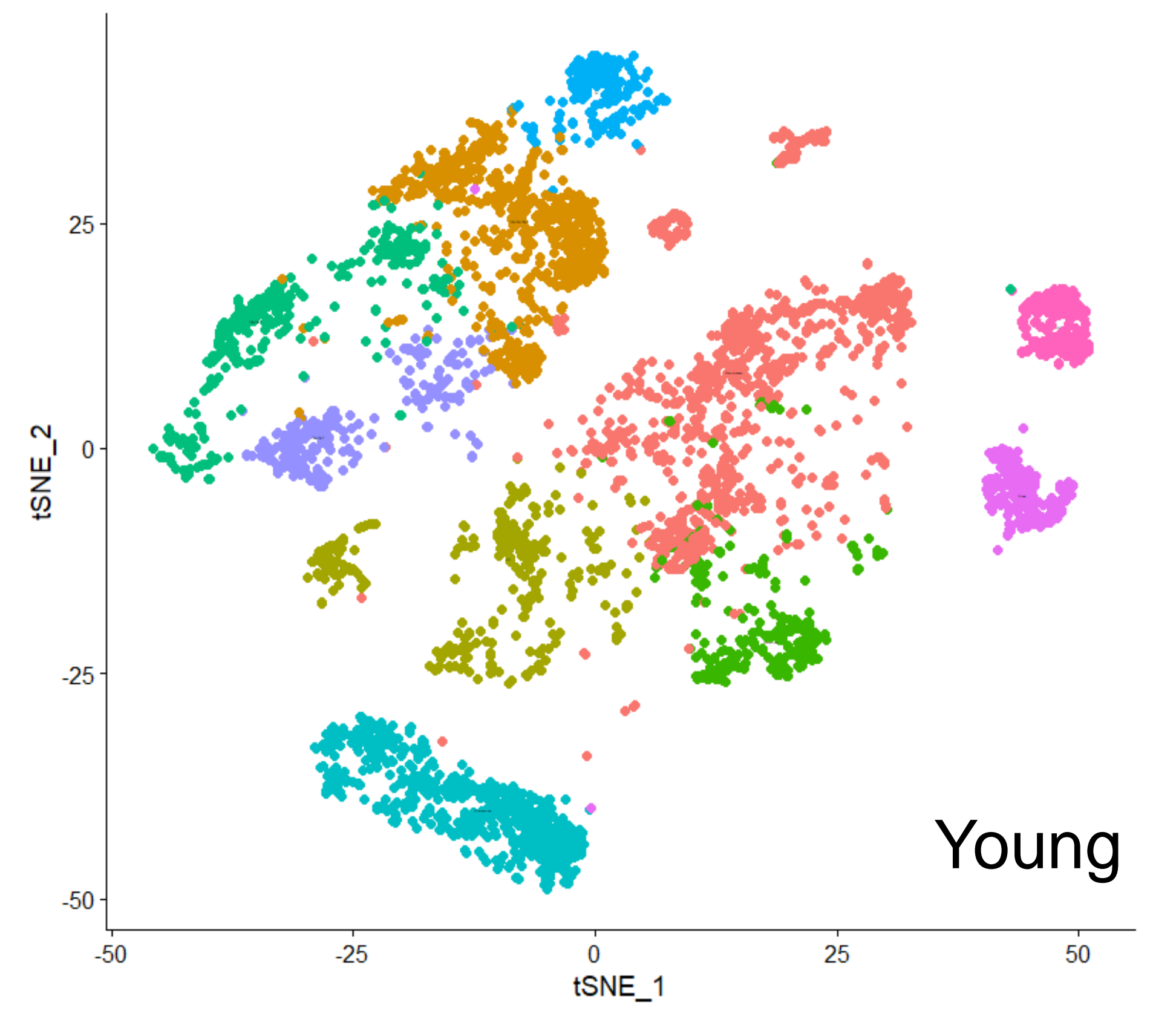

Monocytes

Macrophages

- CDC

pDC

Neutrophils

B cell

NK

CD3 ${ }^{+}$CD4 4 CD8-T

CD4 ${ }^{+C D 8}{ }^{-T}$

CD8+CD4-T

$\mathrm{E}$

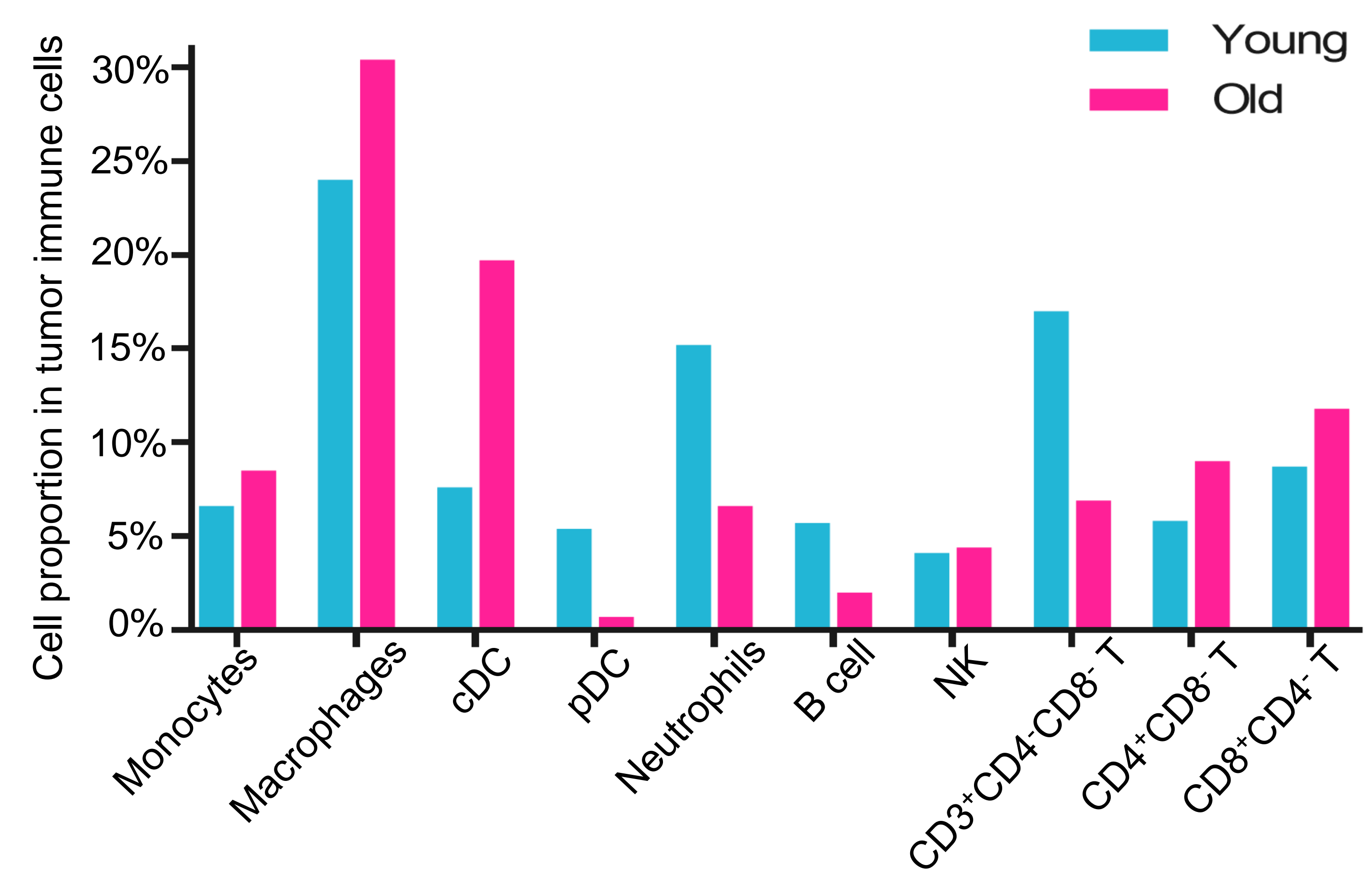


Fig. 2

A

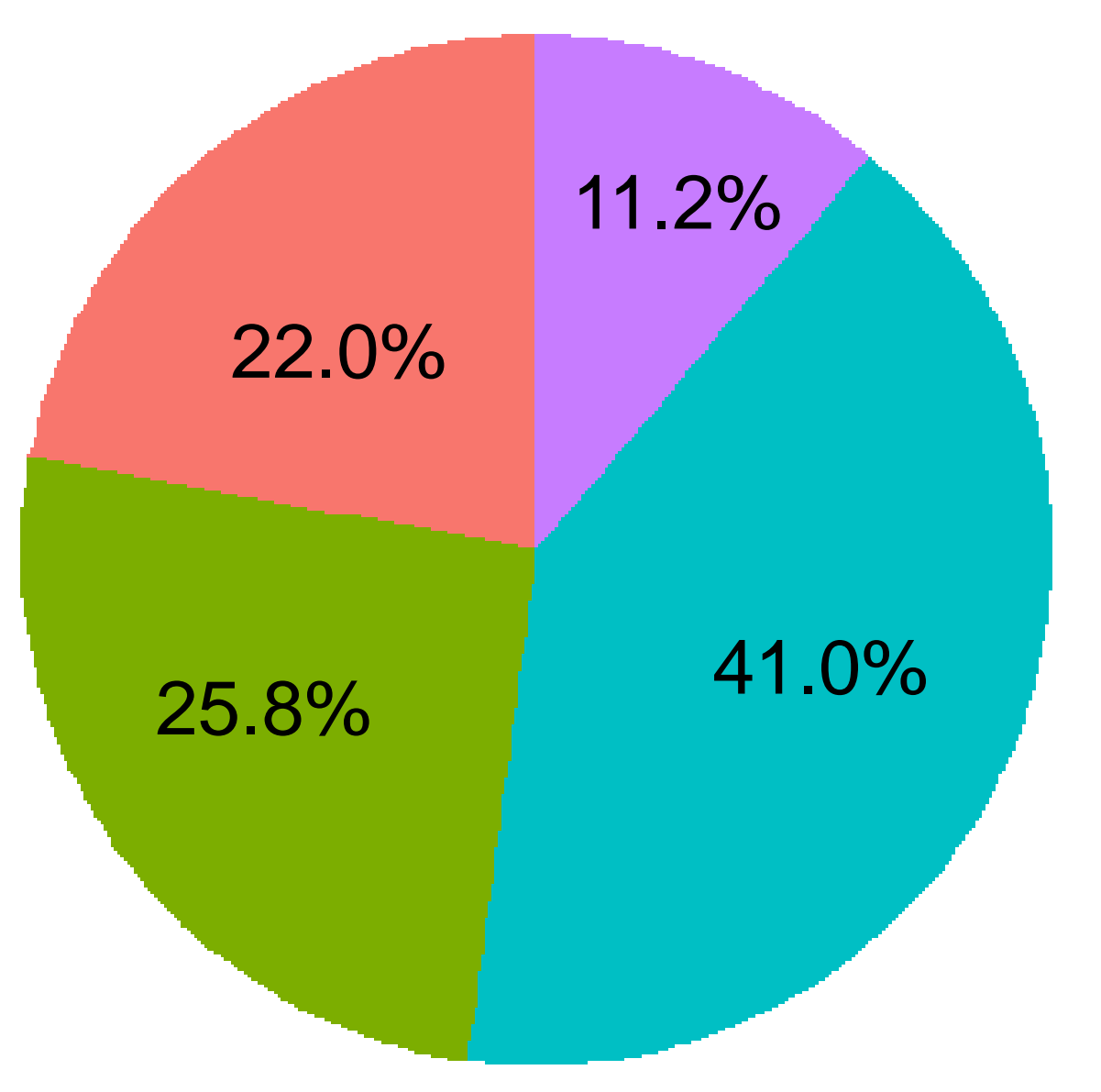

Young

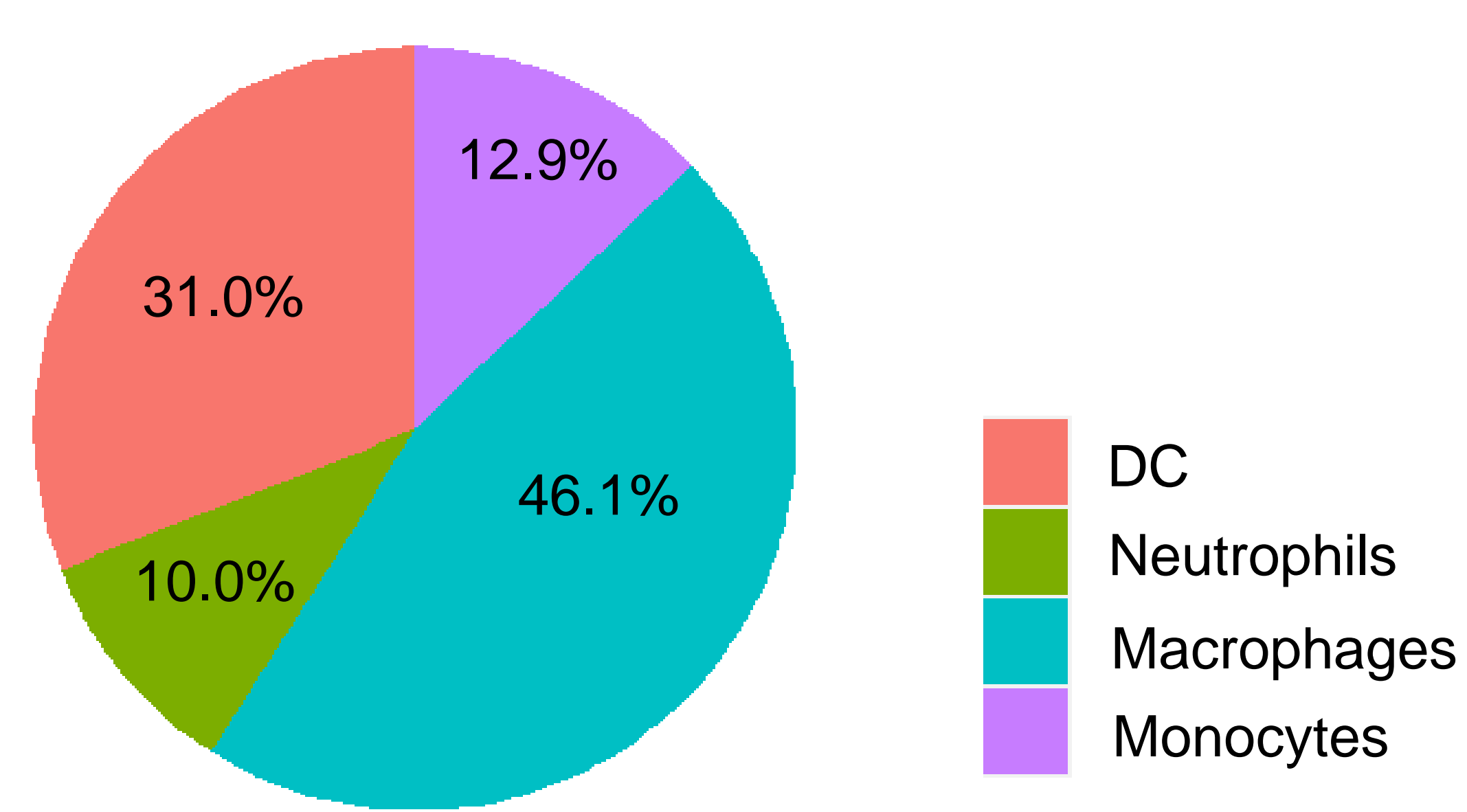

Old
B

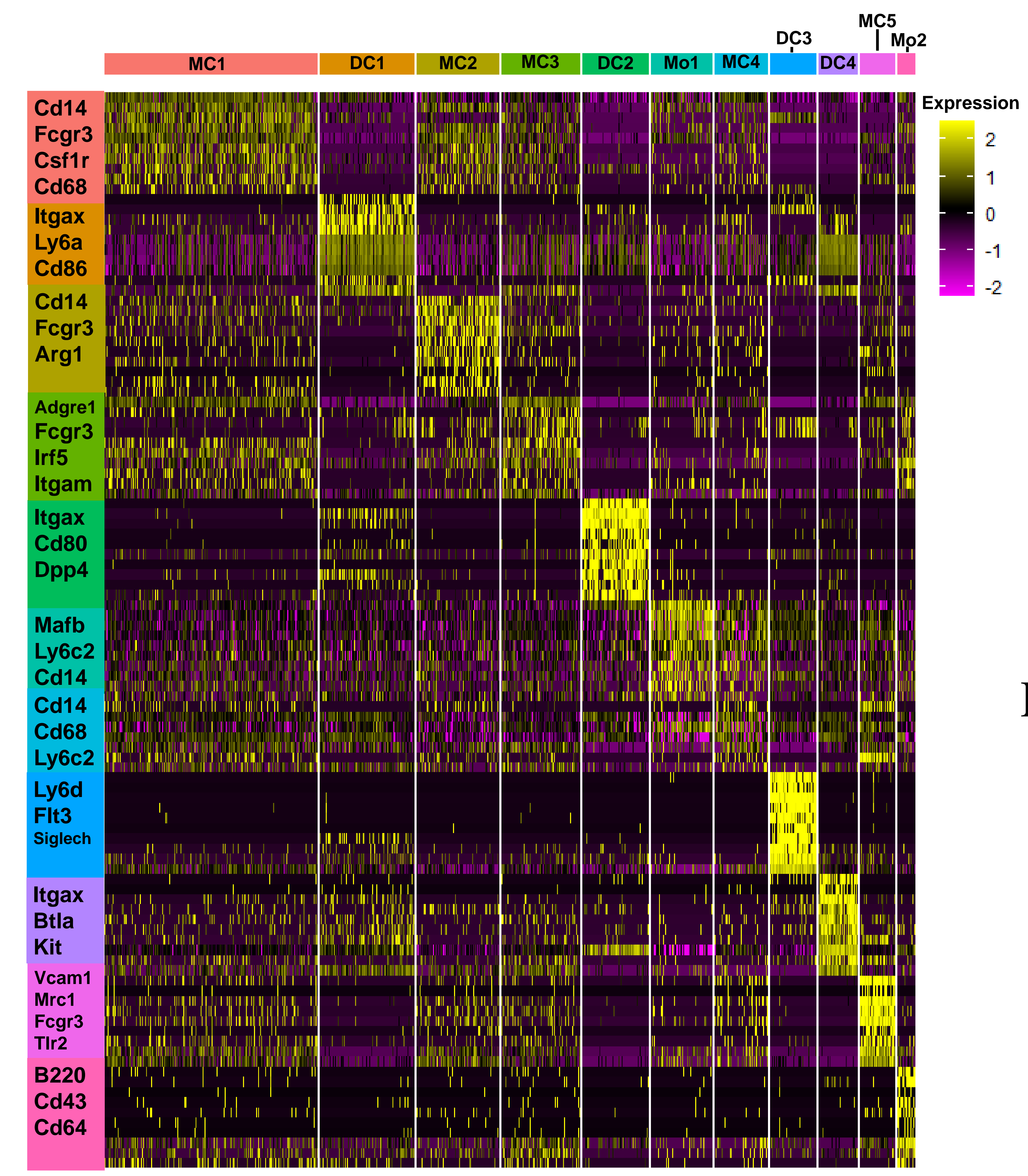

C

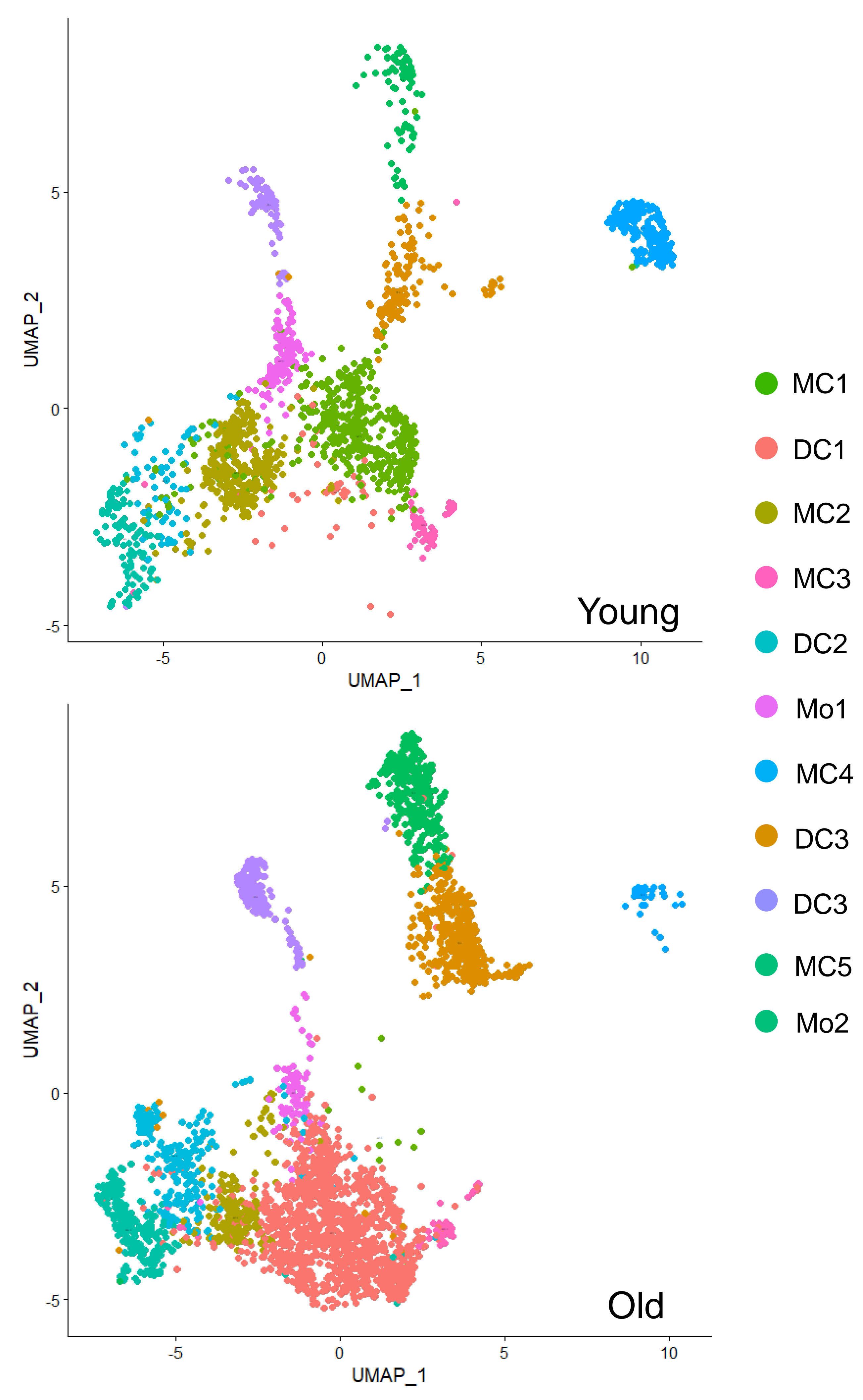

D

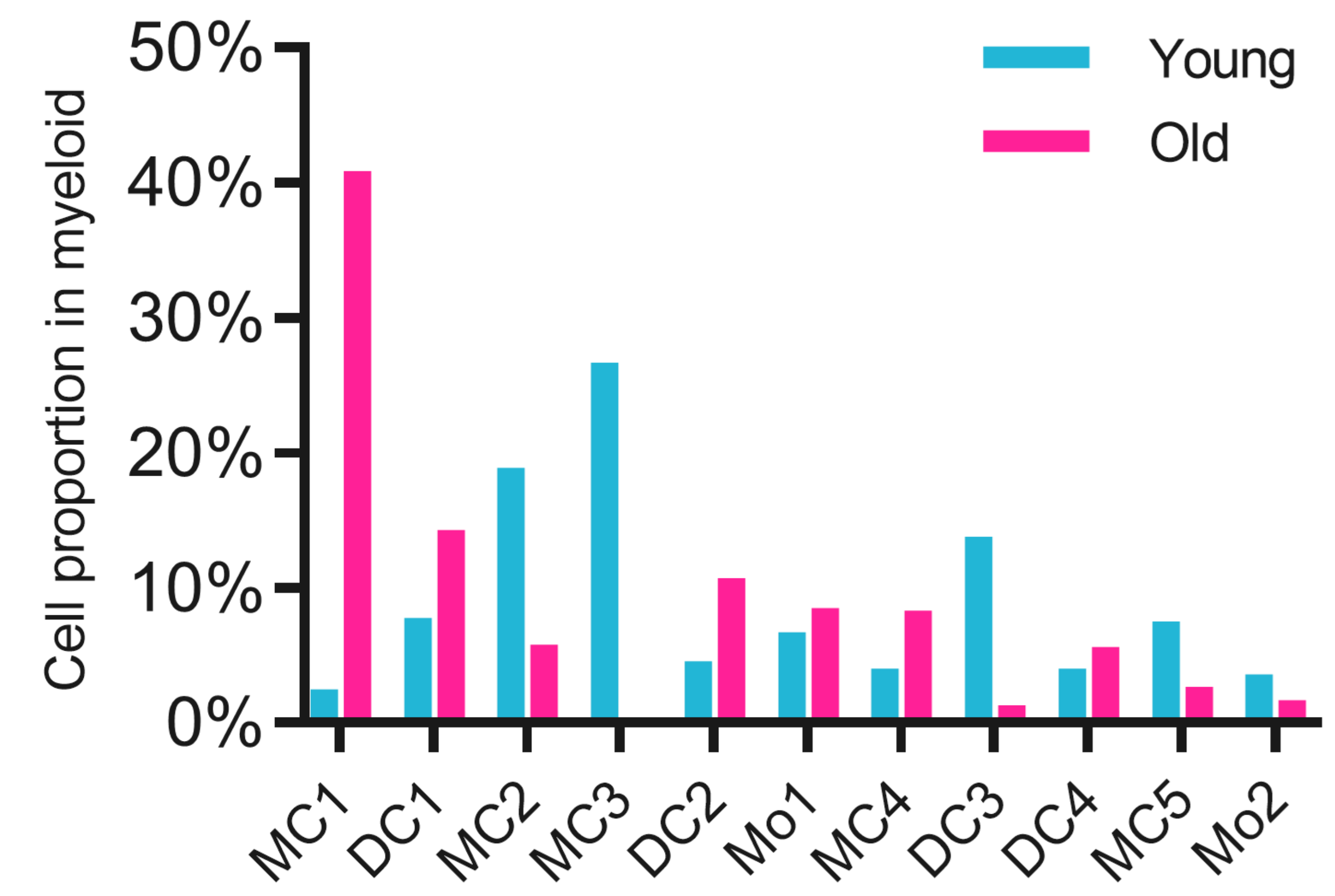


Fig. 3

A

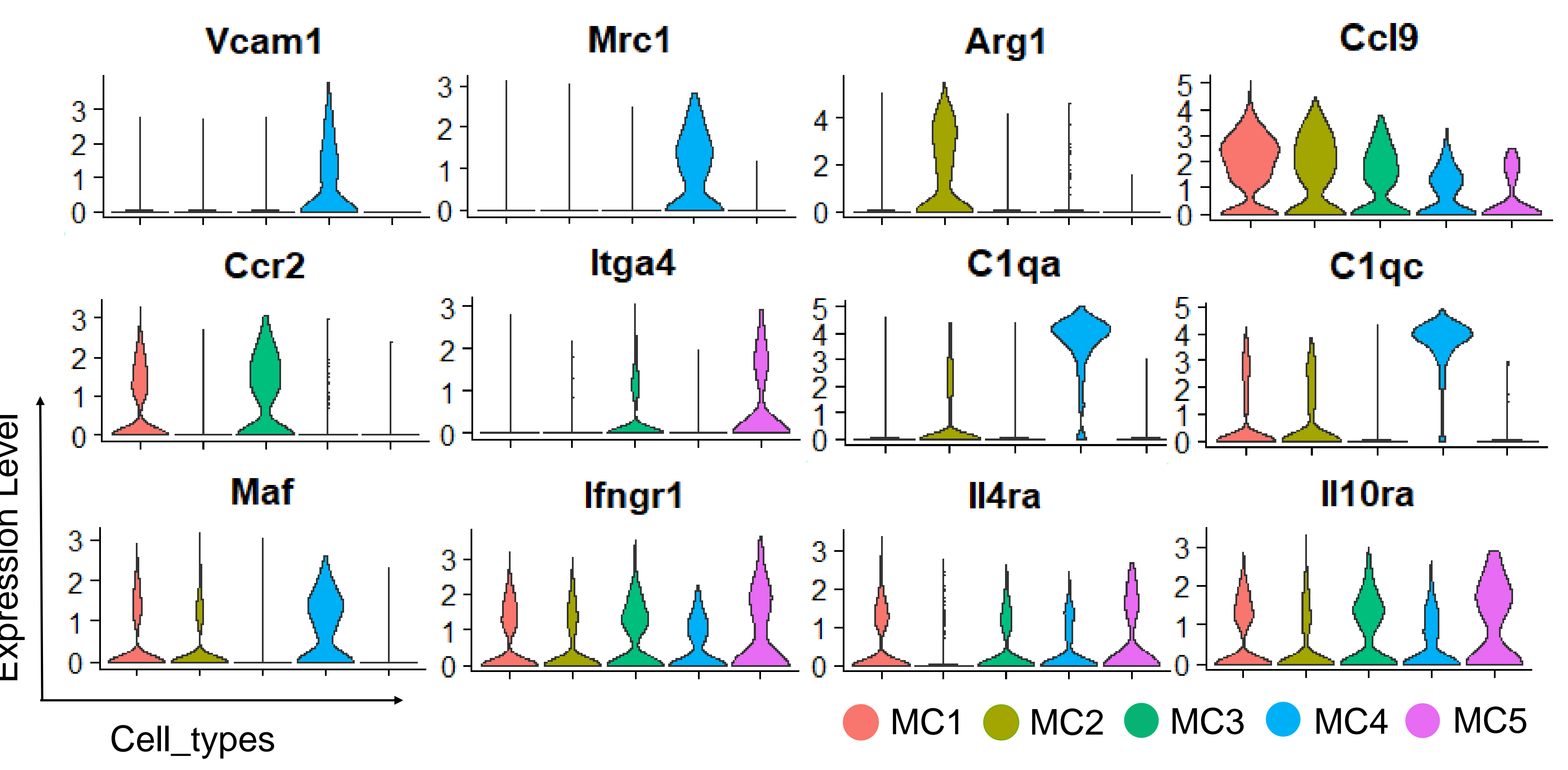

C

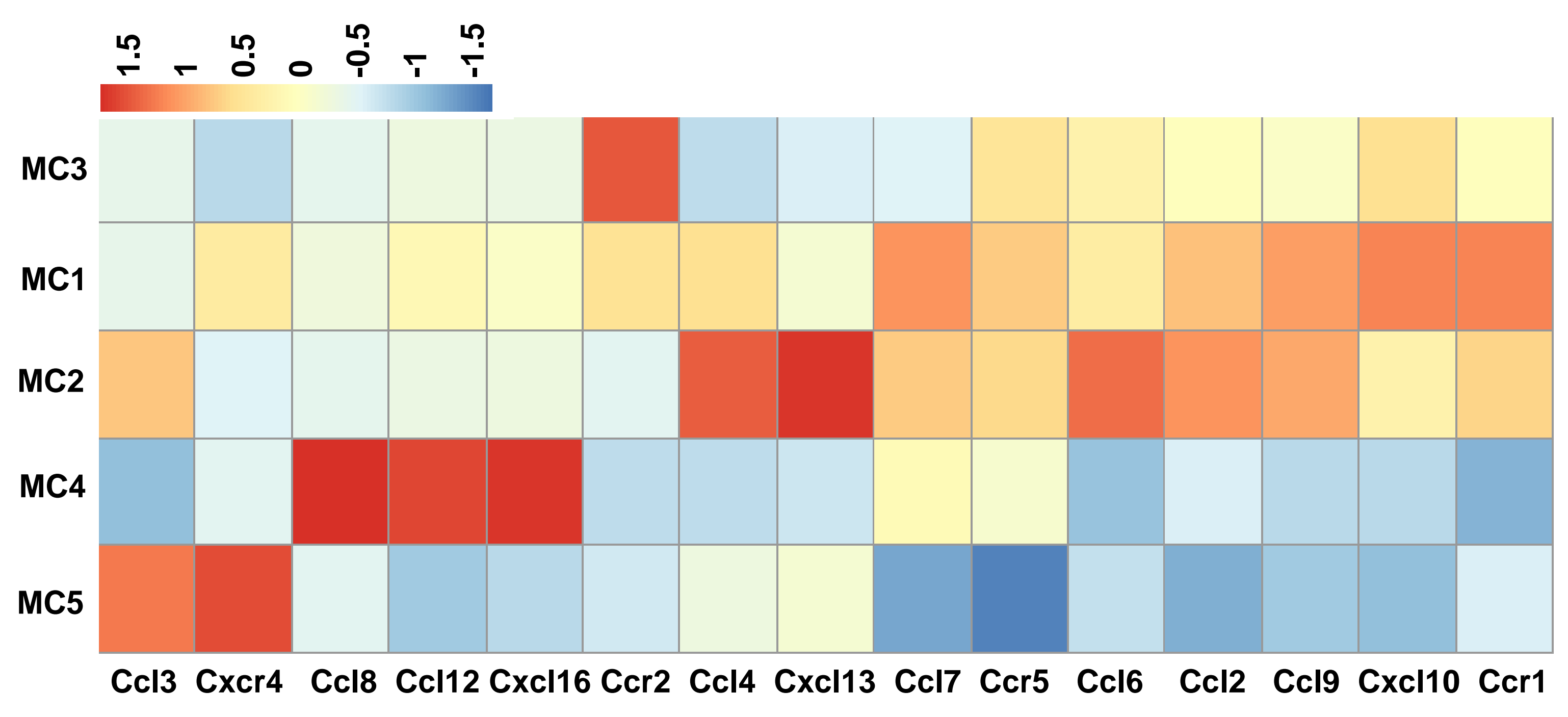

B

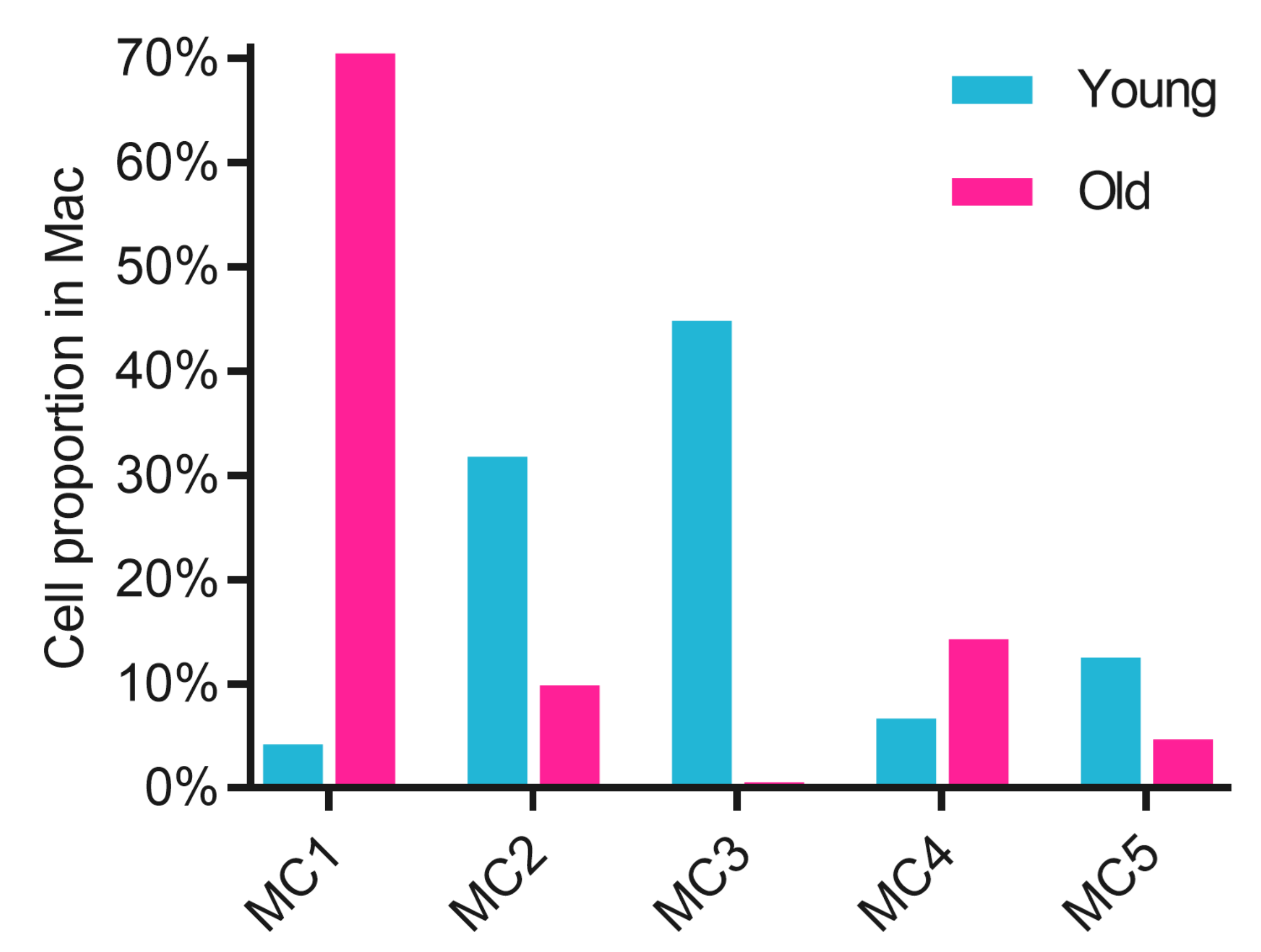

D

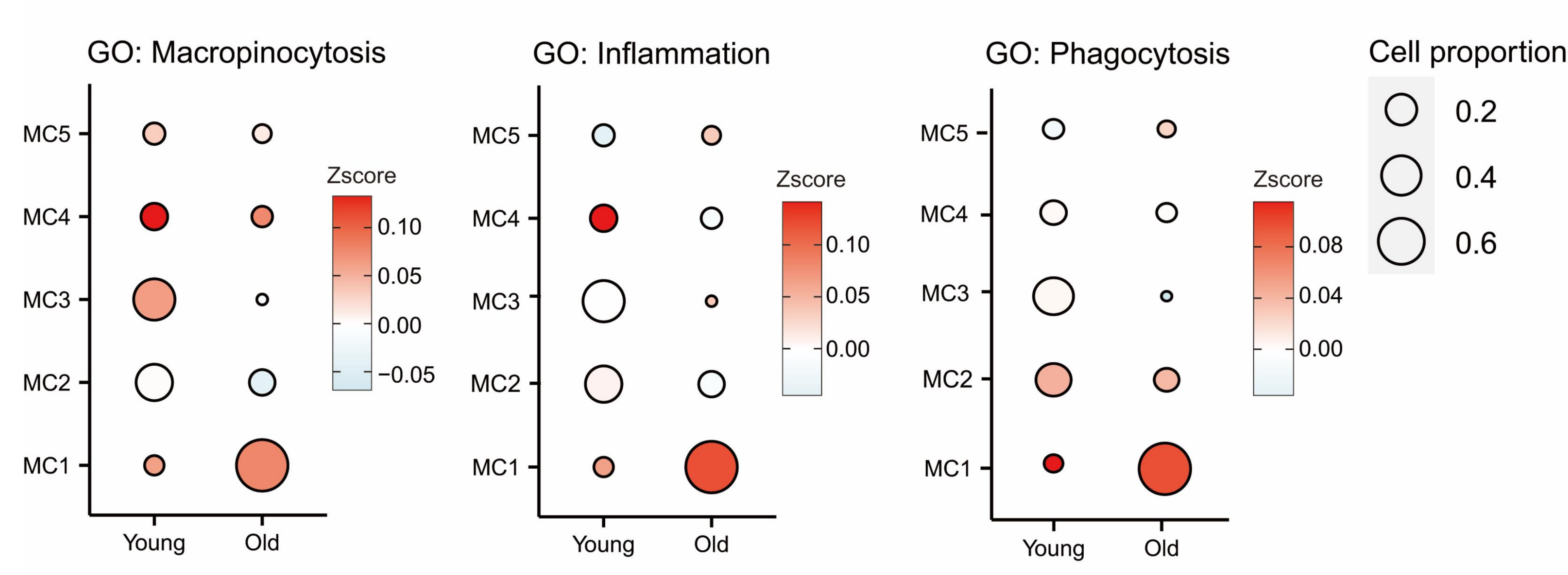


Fig. 4

A
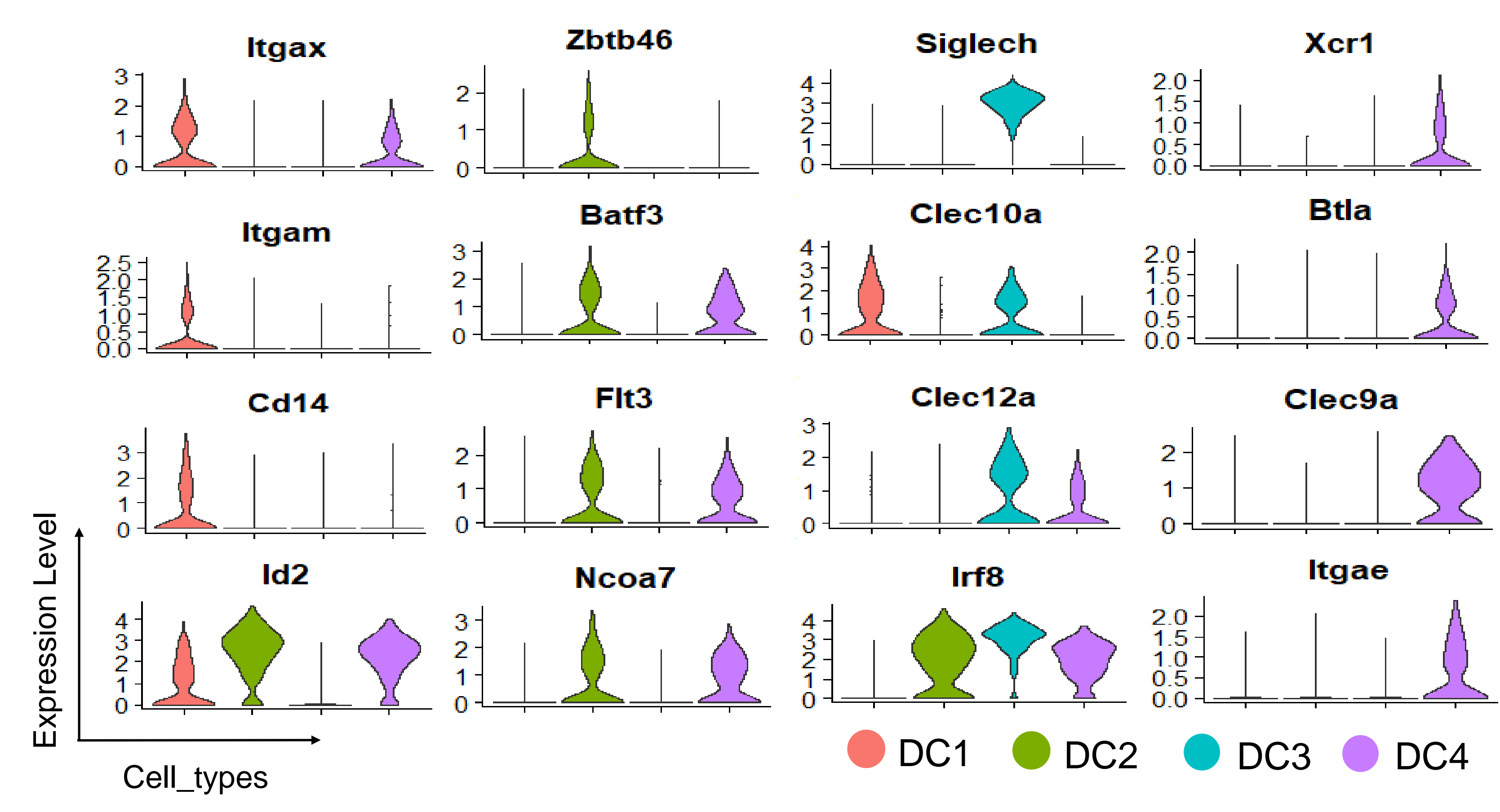

C
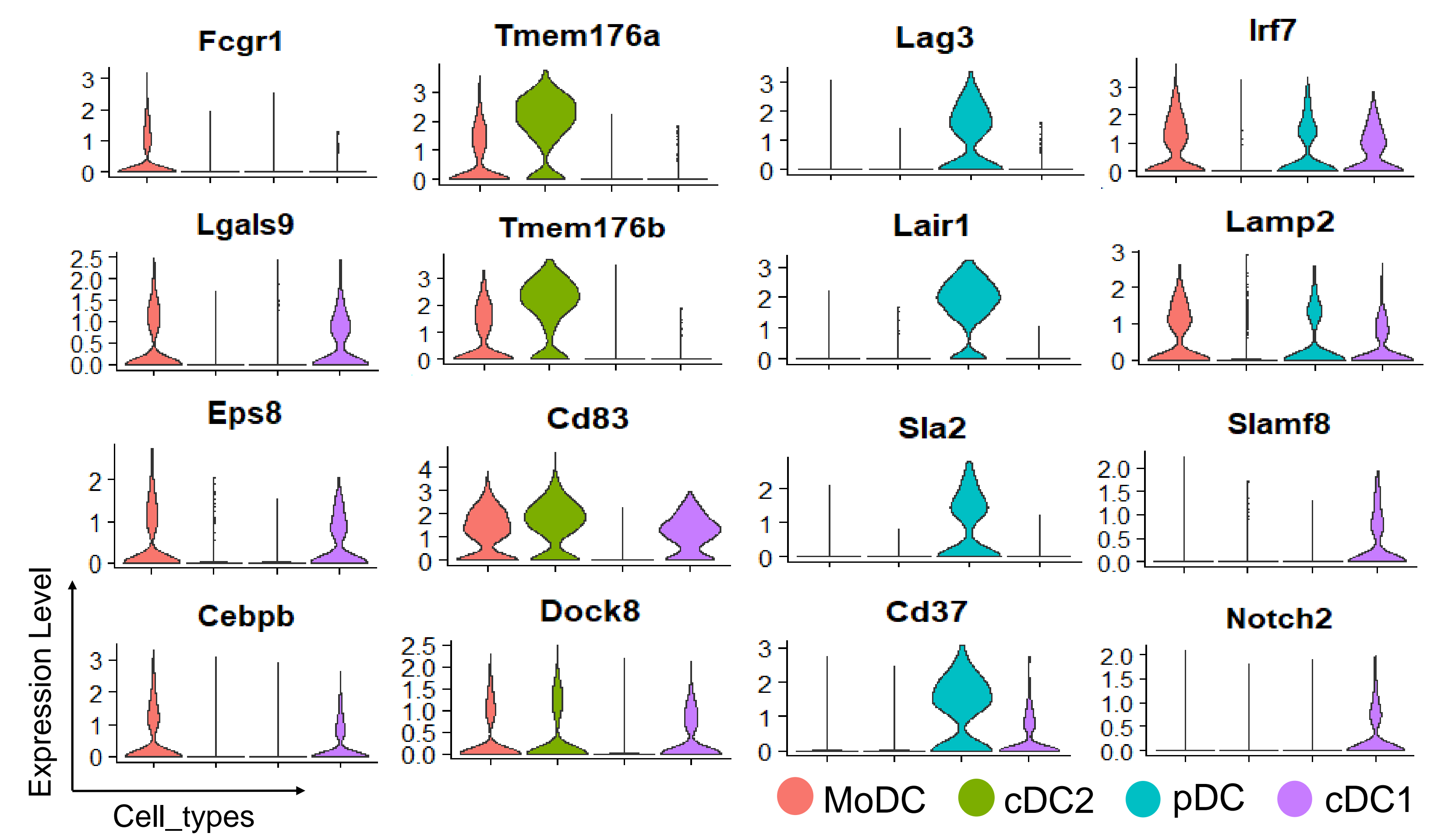

$\mathrm{F}$

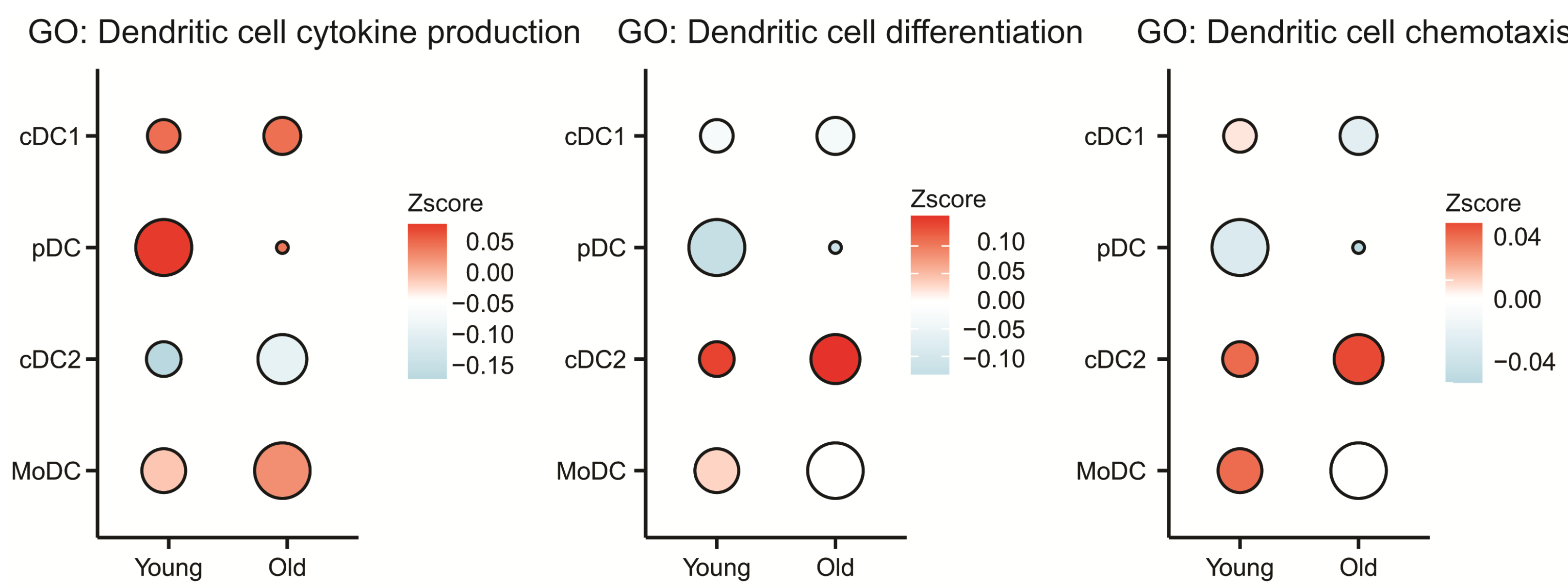

GO: Positive regulation of dendritic cell GO: Negative regulation of dendritic antigen processing and presentation cell apoptotic process

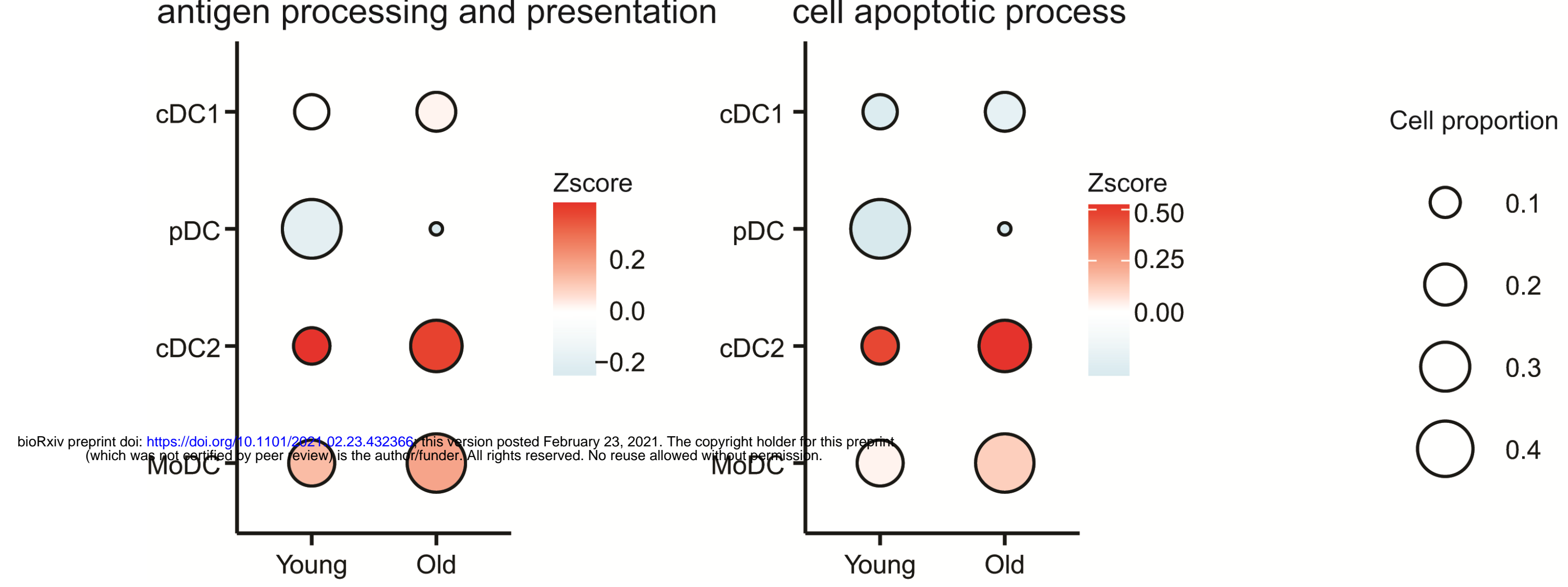

B

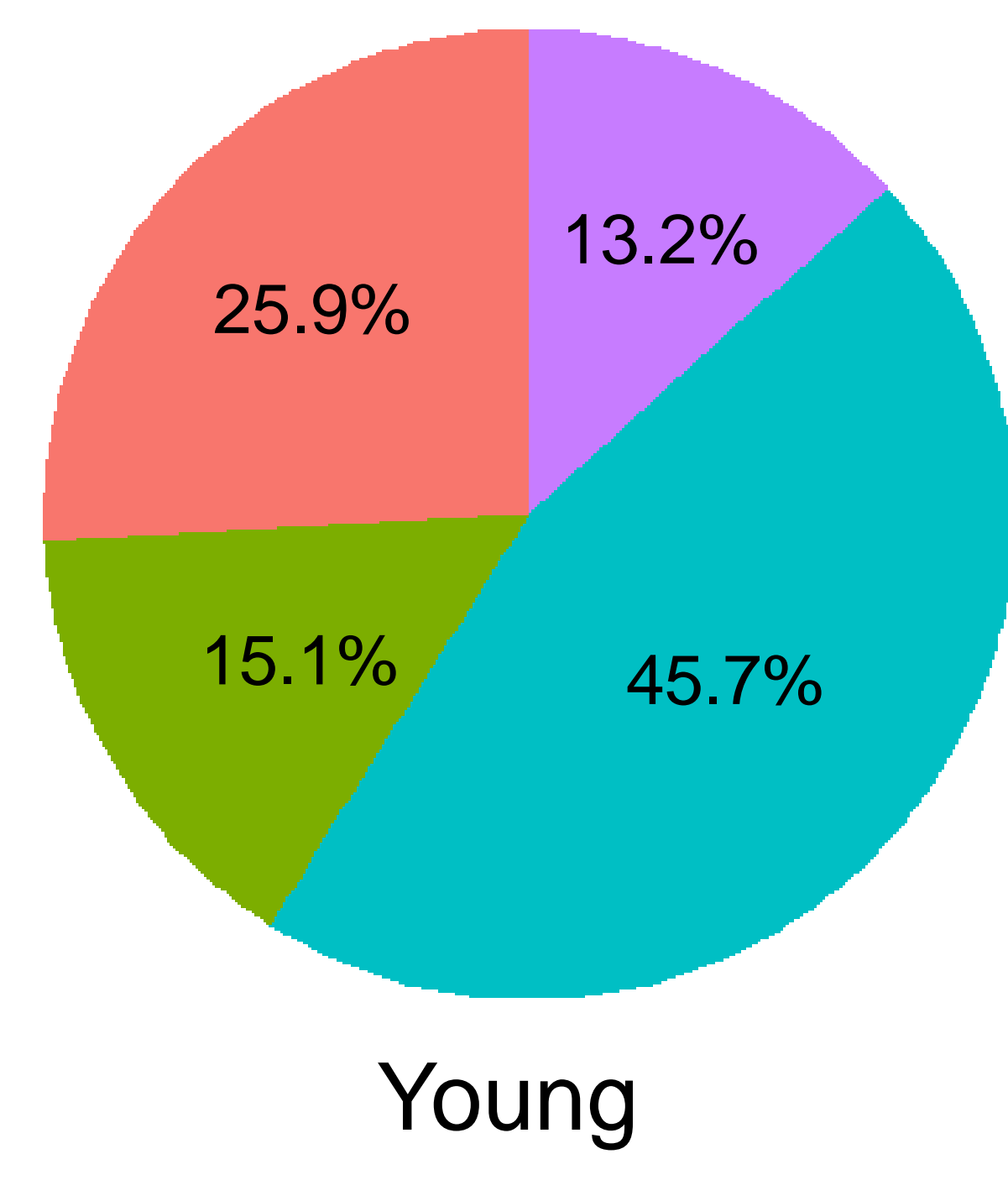

D

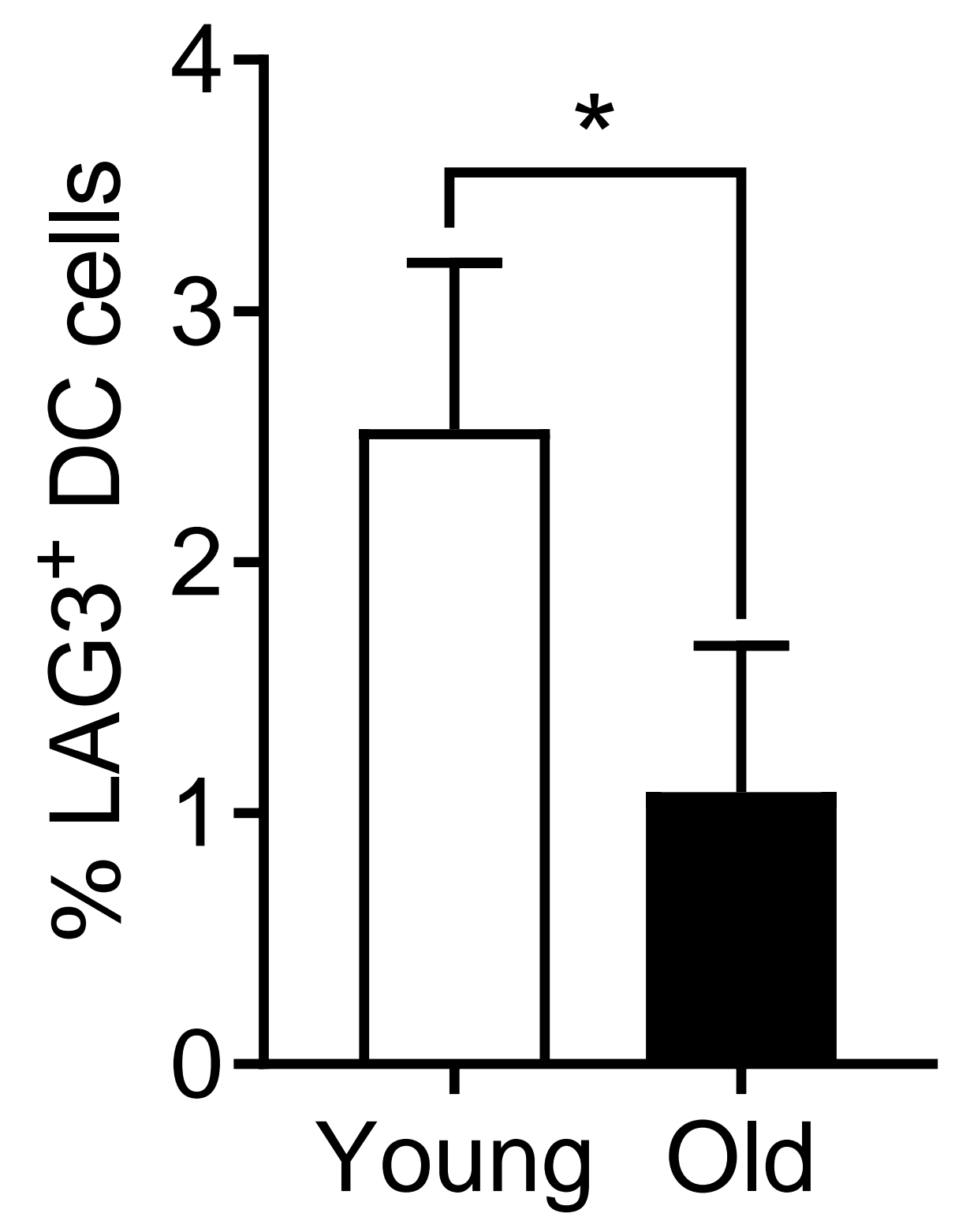

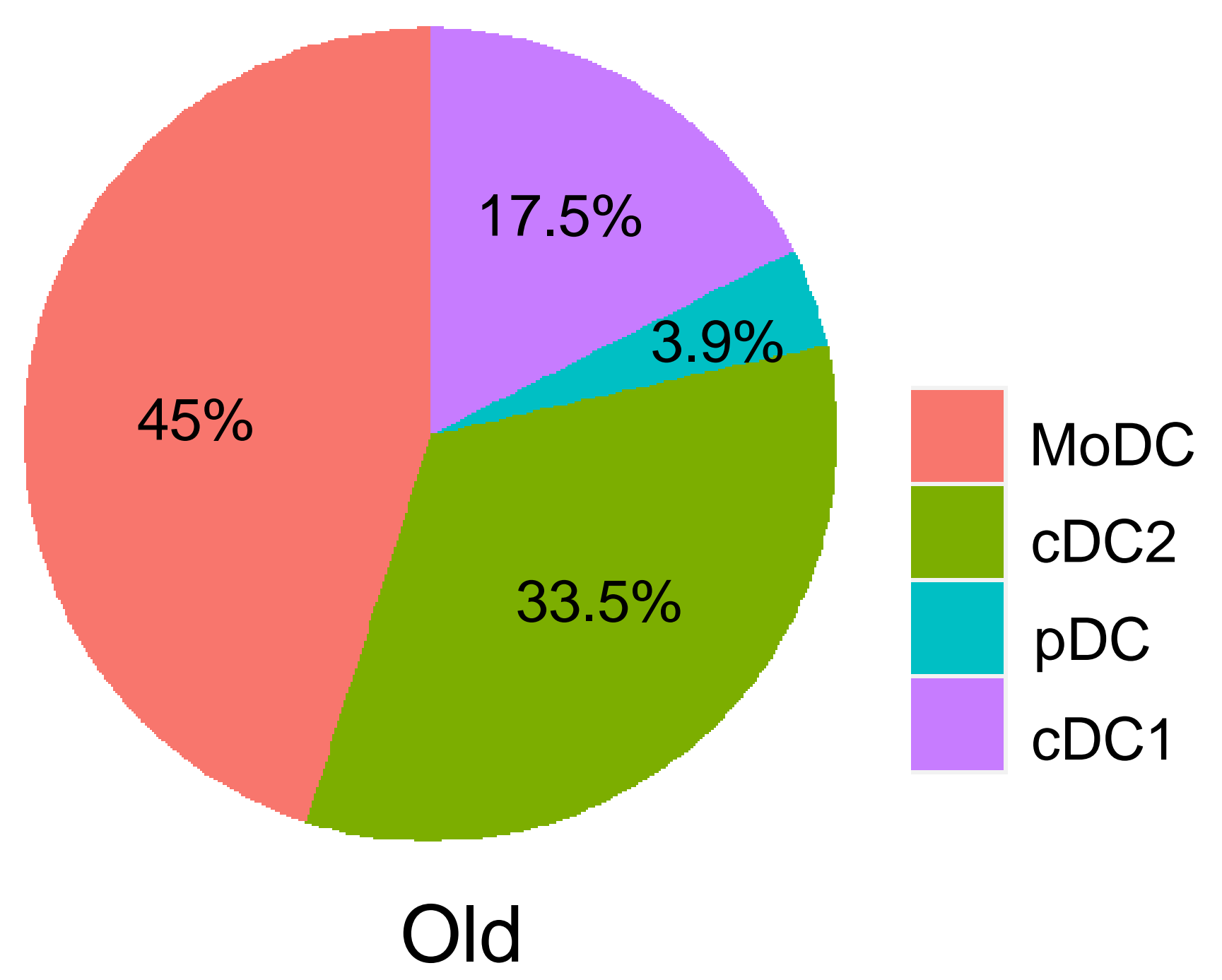

$E$
G
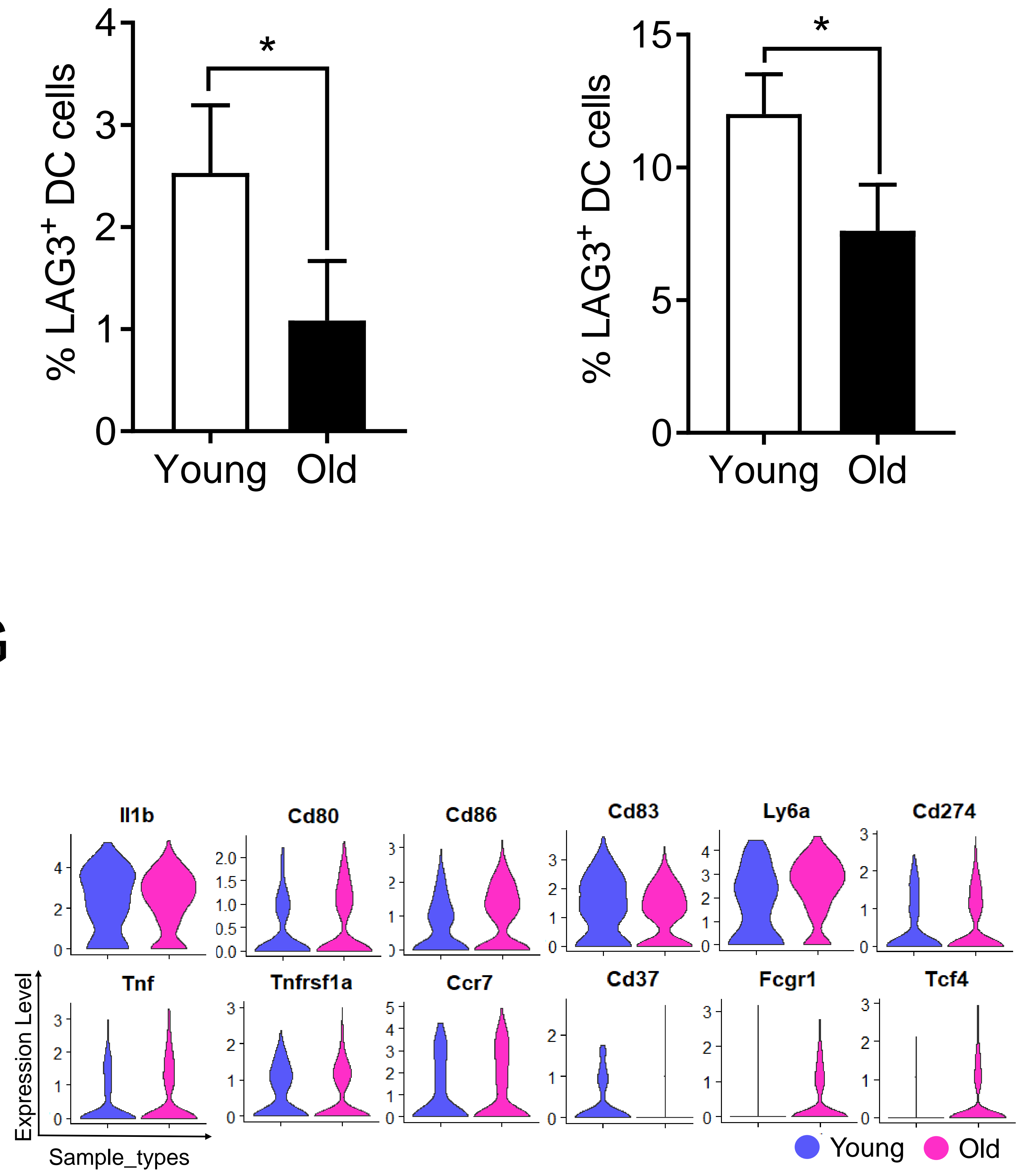
A
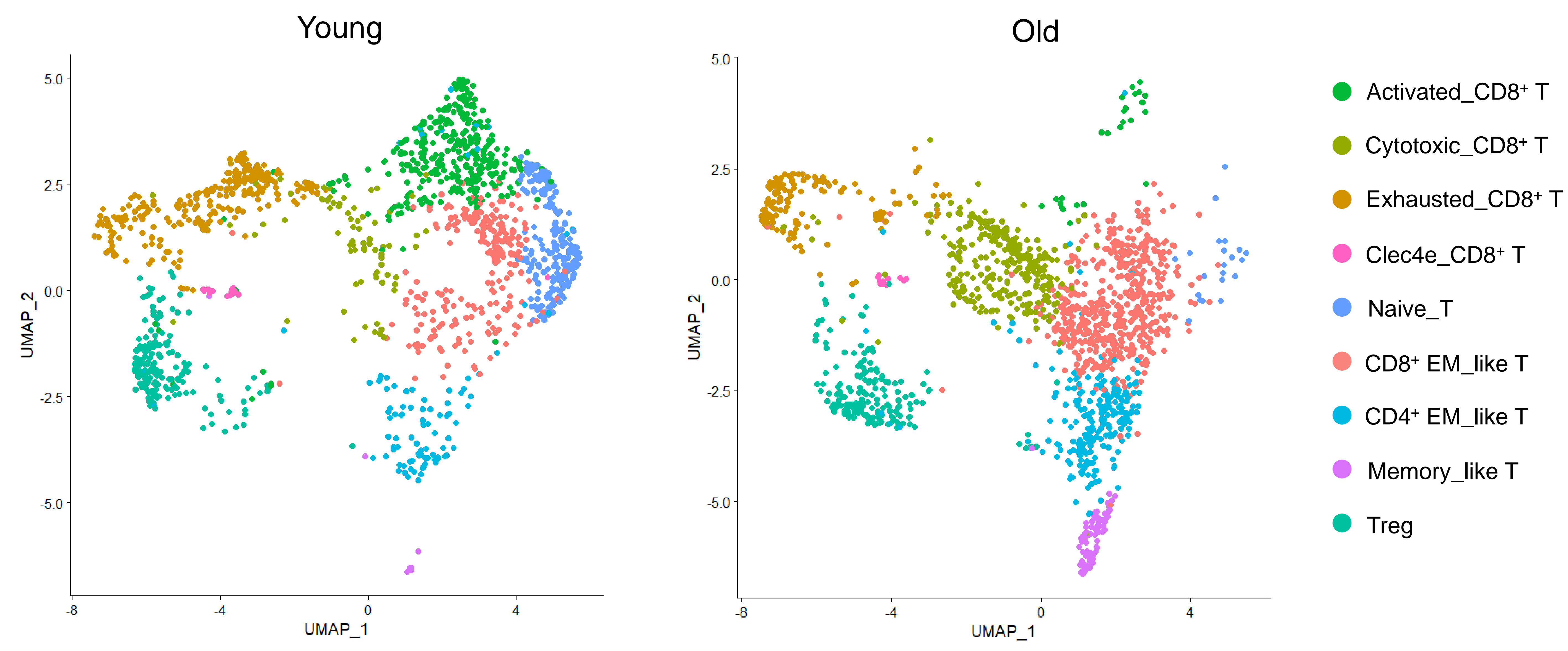

B
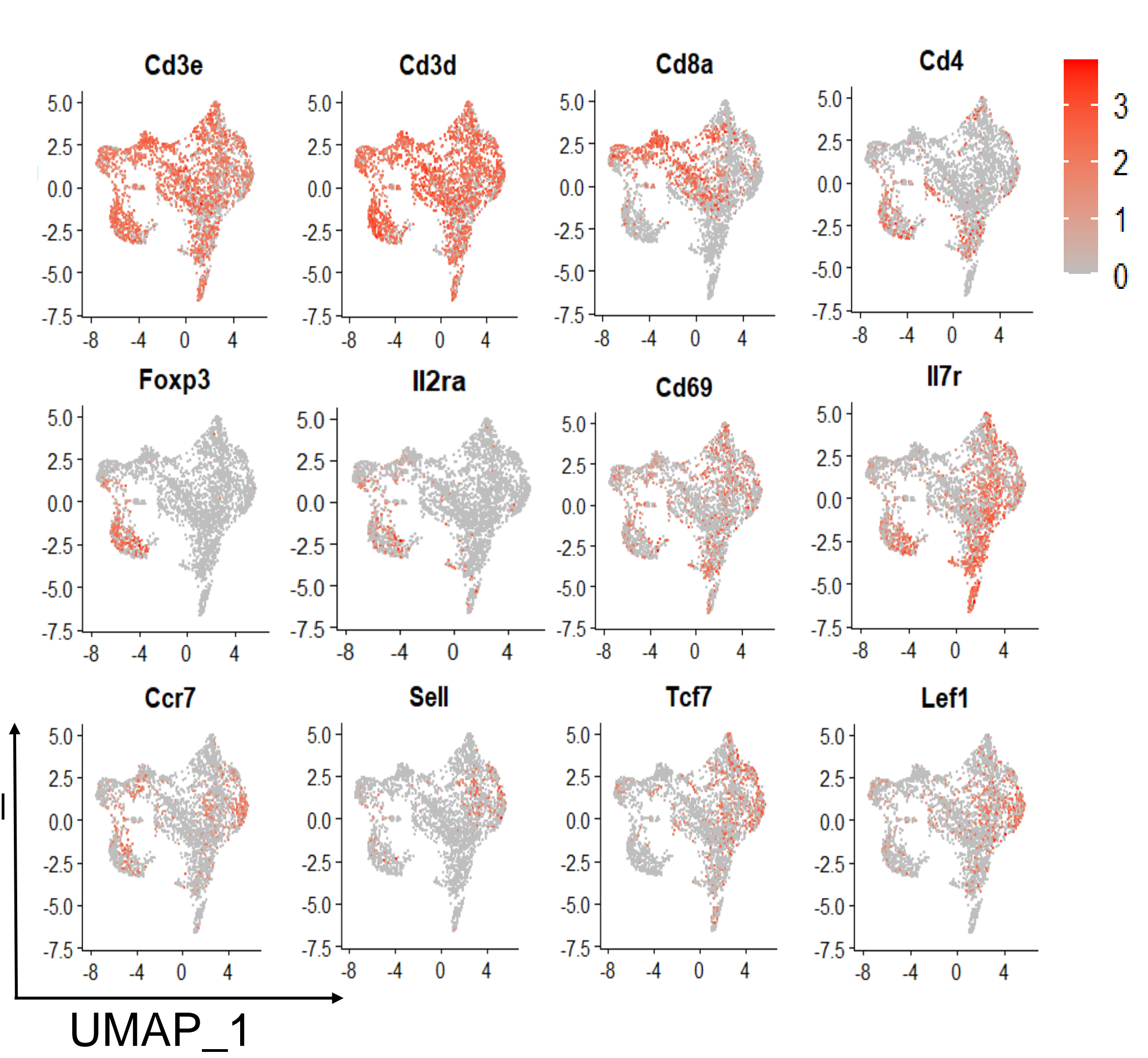

Lef1

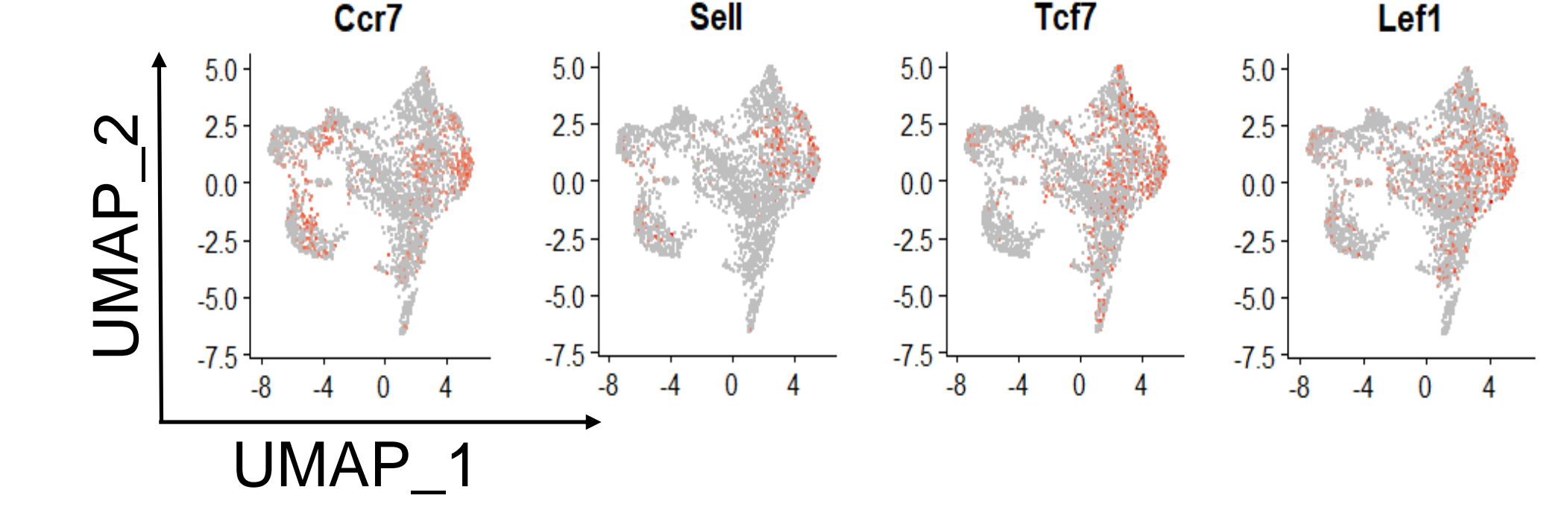

C
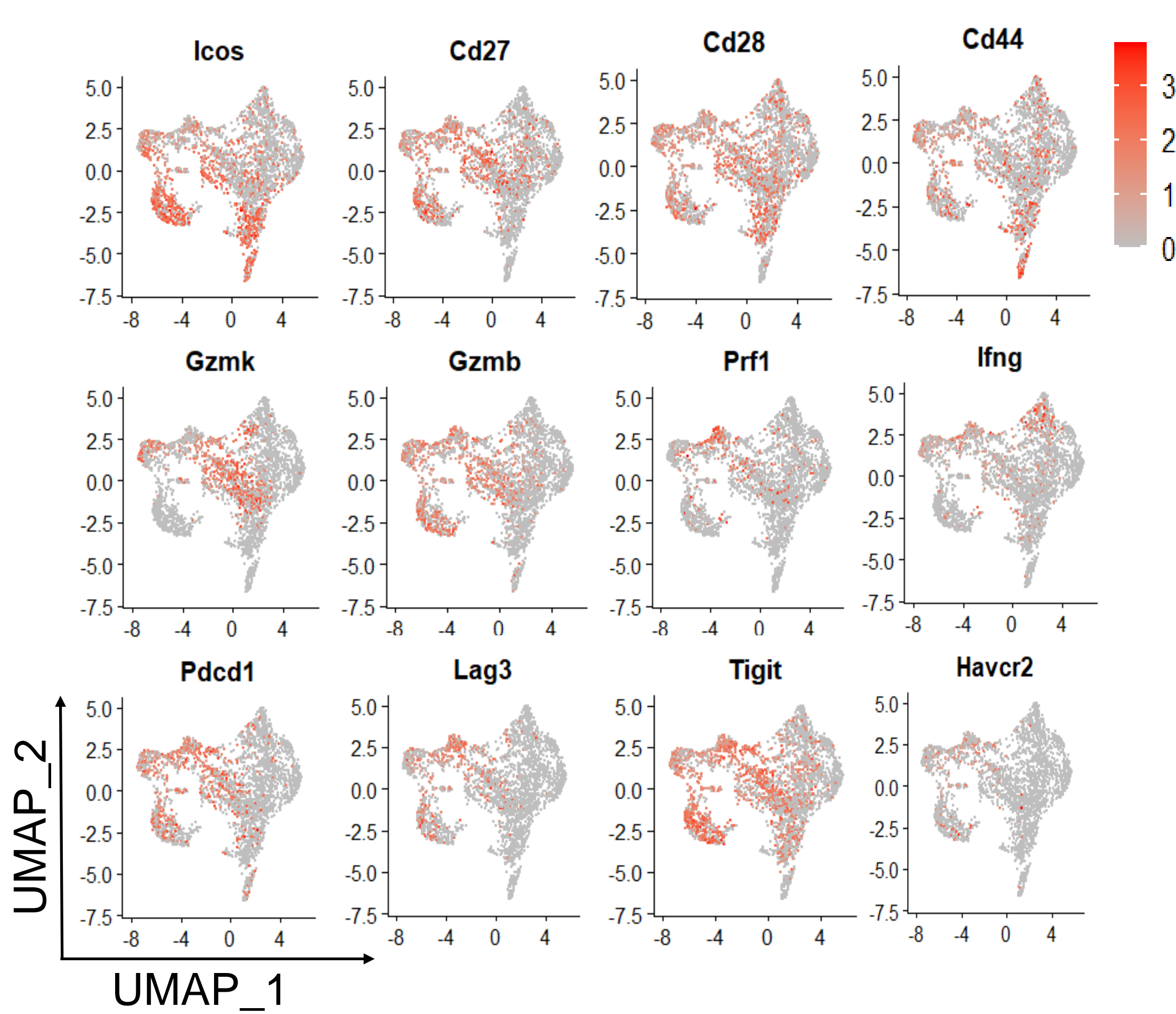

D

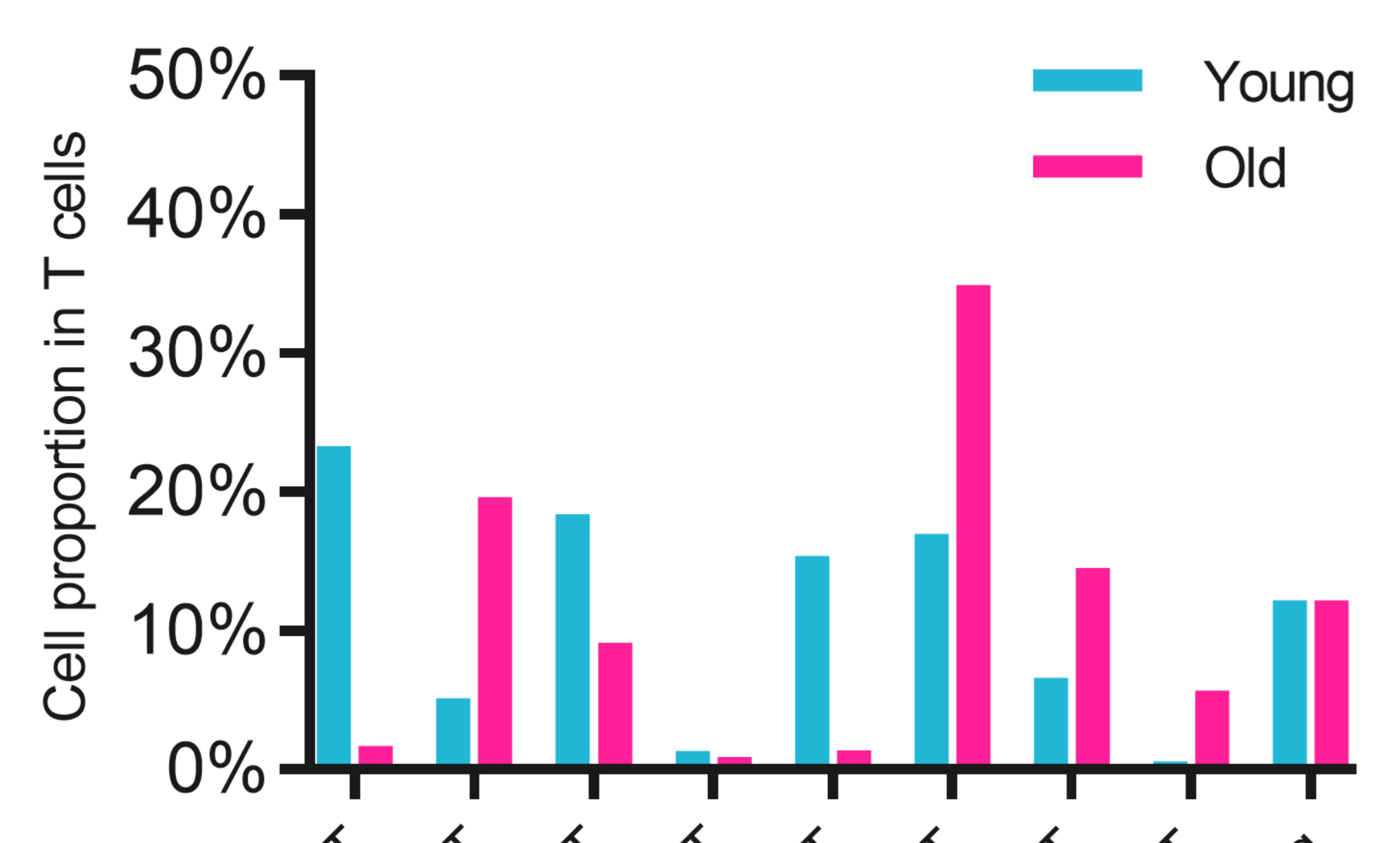

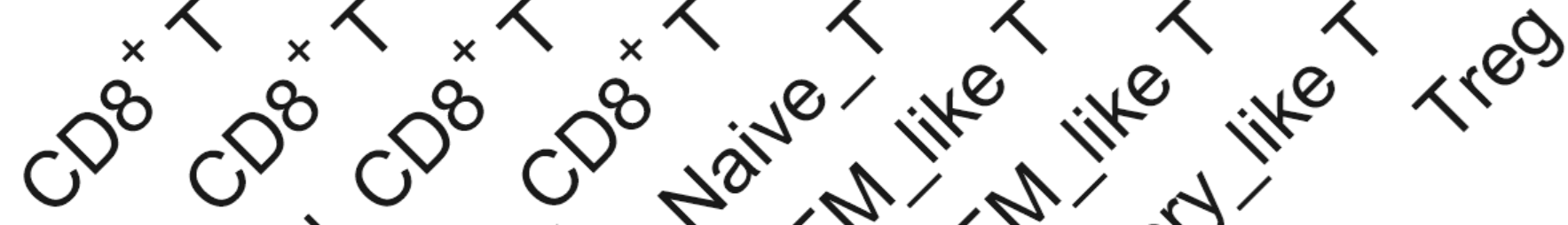

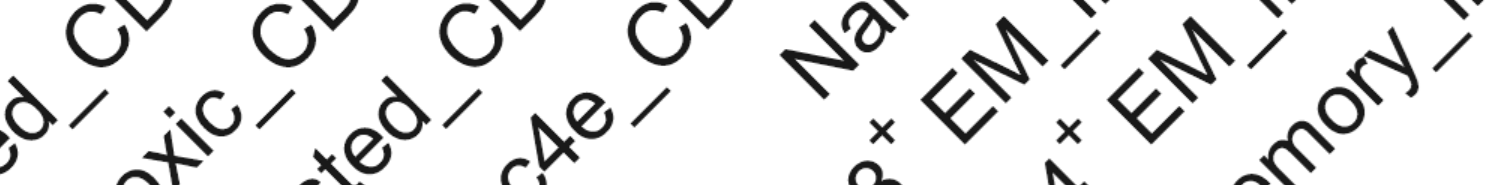

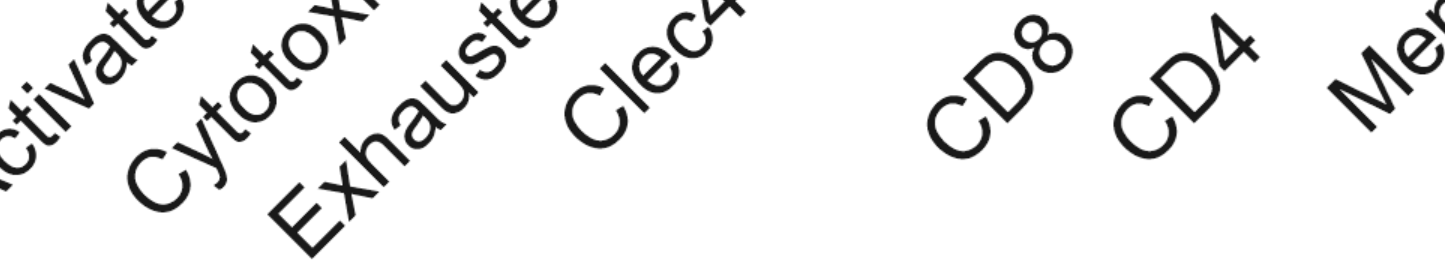


Fig. 6

A

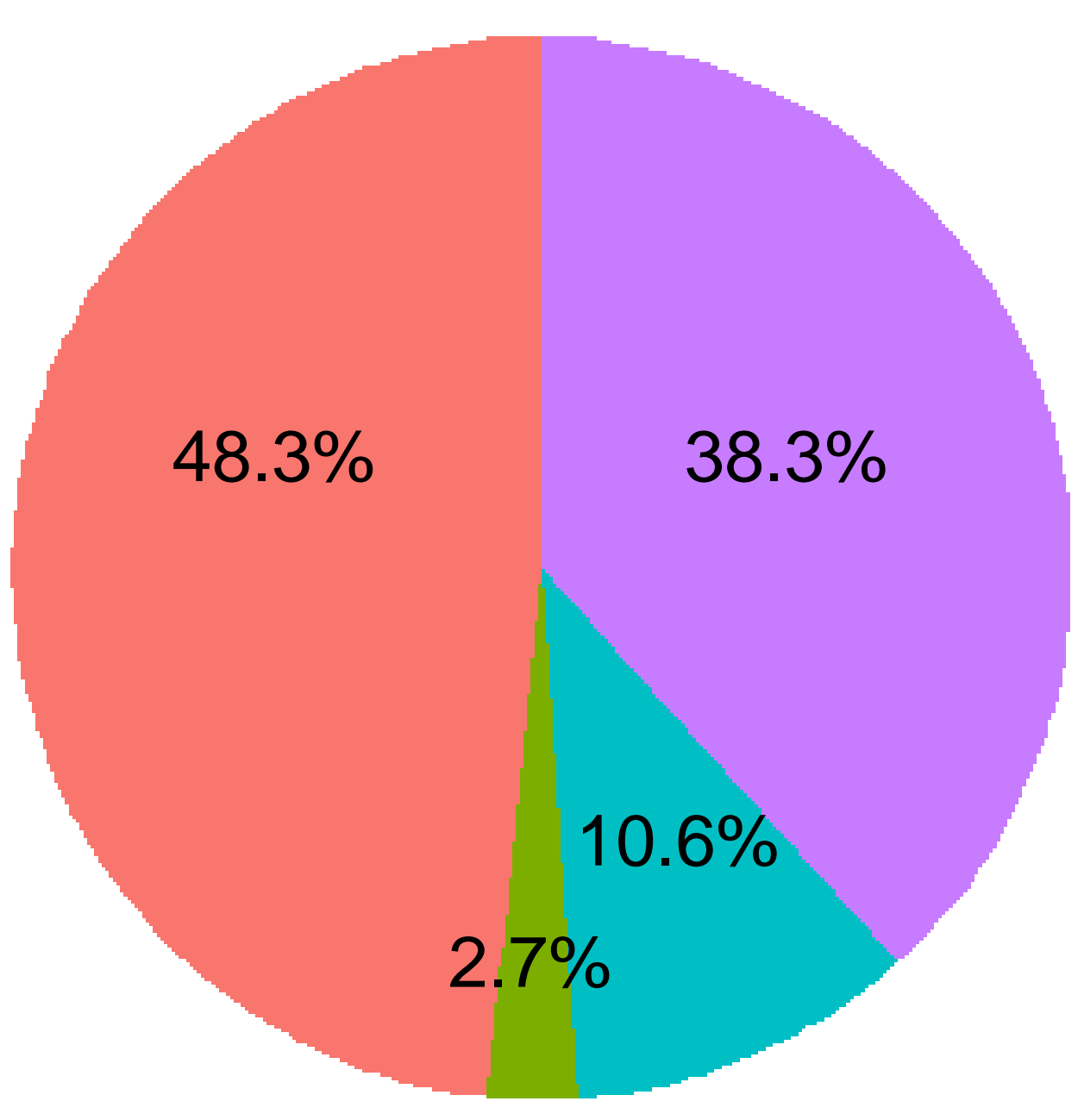

Young

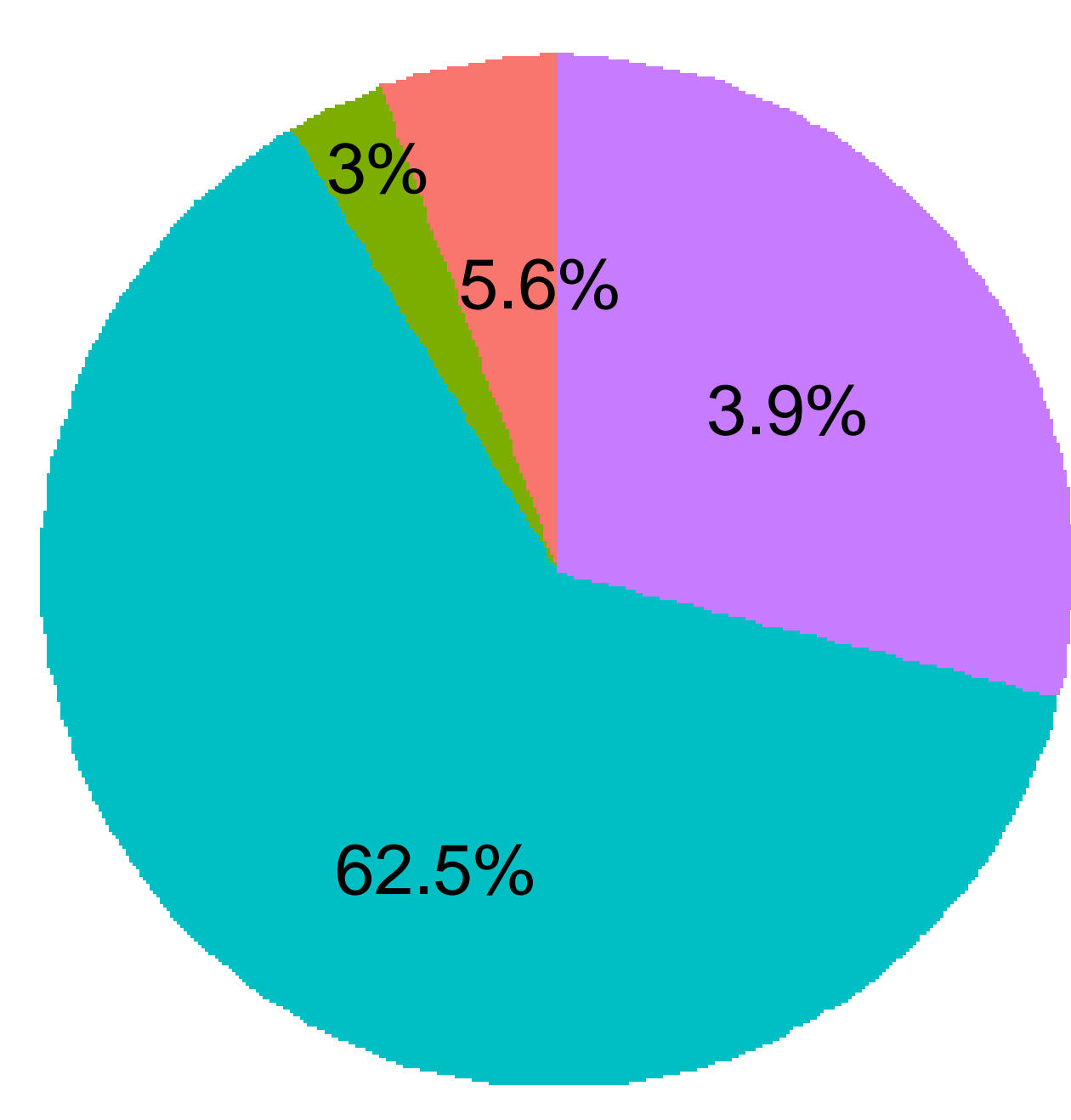

Old
B

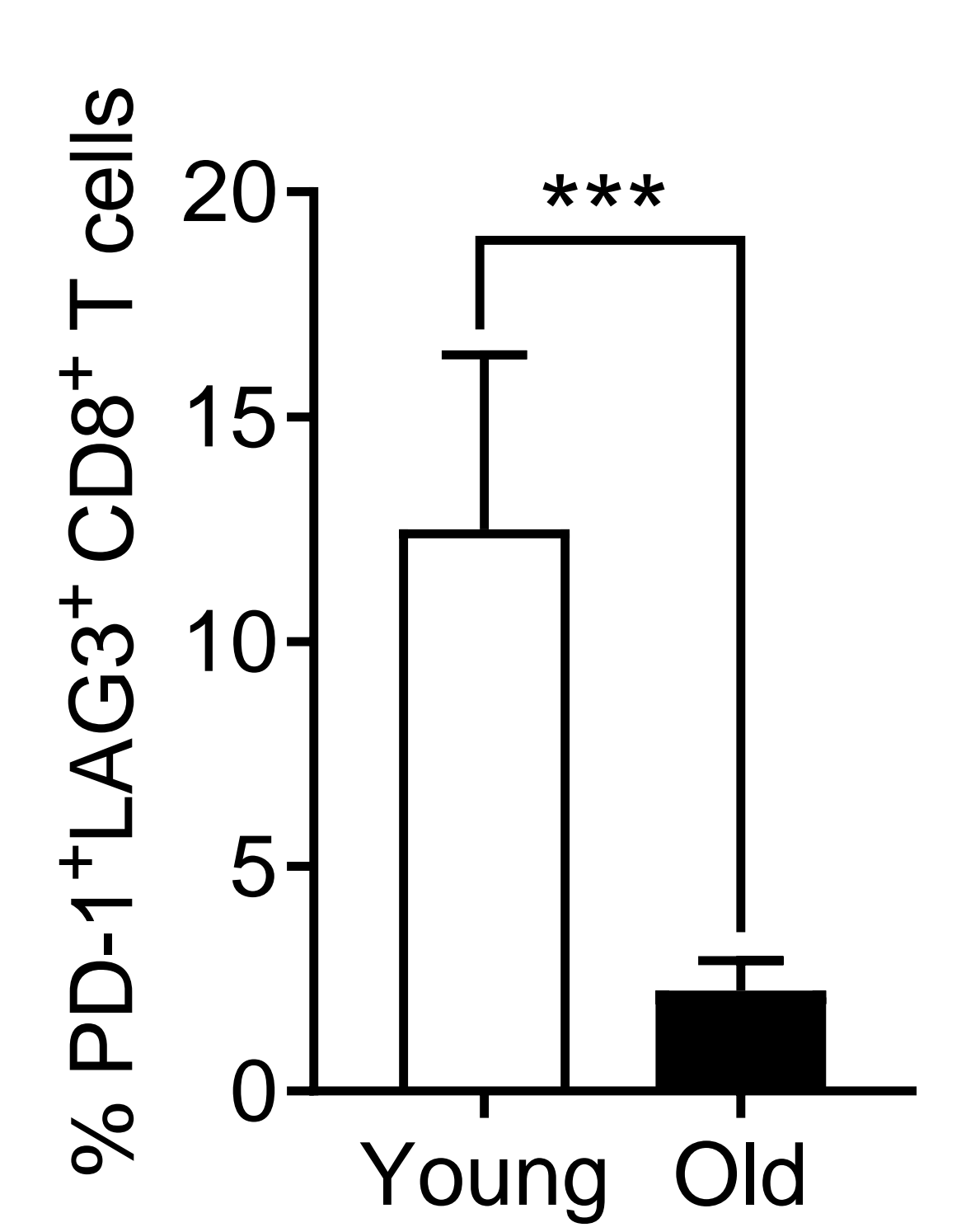

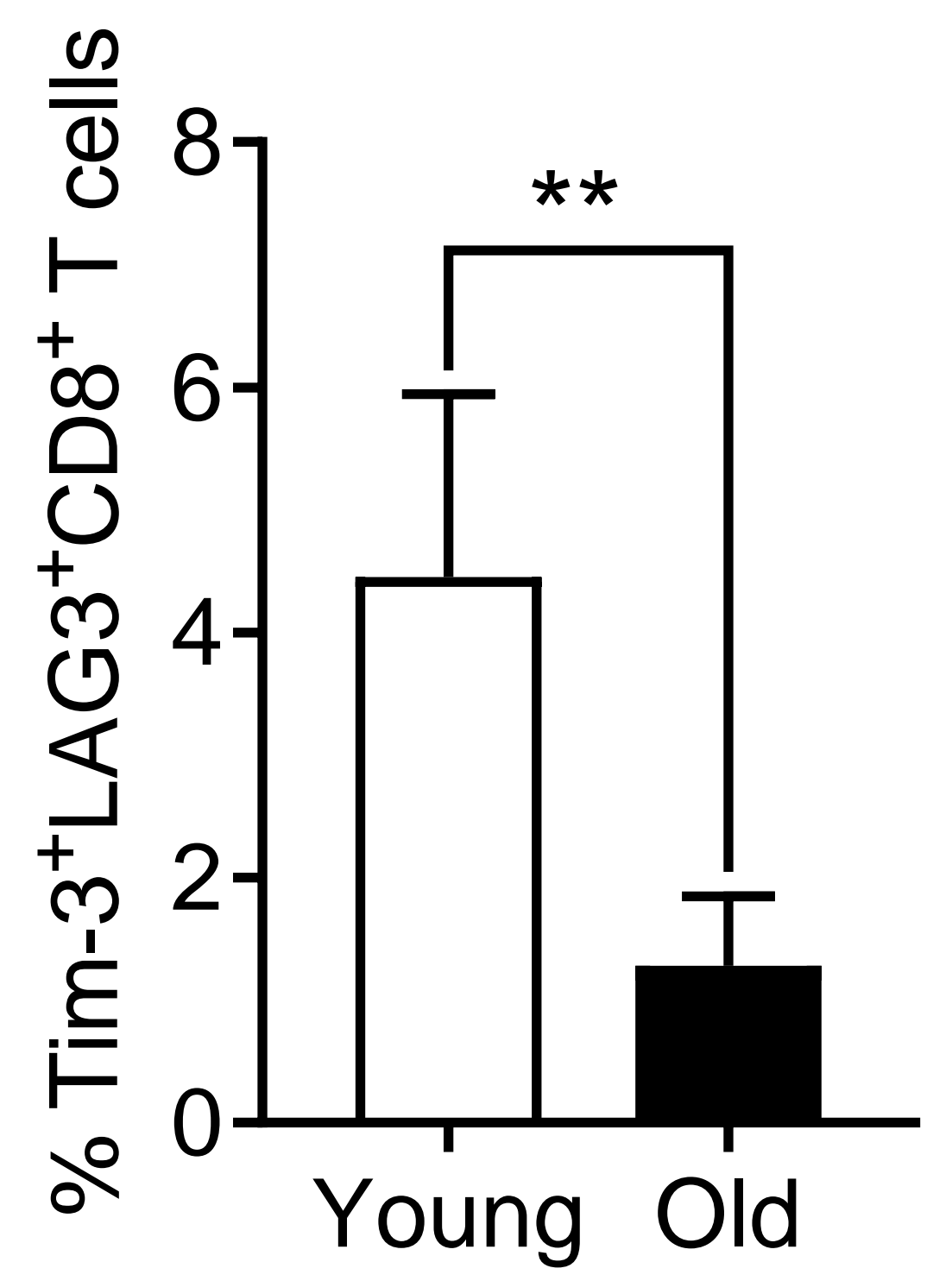

C

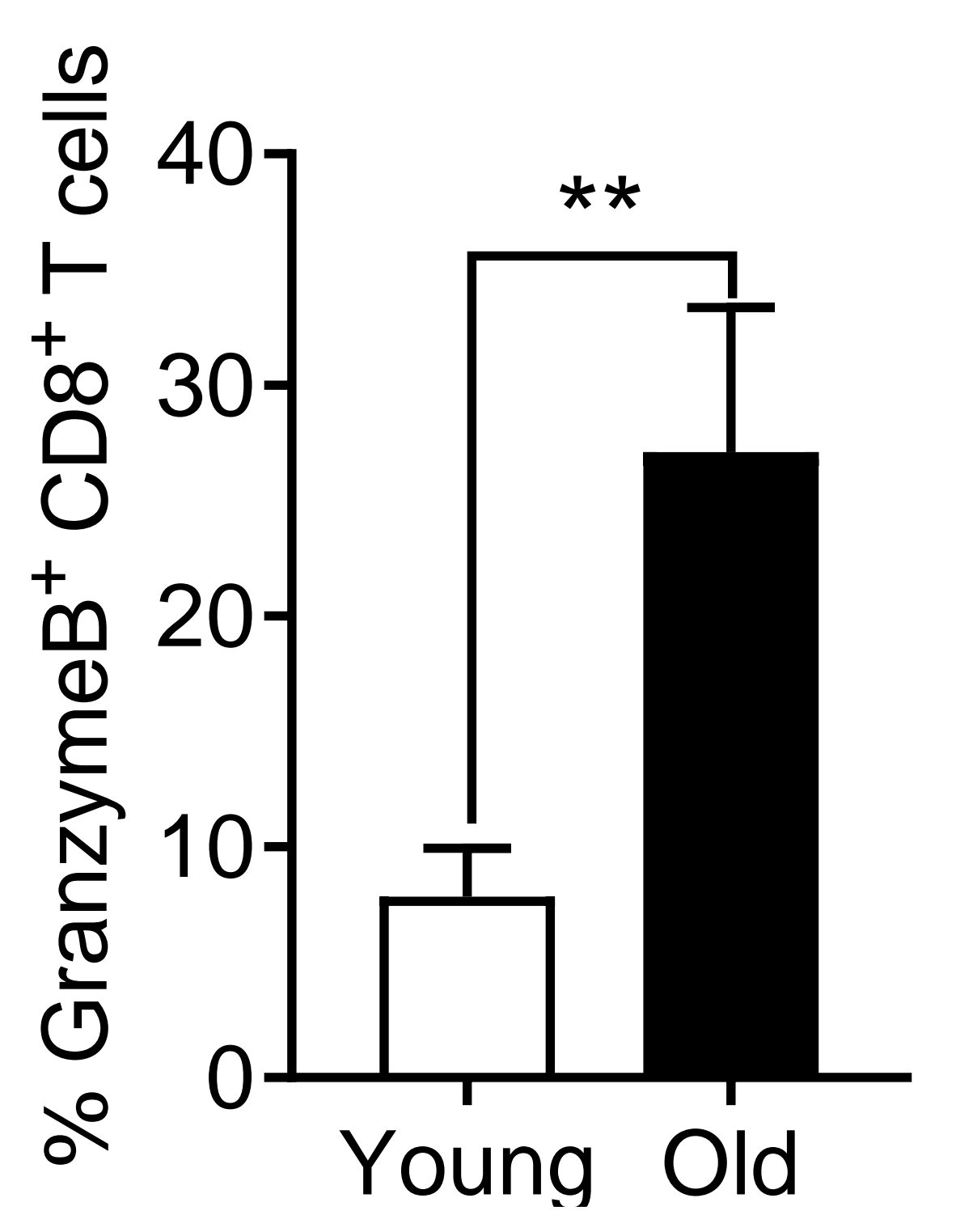

$E$

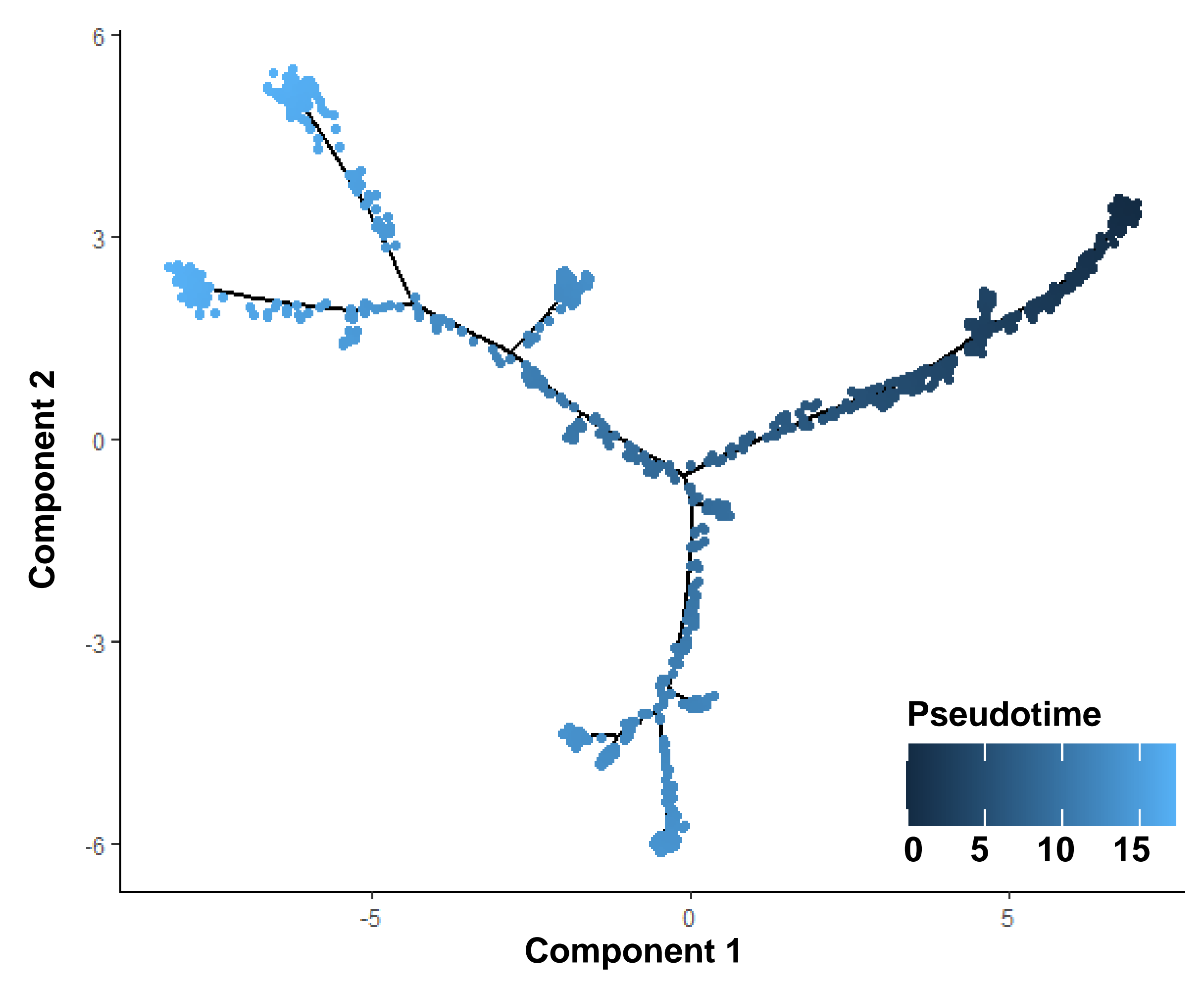

G

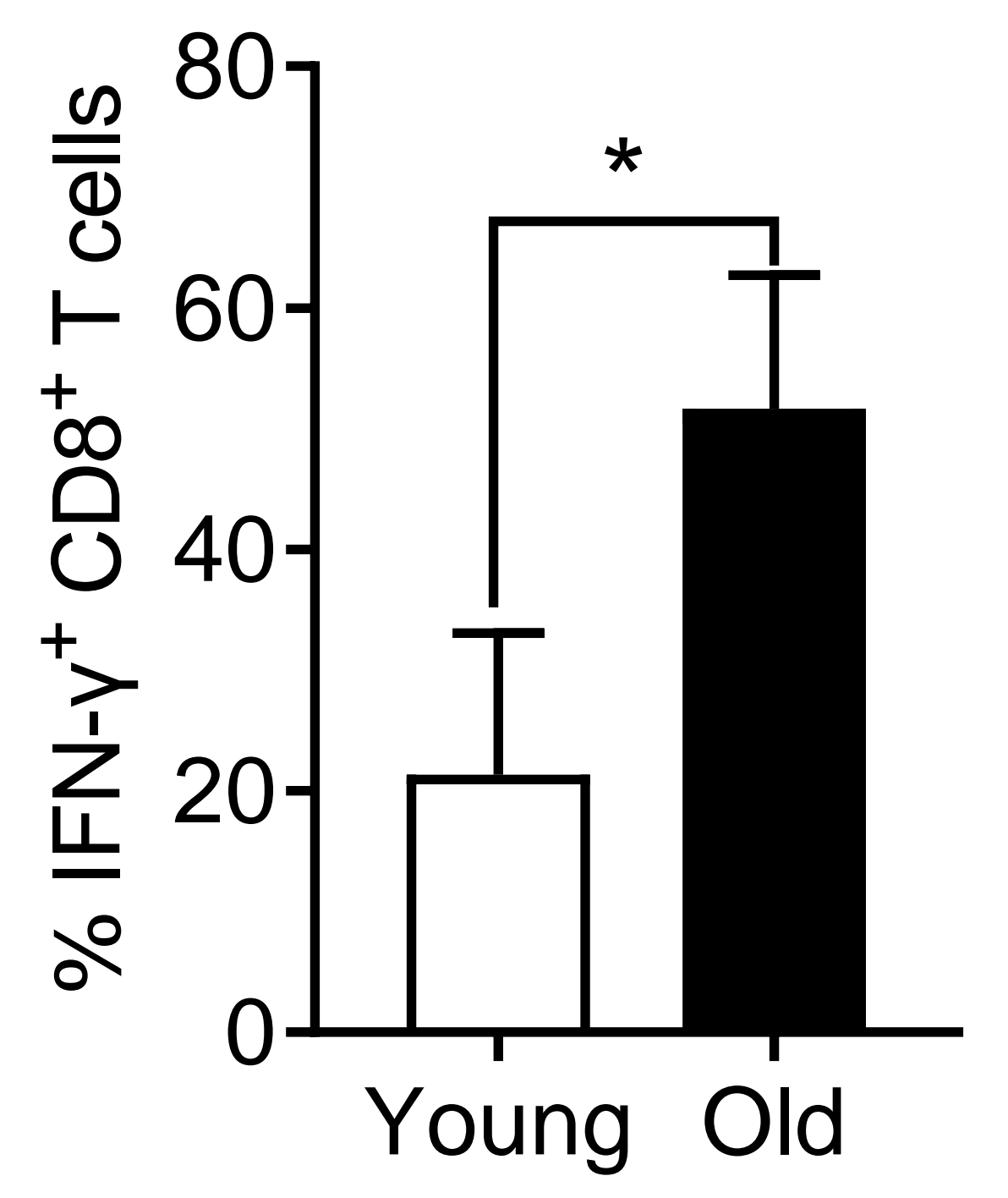

Clec4e CD8 ${ }^{+} T$

Cytotoxic_CD8 ${ }^{+} \mathrm{T}$

Exhausted_CD8 ${ }^{+} T$

D
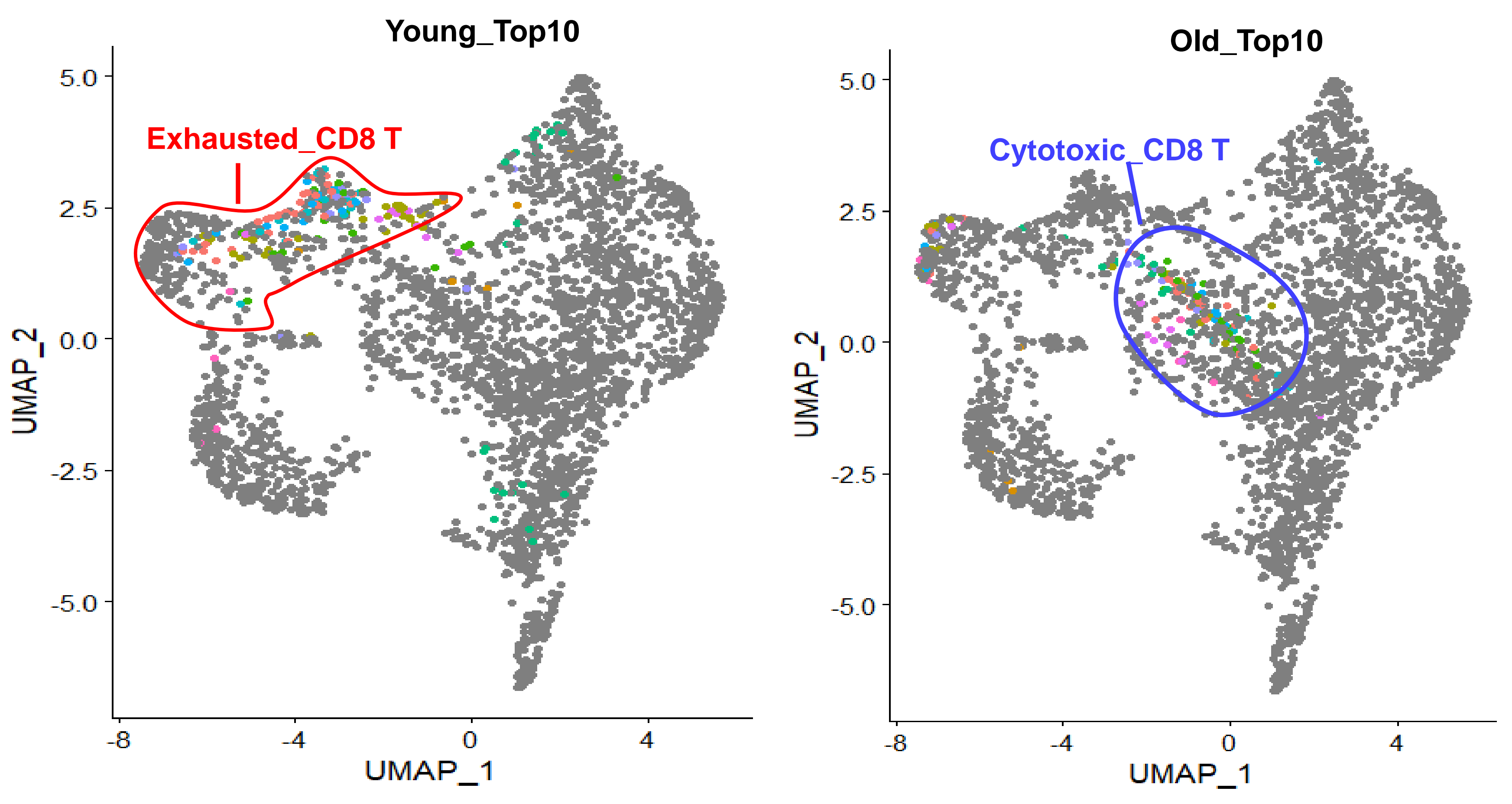

F

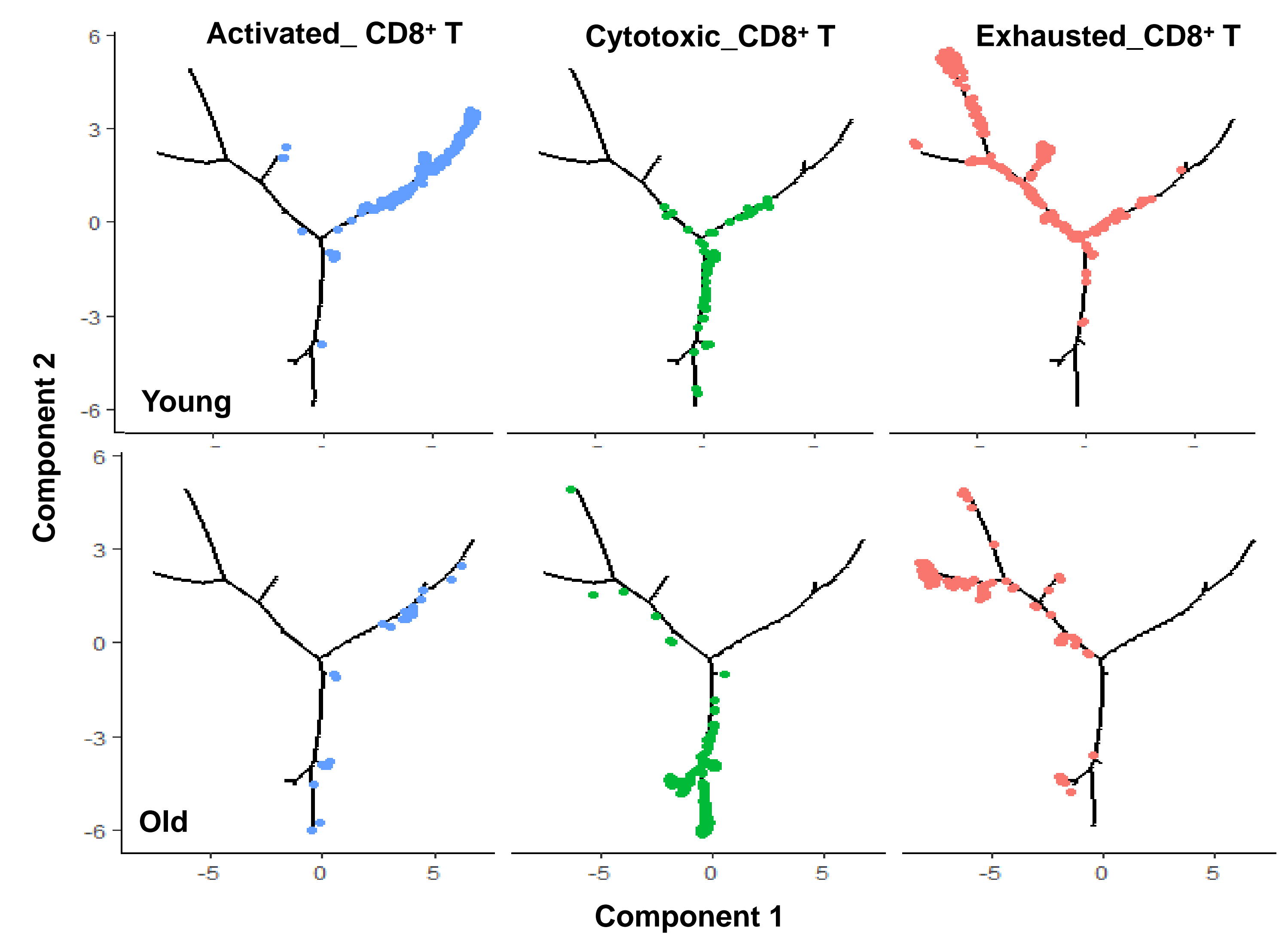

$\mathrm{H}$

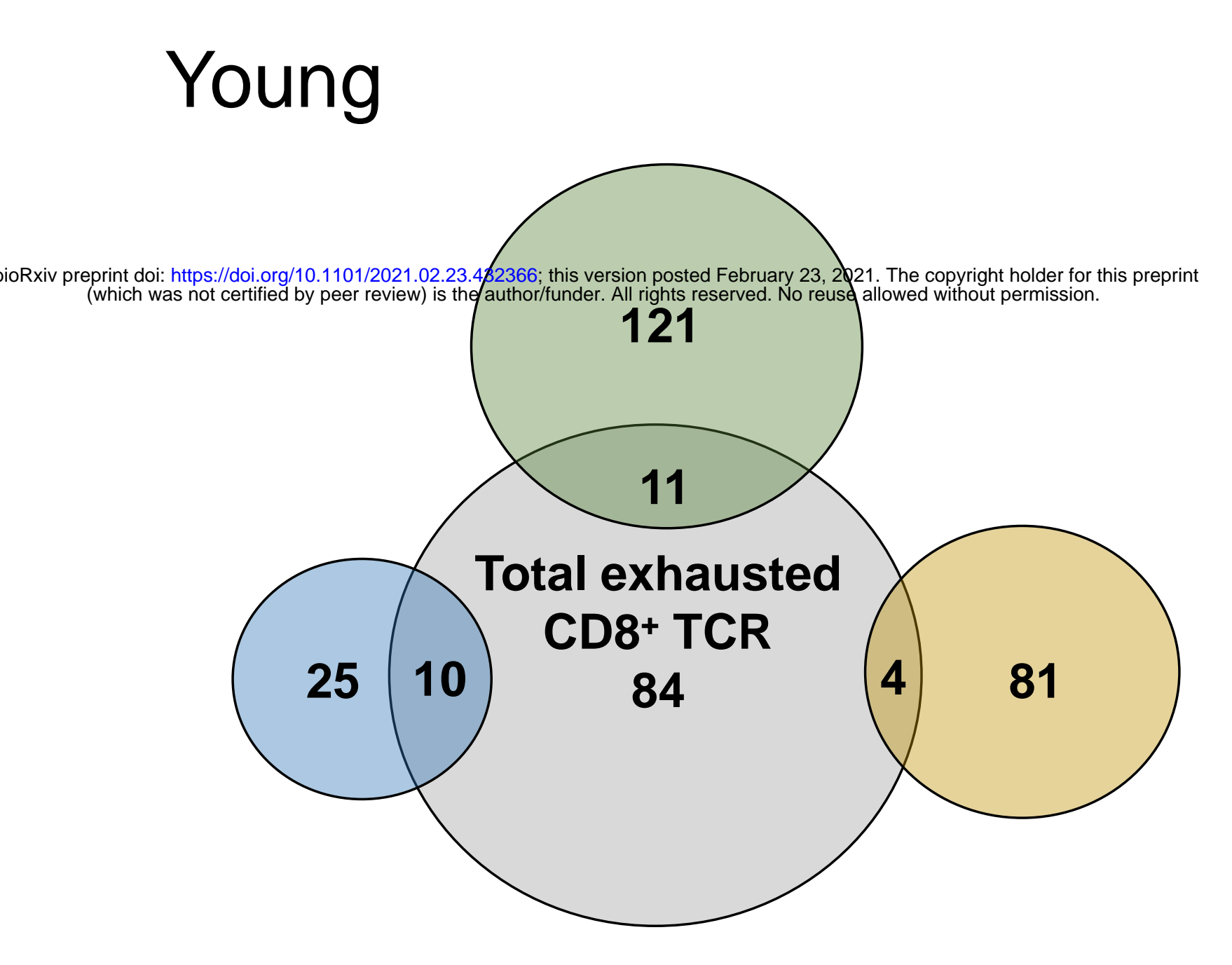

Old

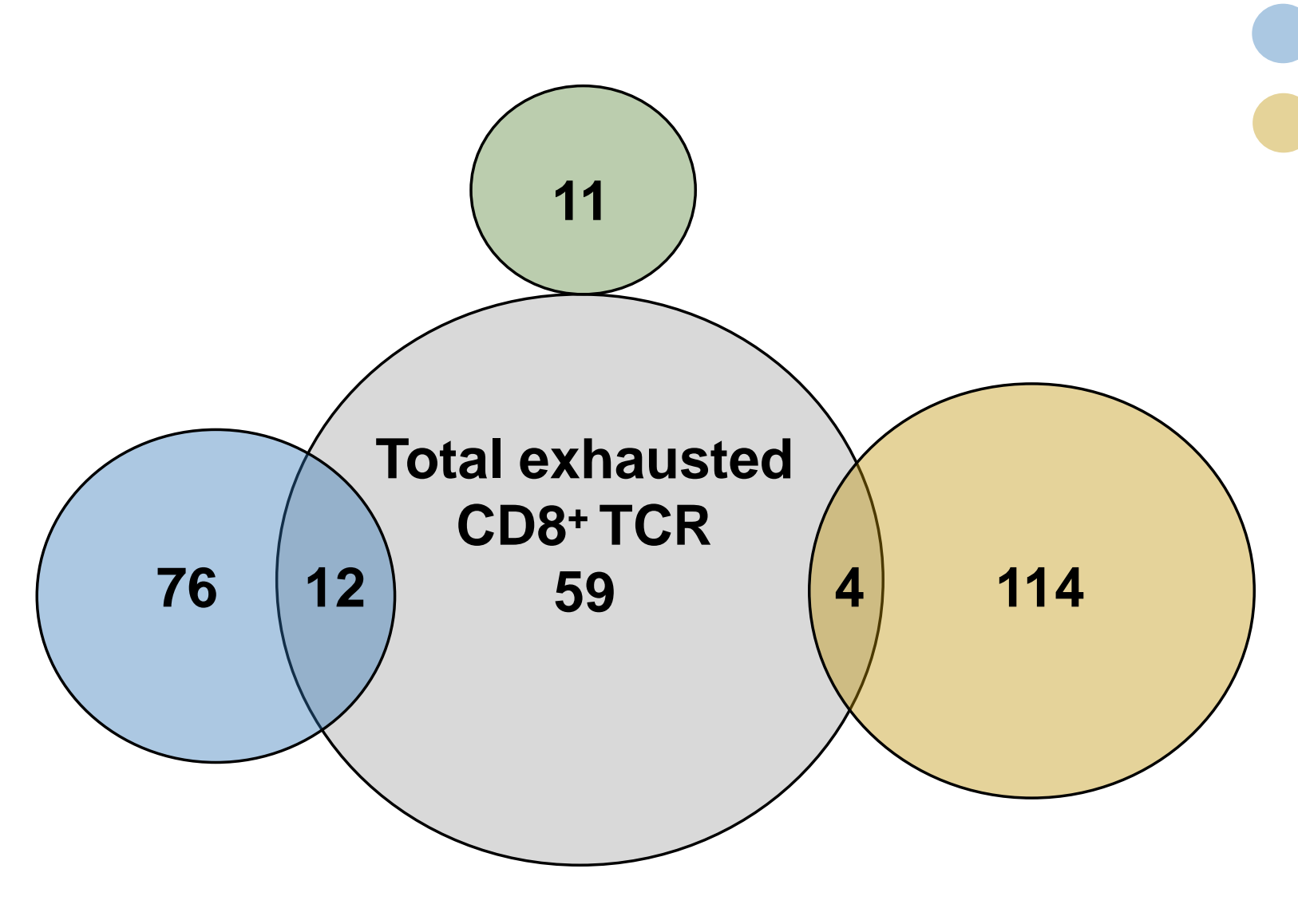

Activated_CD8 ${ }^{+} \mathrm{T}$

Cytotoxic_CD8 ${ }^{+} \mathbf{T}$

CD8+ EM_like T
Exhausted_CD8+ T

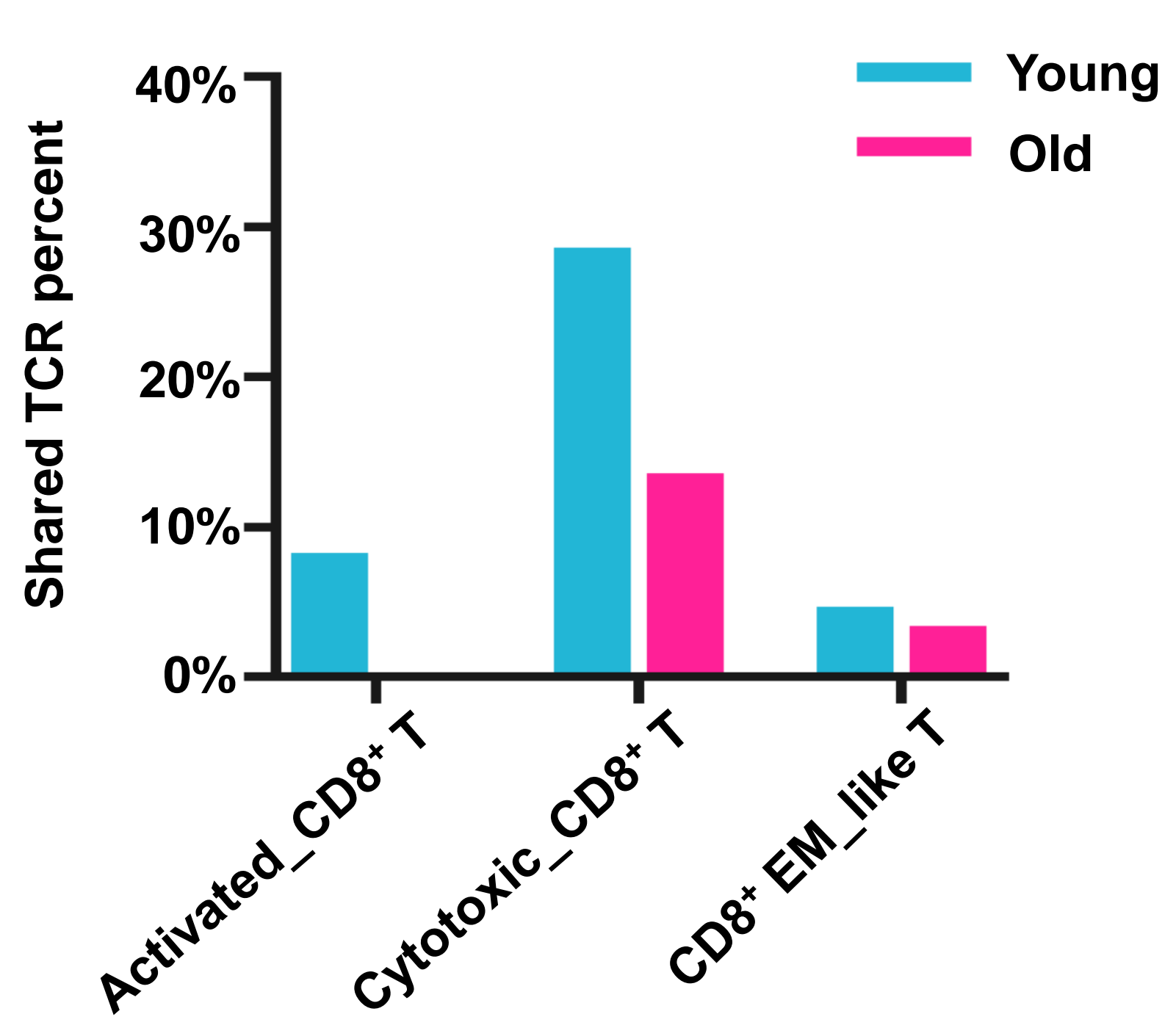


Fig. 7

A

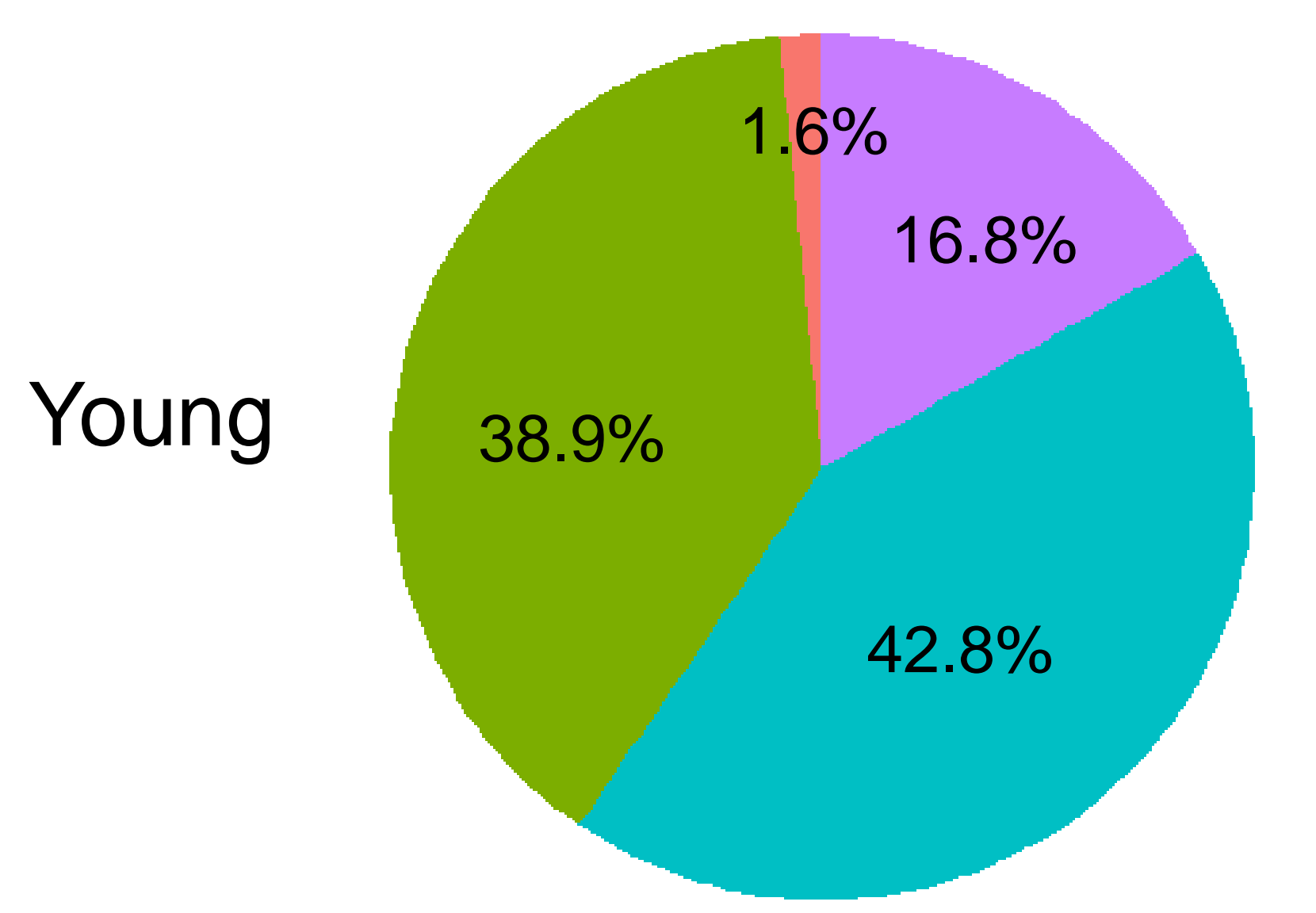

Old

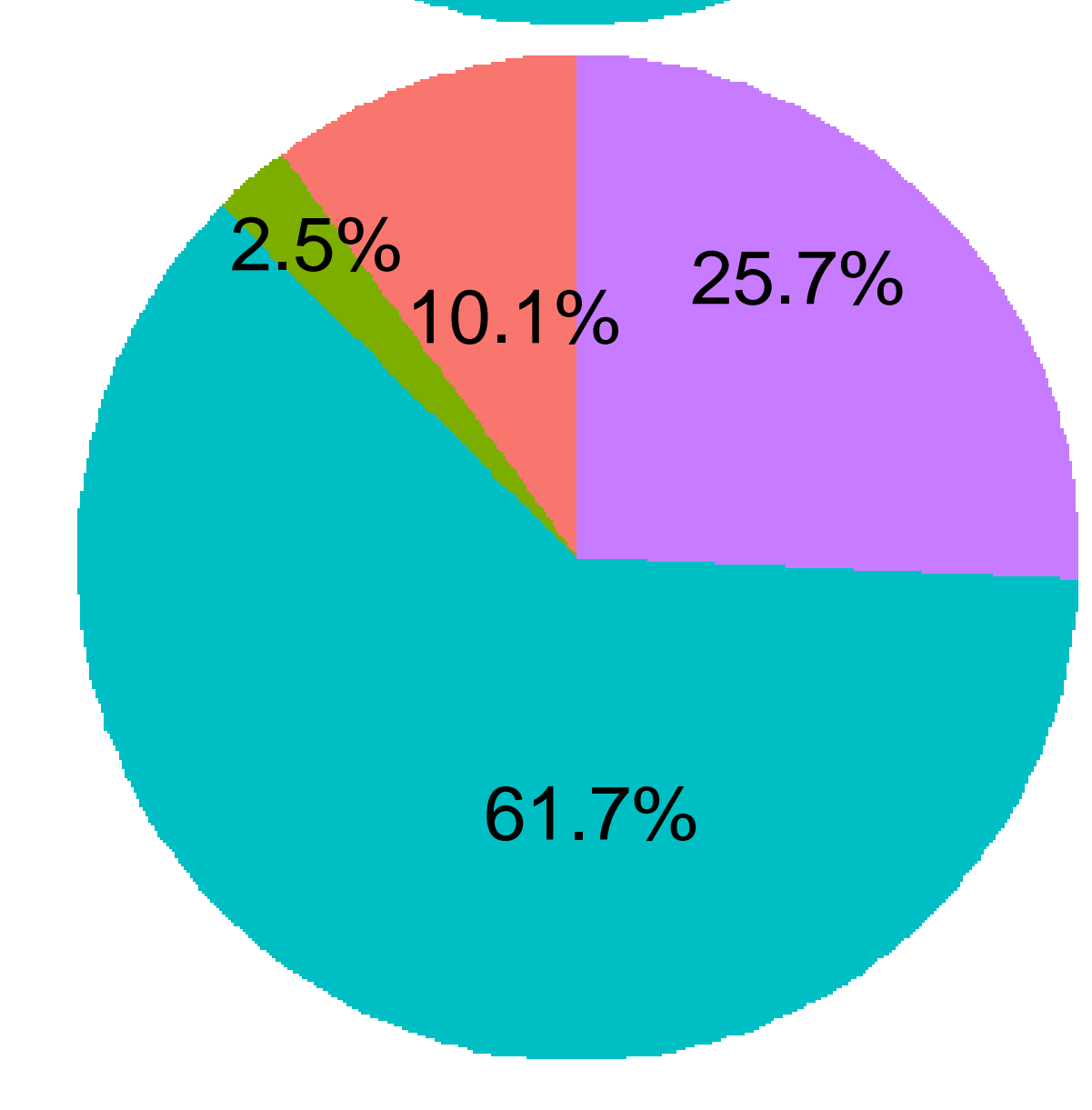

D

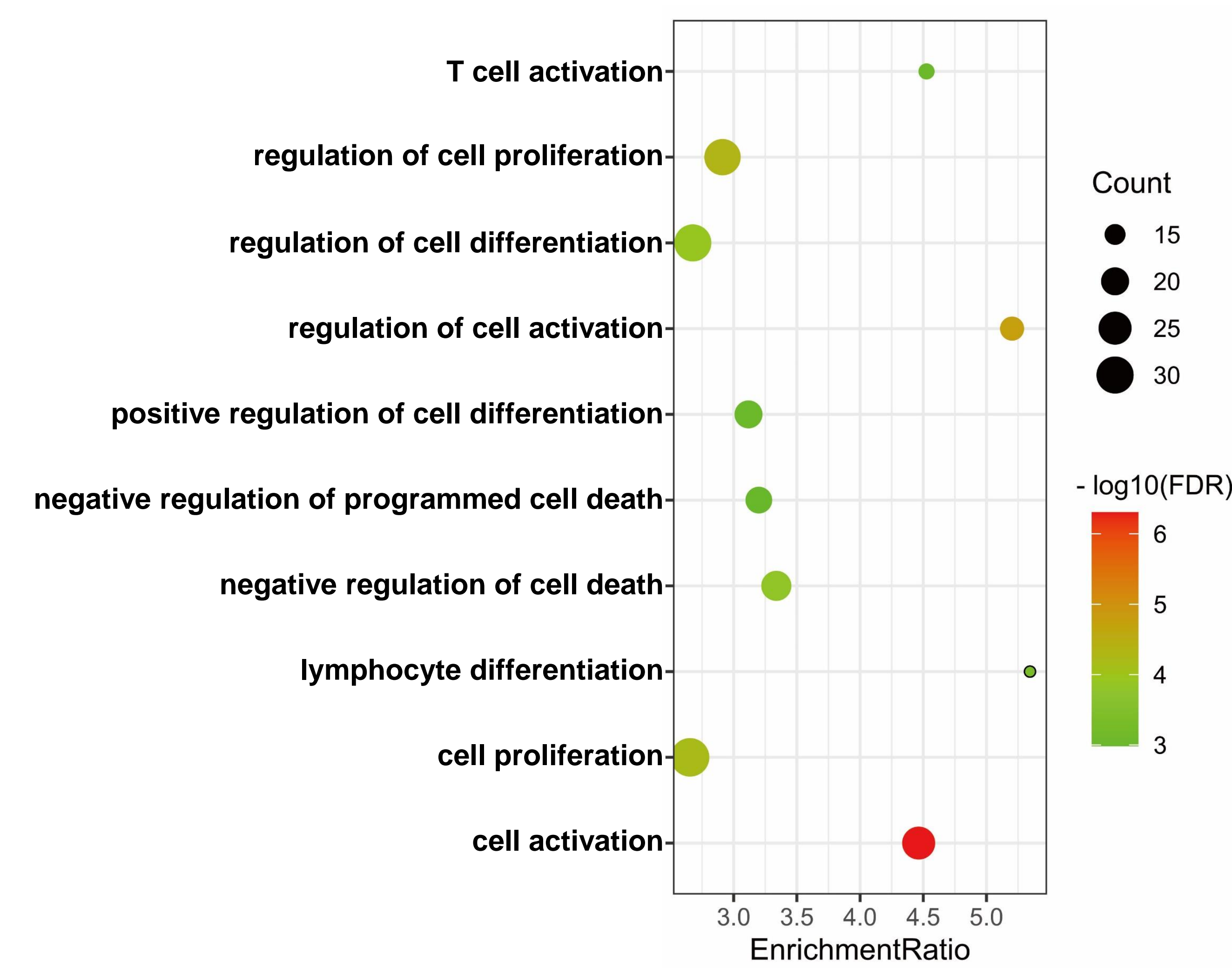

B

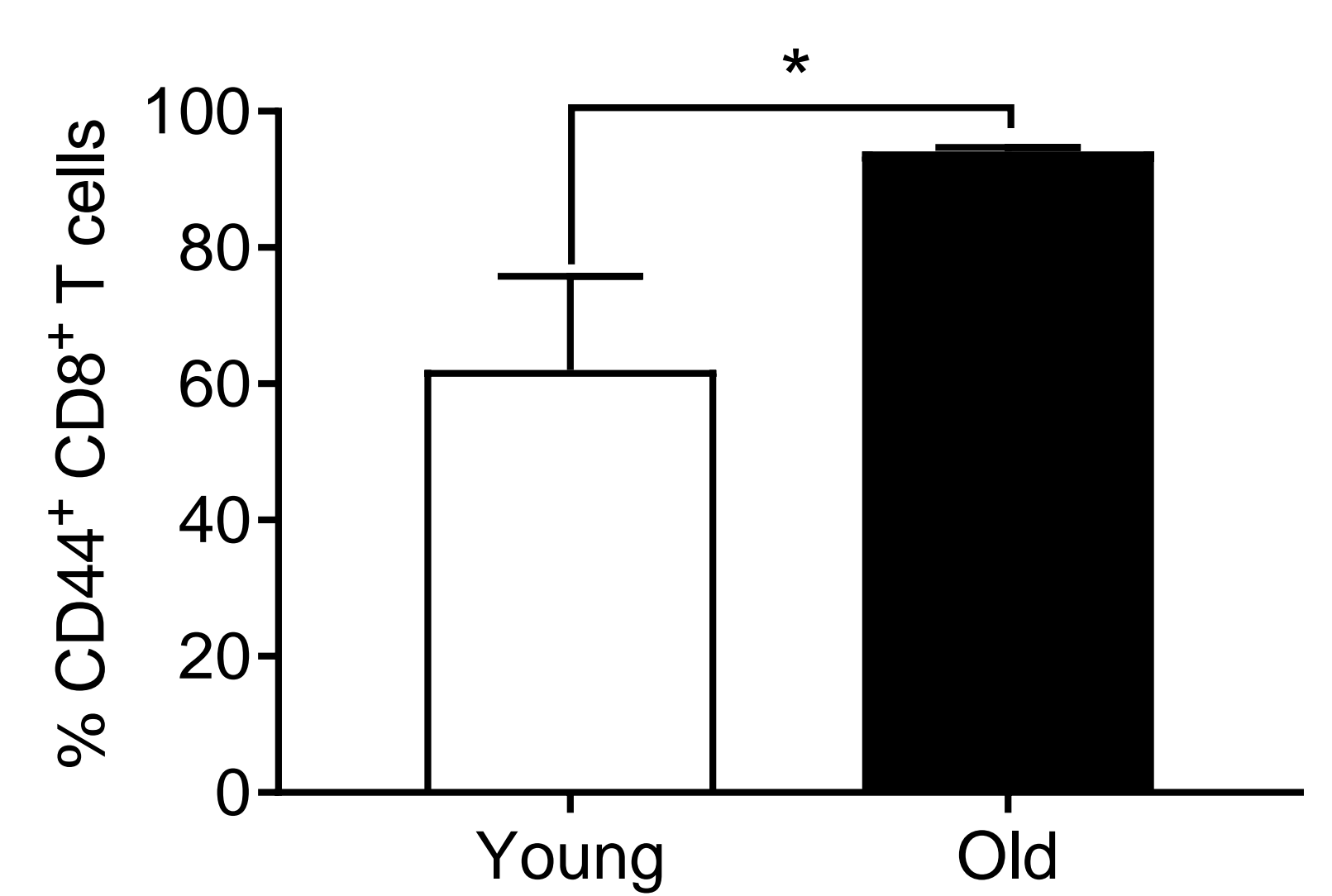

Memory_like T

Naive_T

CD8+EM_like T

CD4+EM_like T

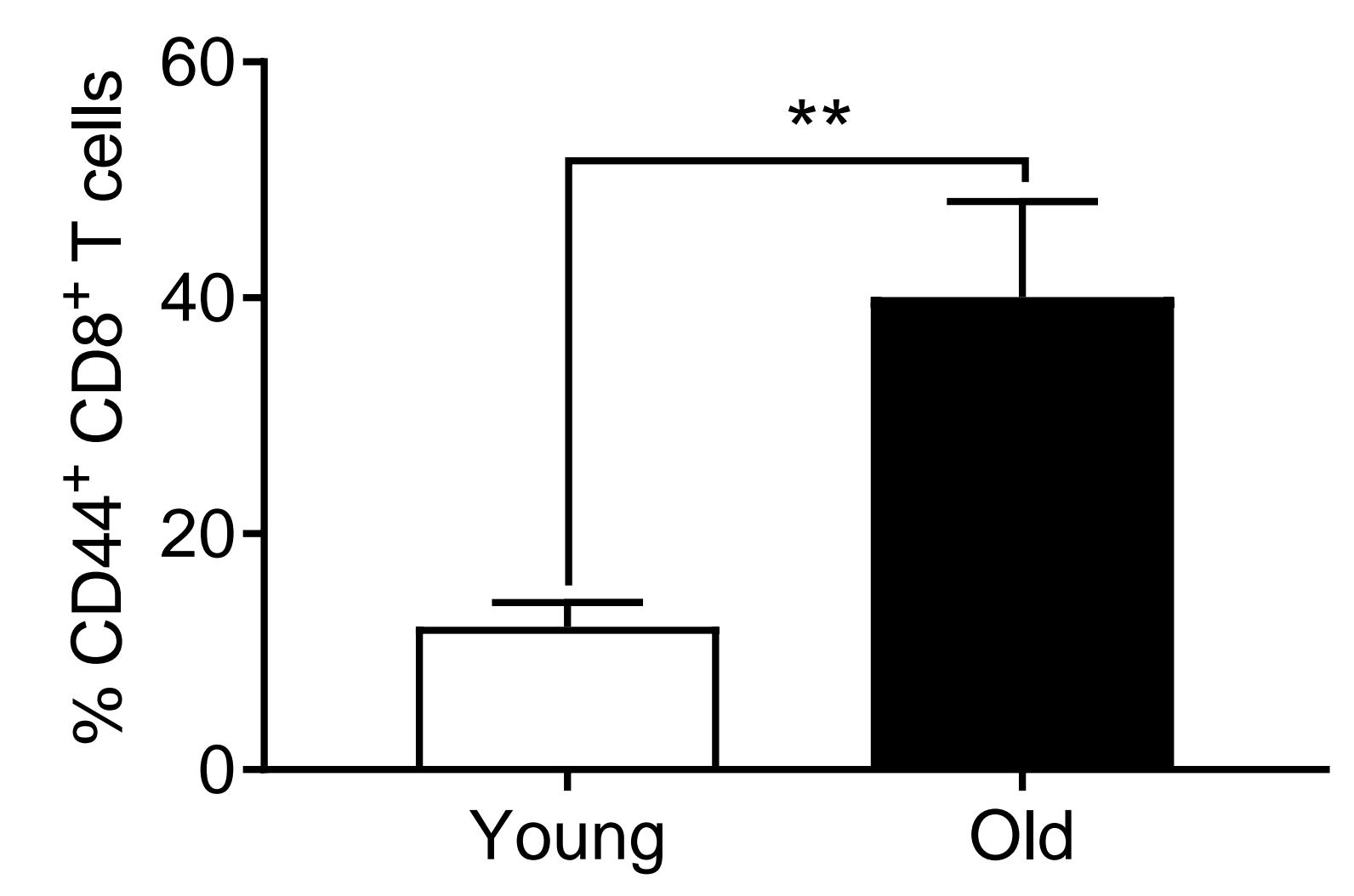

E
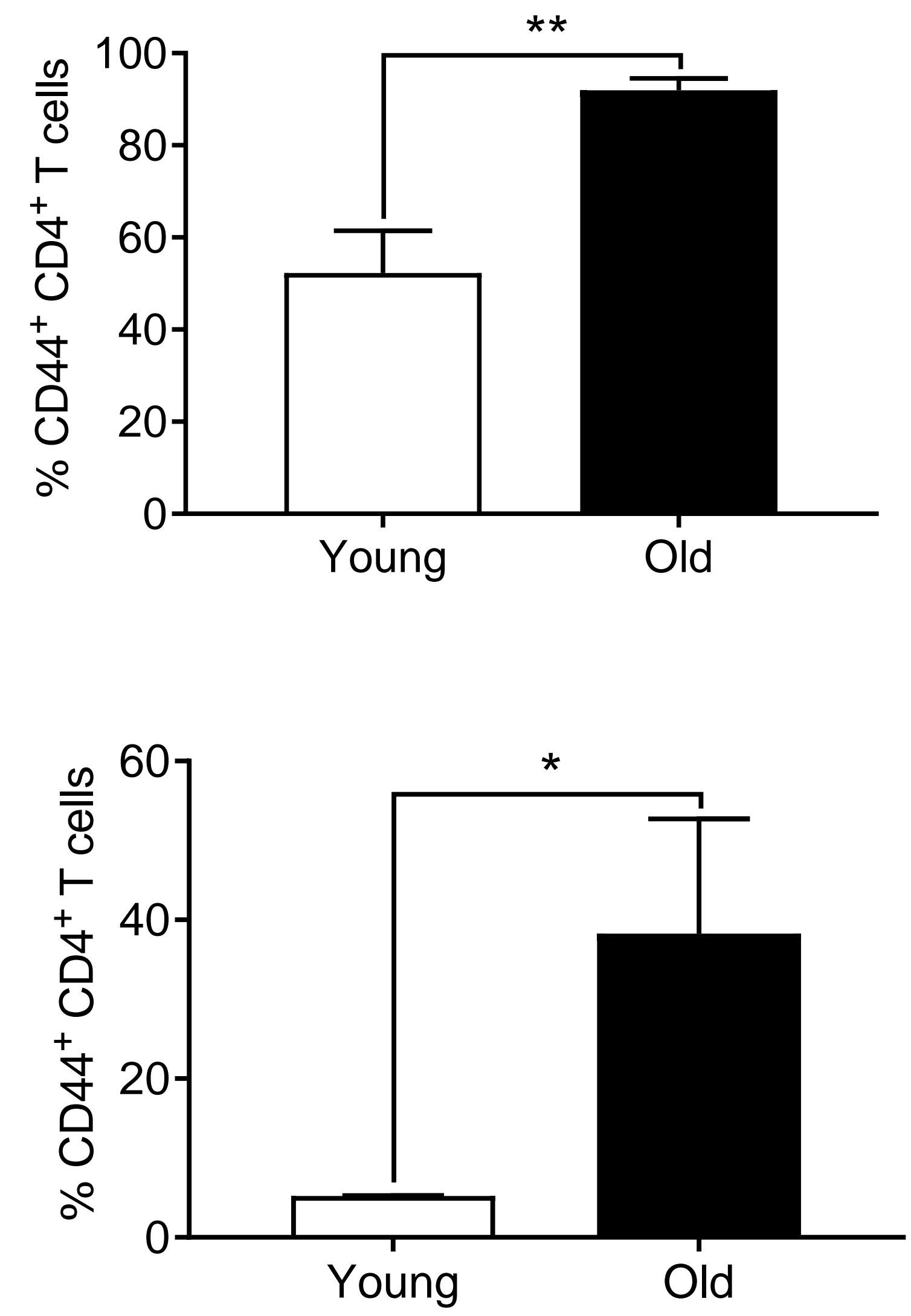

F

Young

Old
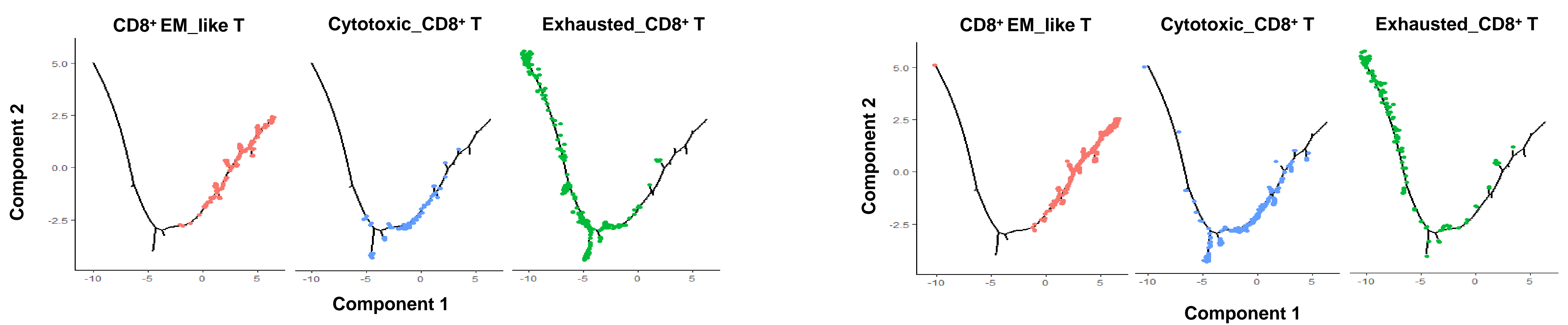
Supplementary Fig. 1

A

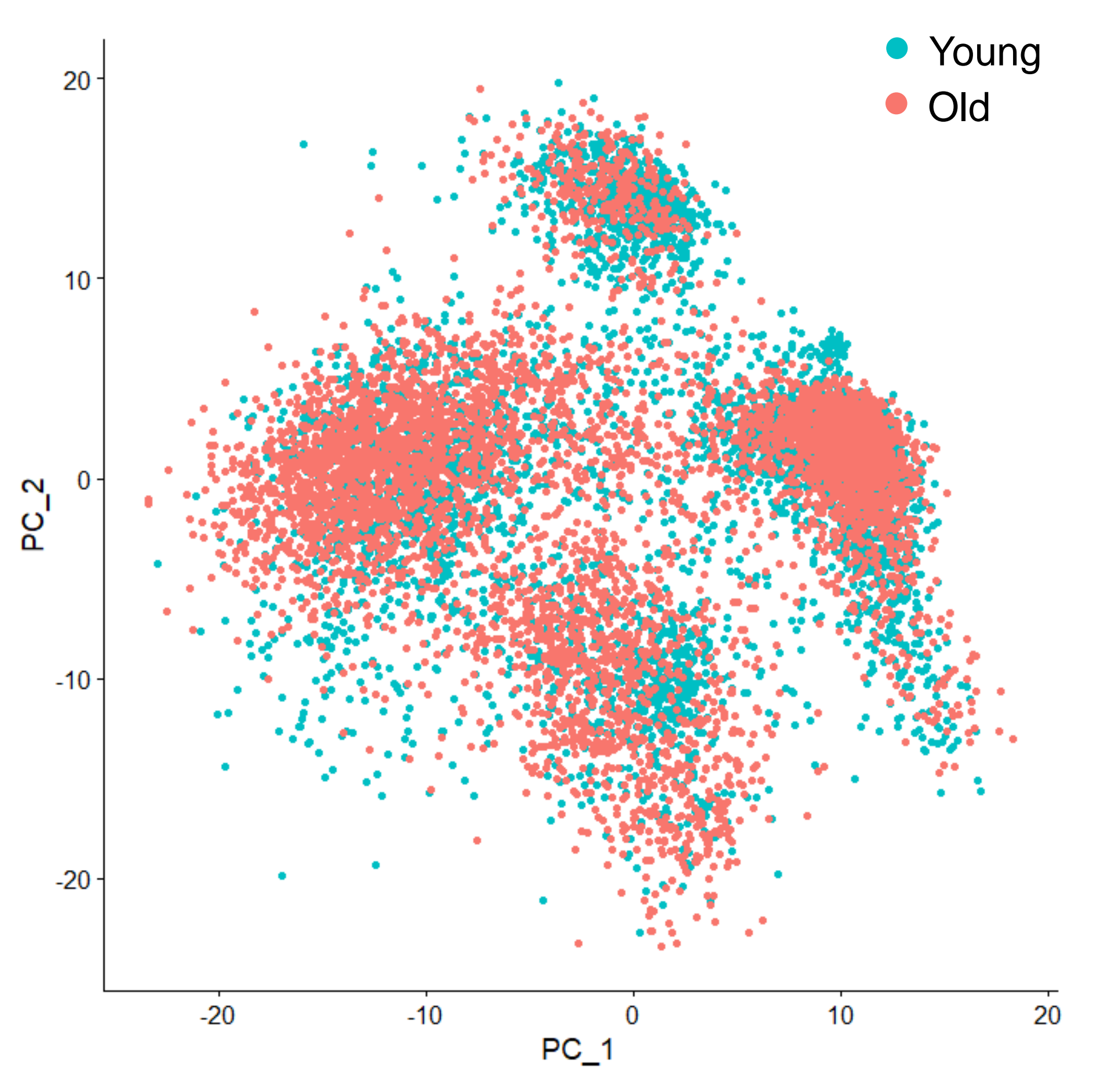

D

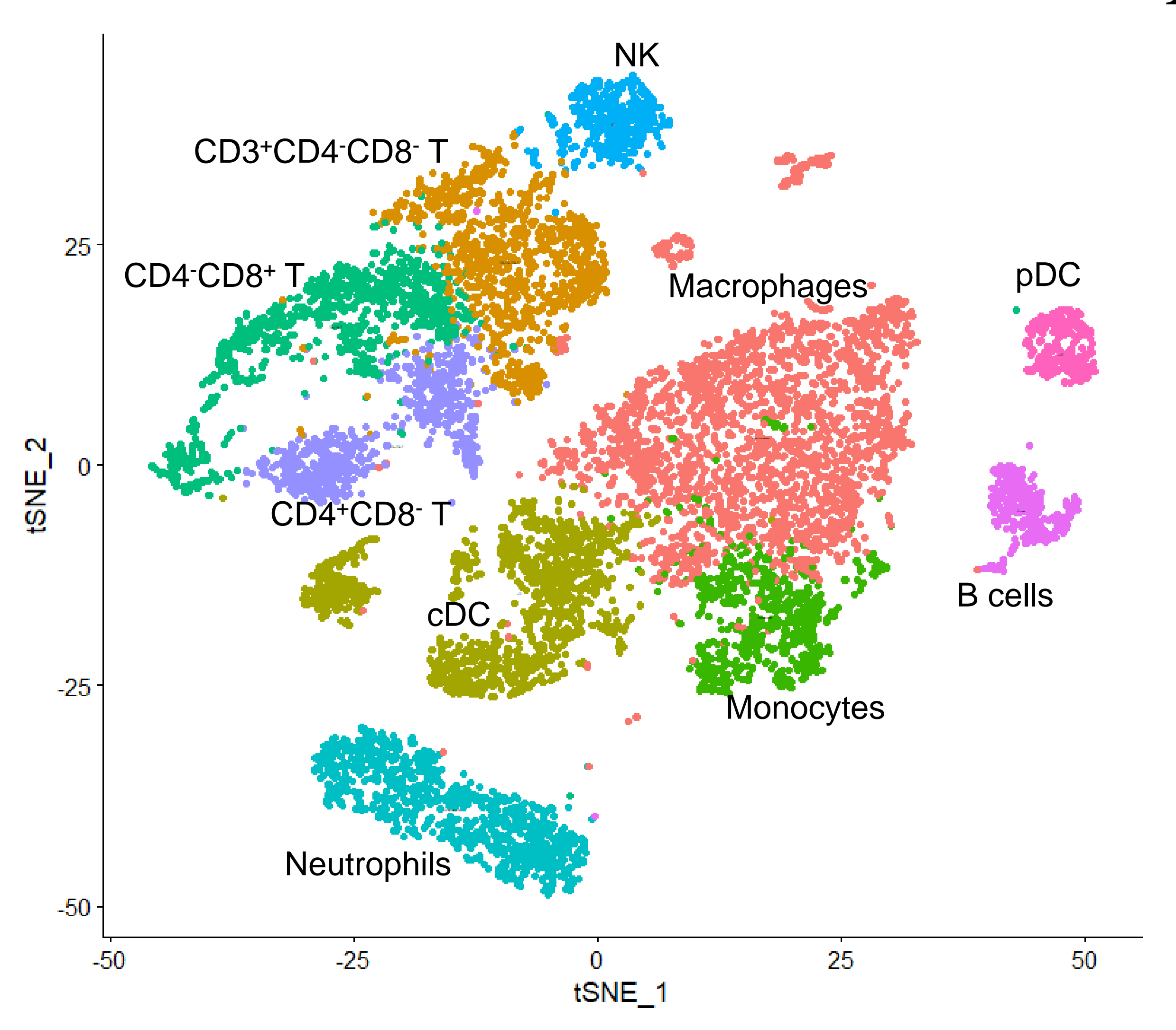

B

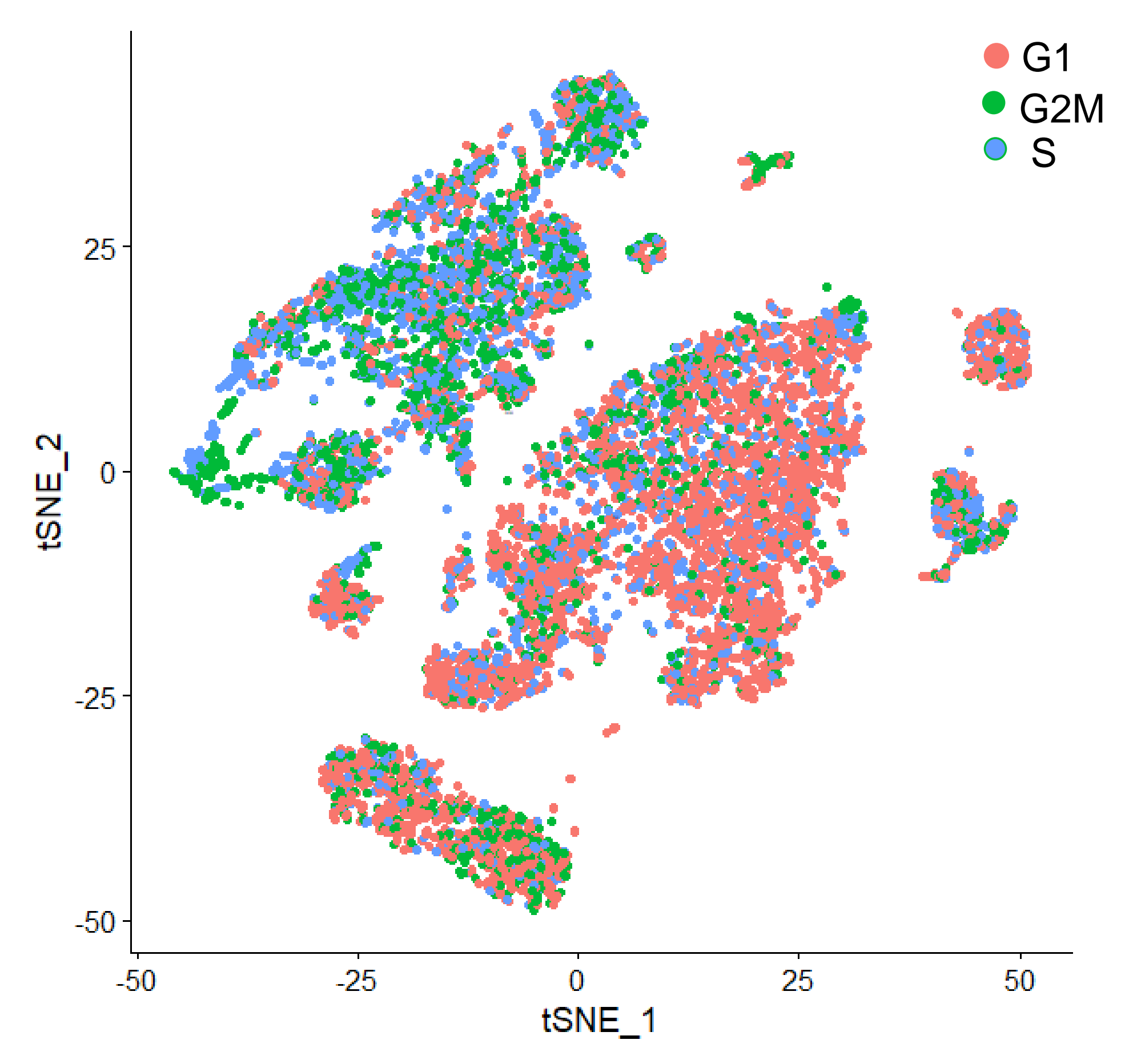

E

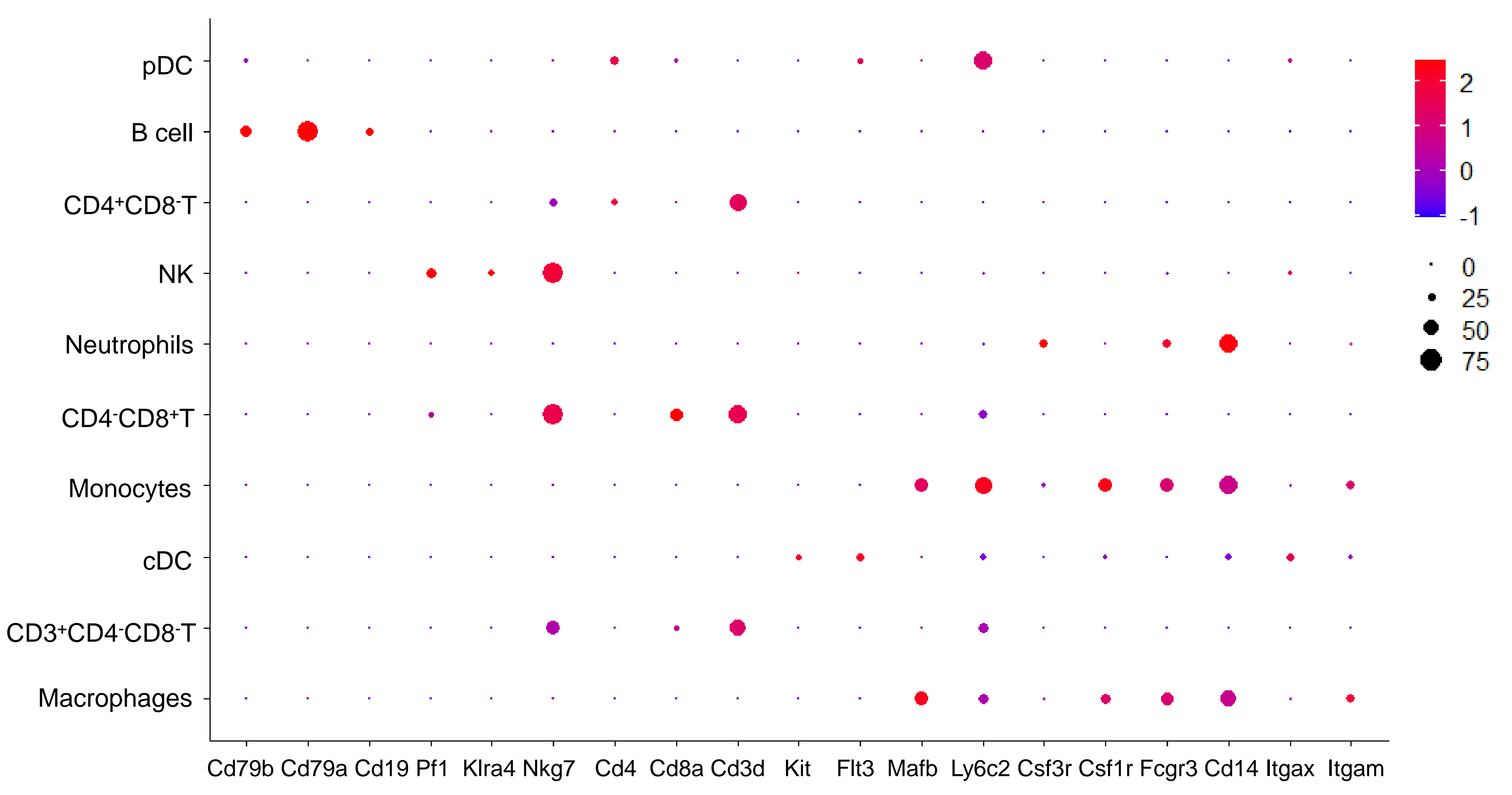

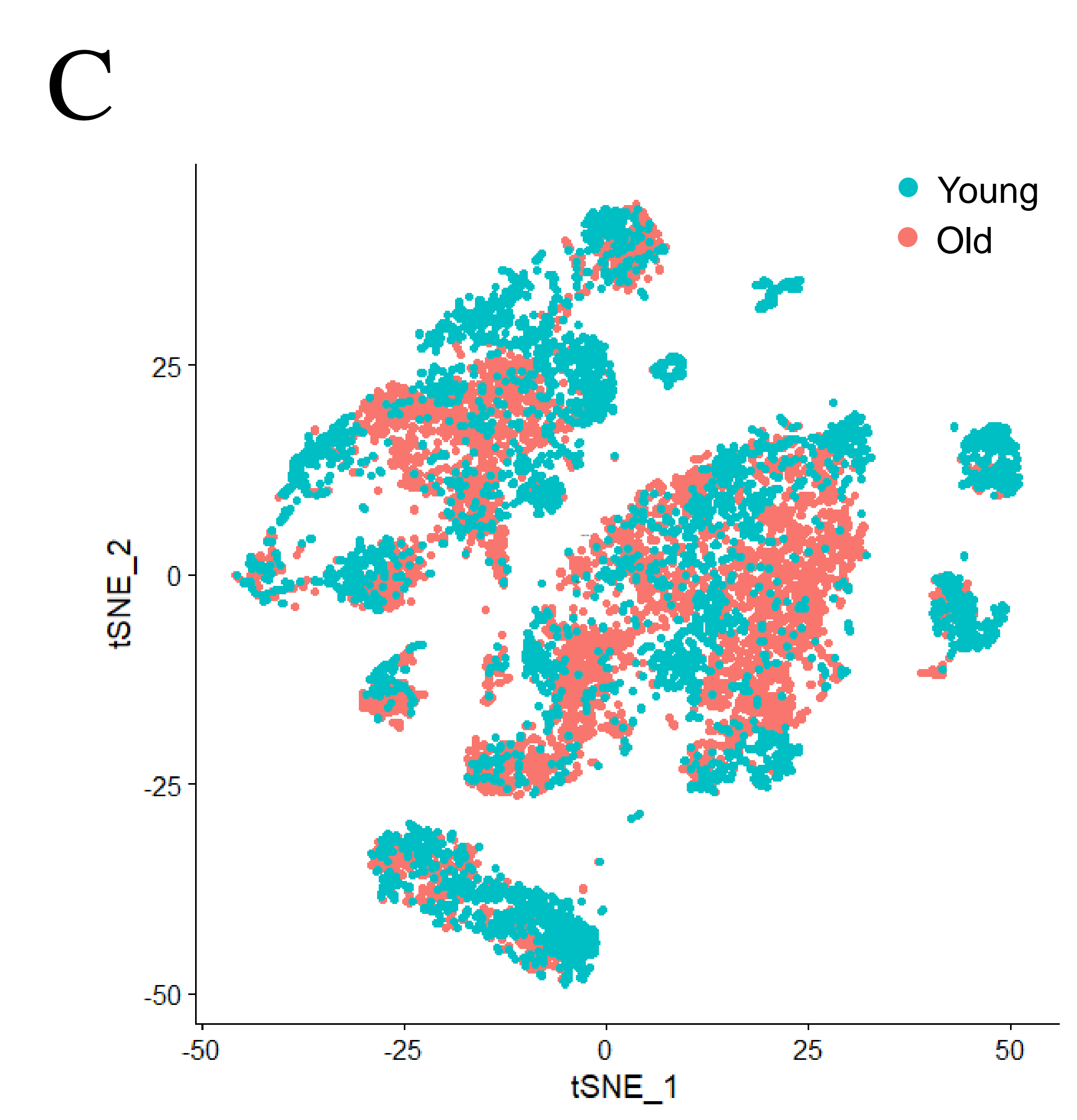


Supplementary Fig. 2
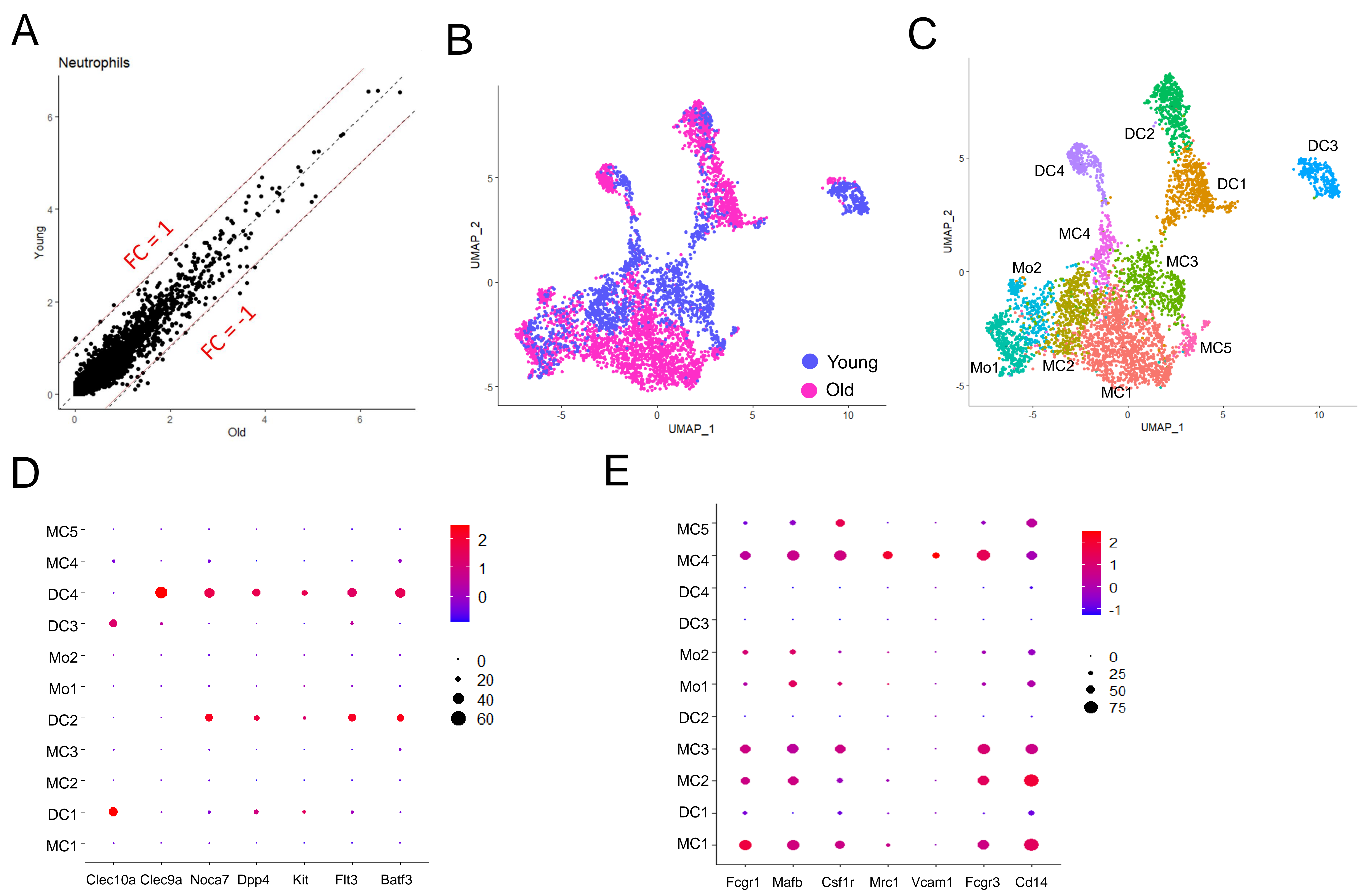

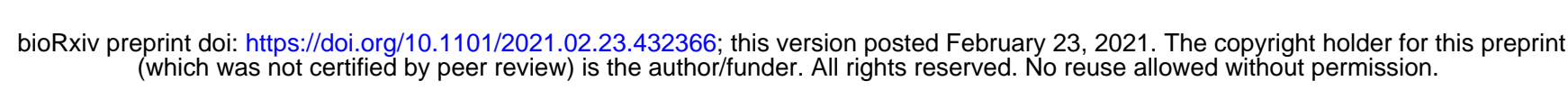


Supplementary Fig. 3

A B

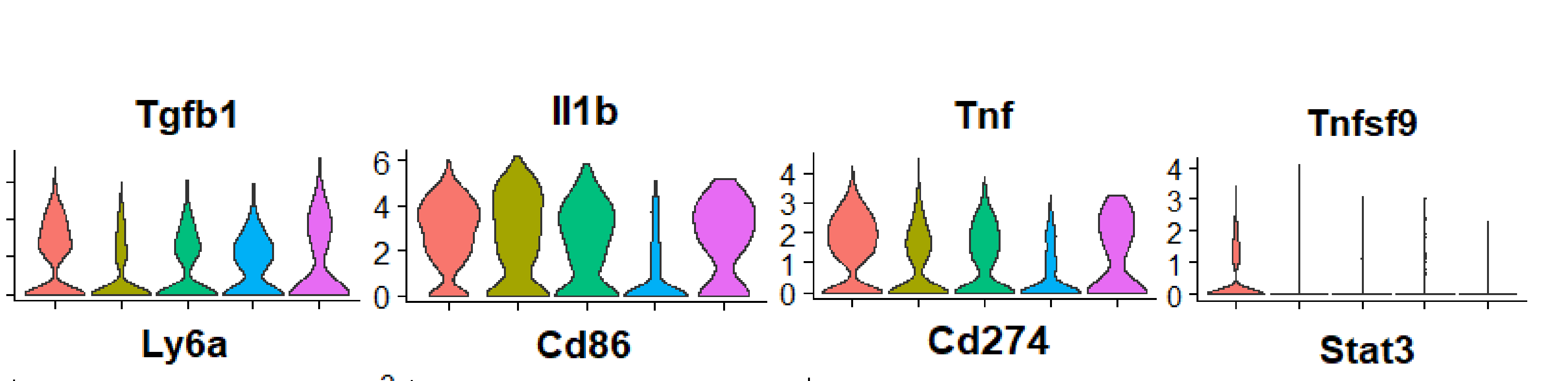

19.10 18

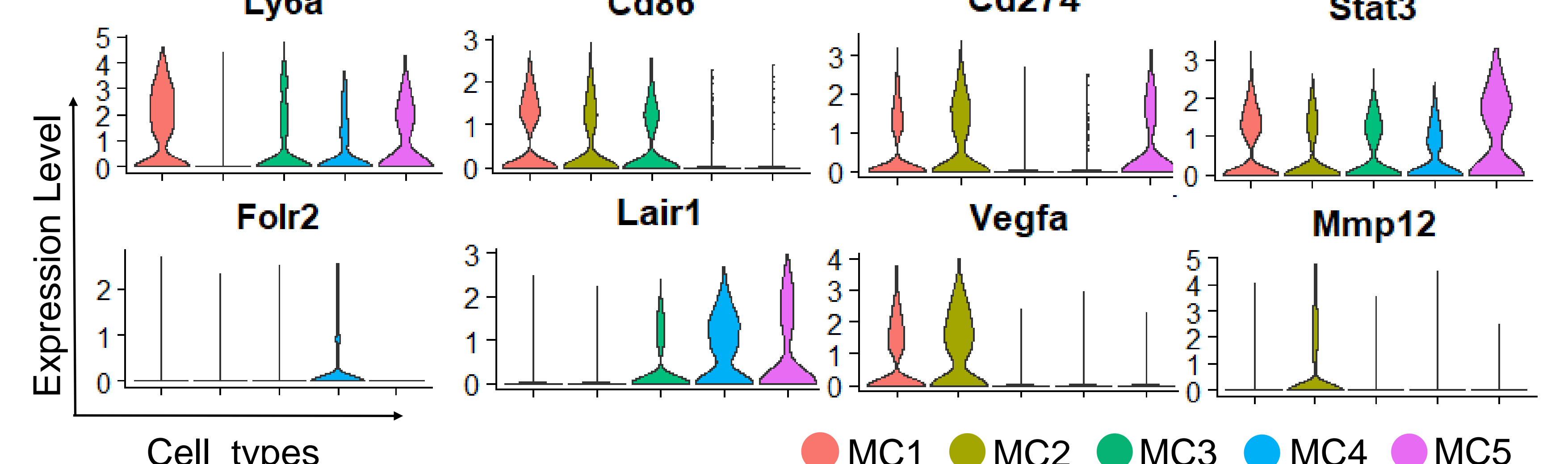

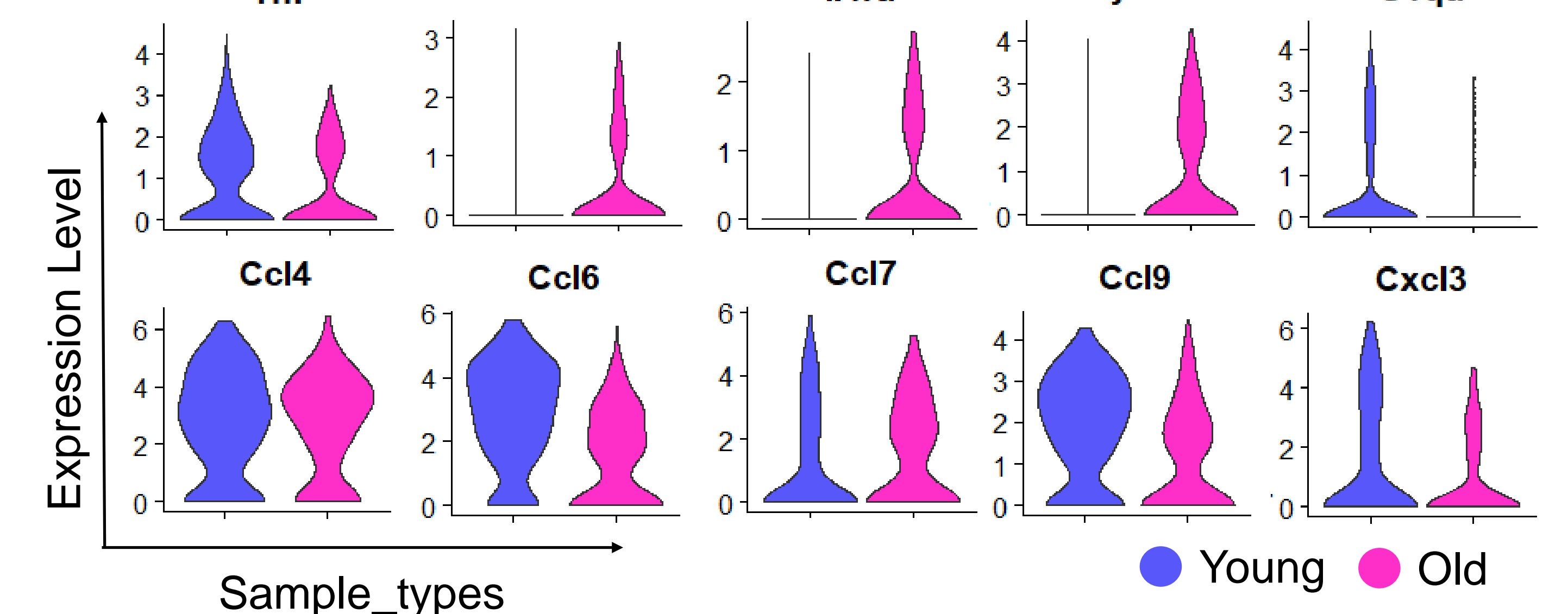
C

$$
\begin{aligned}
& \text { IILIITI? } \\
& 11.1111
\end{aligned}
$$


Supplementary Fig. 4

A

B

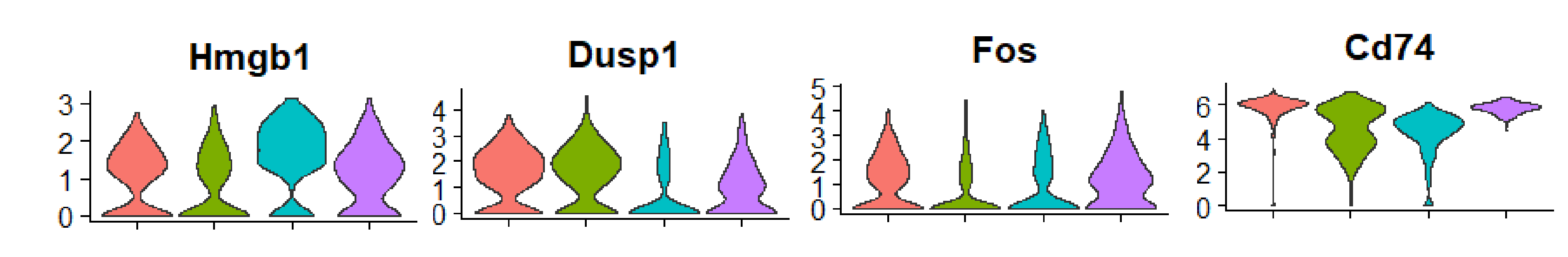
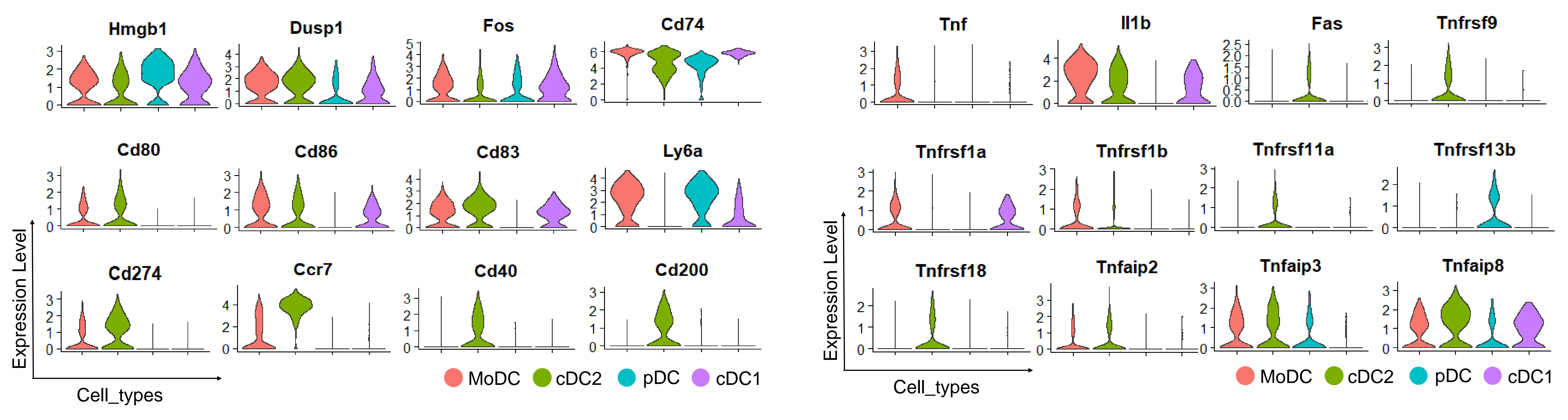

C

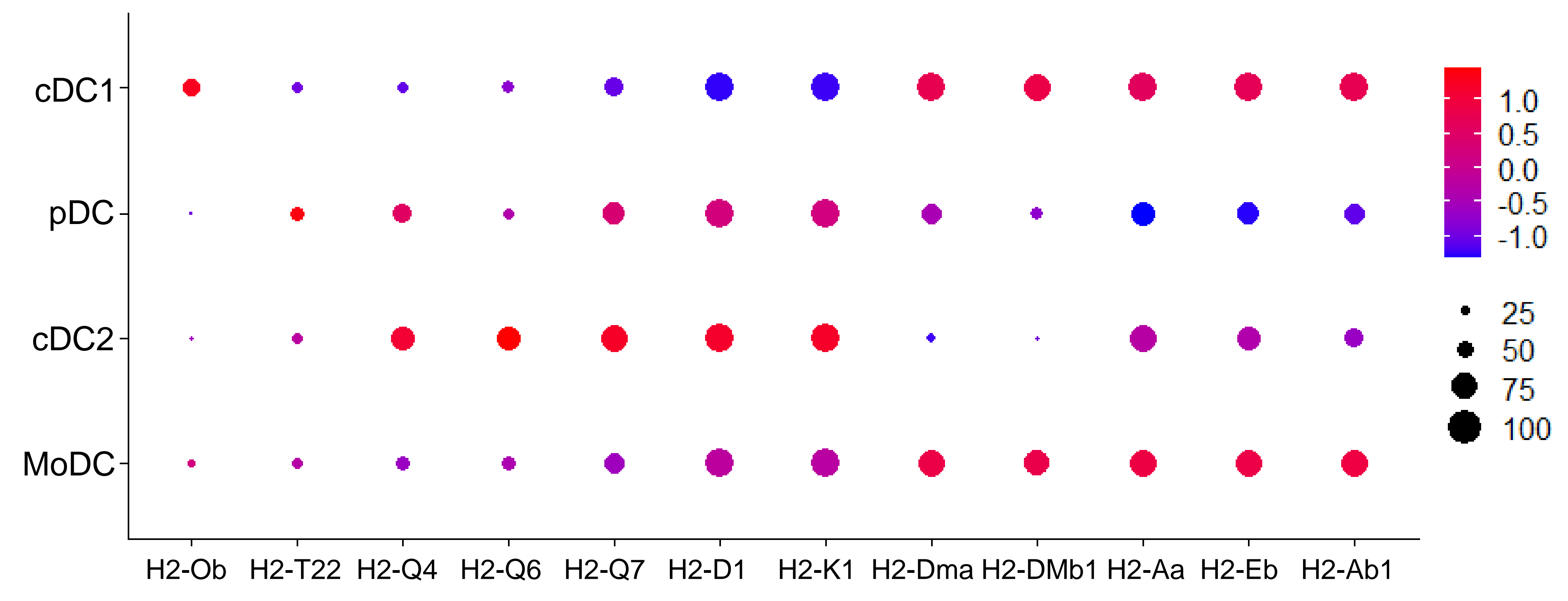


A
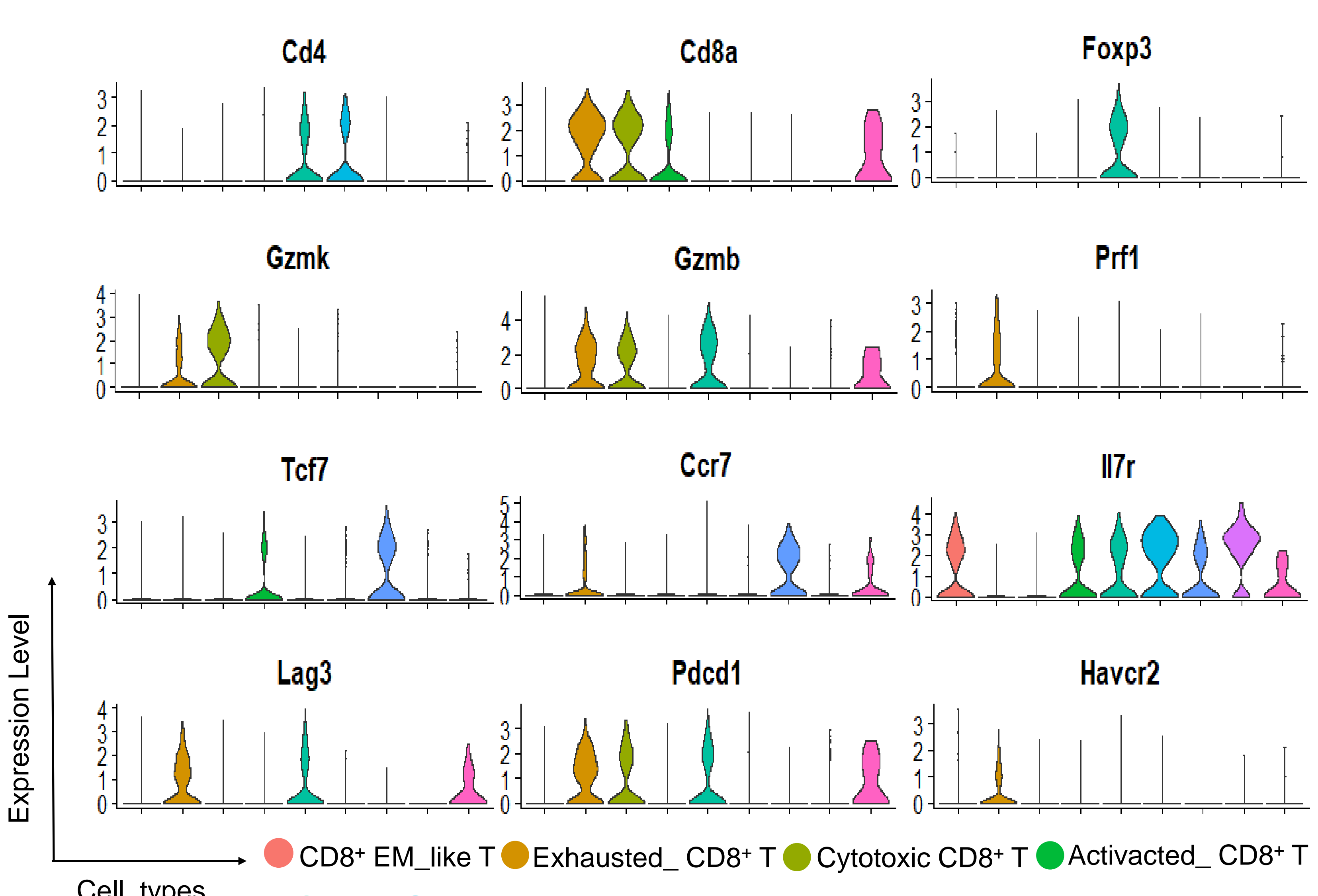
Cell_types

B
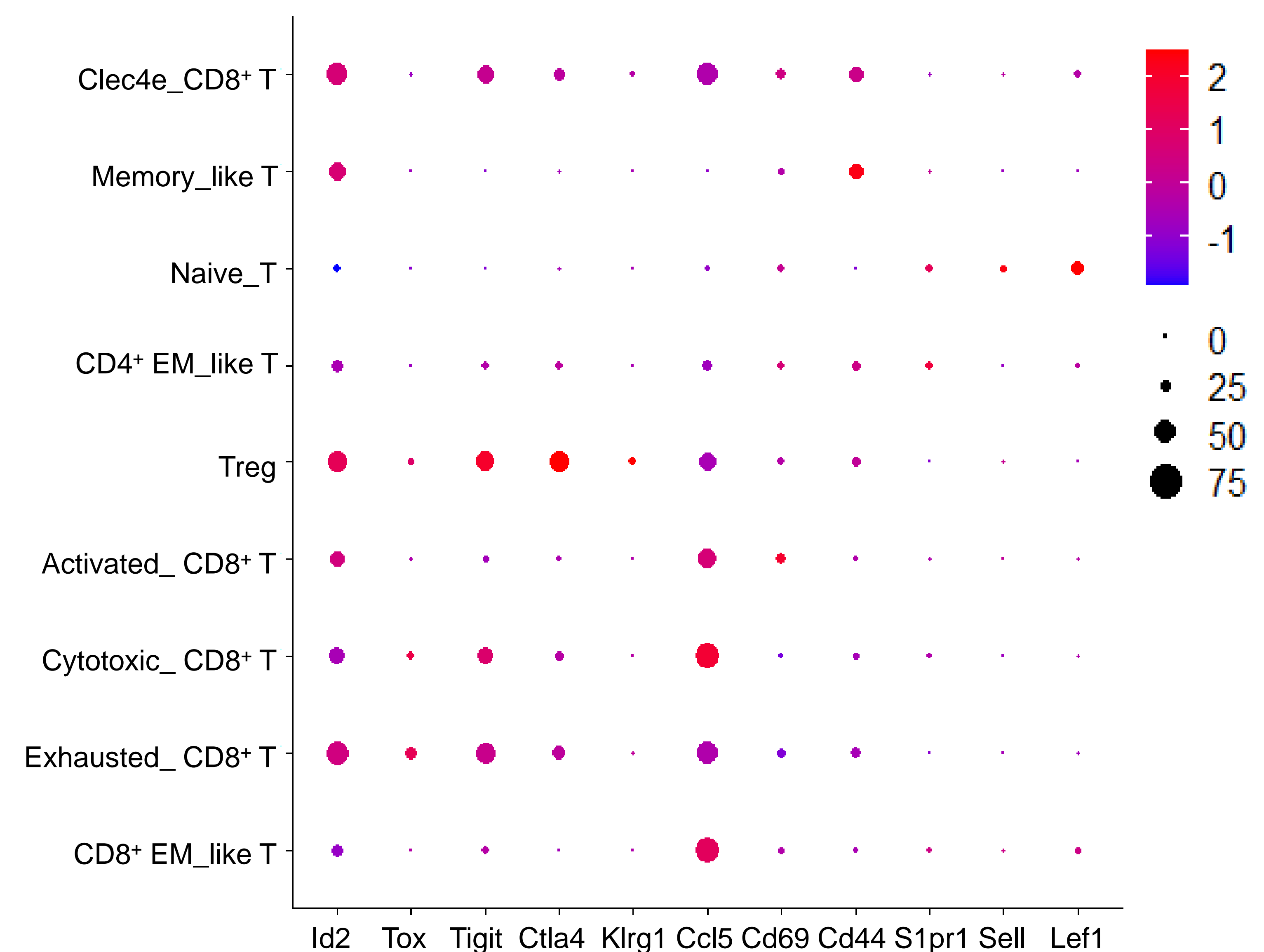

C

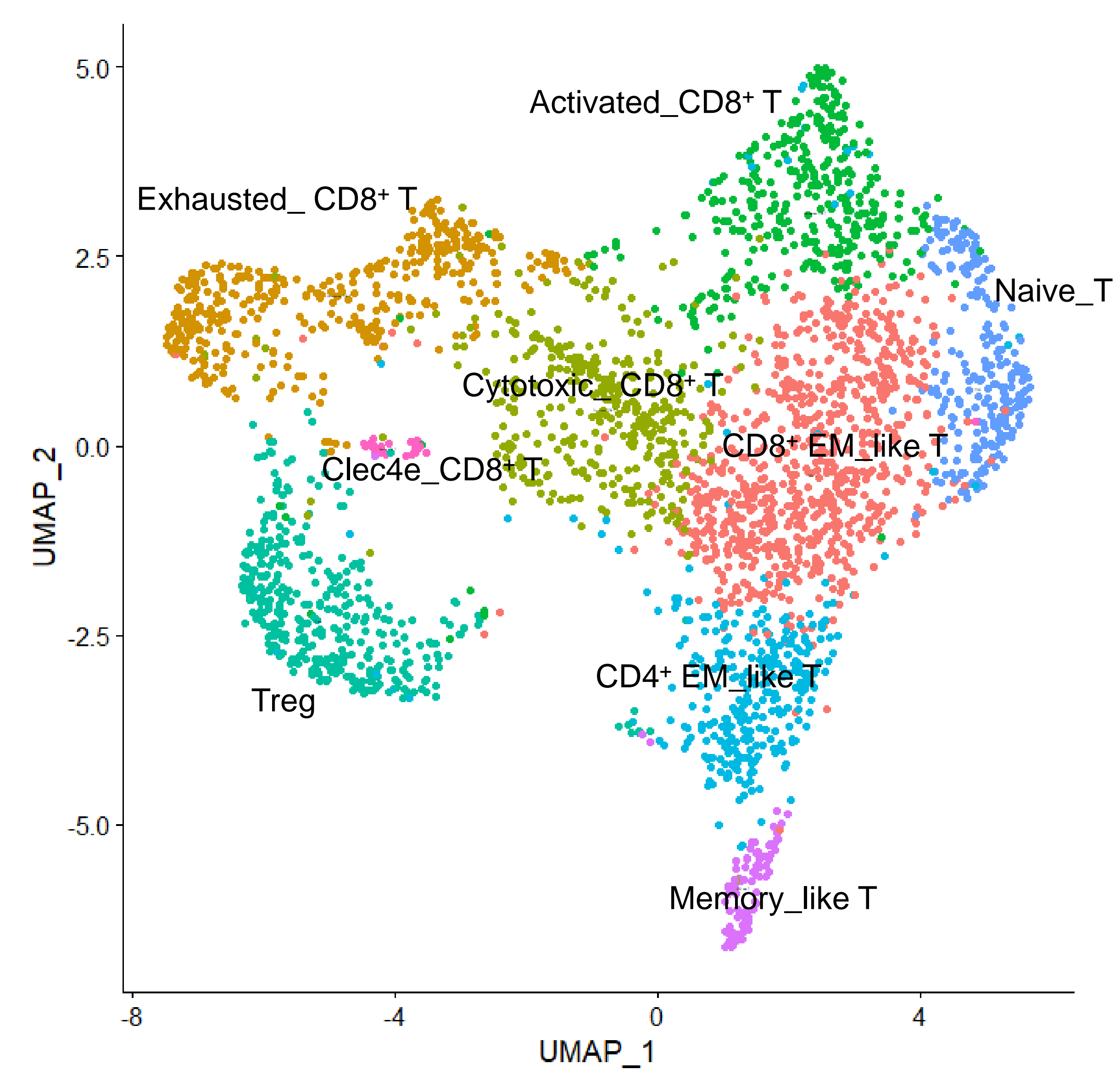


A

INDUCED_VS_NATURAL_TREG_DN
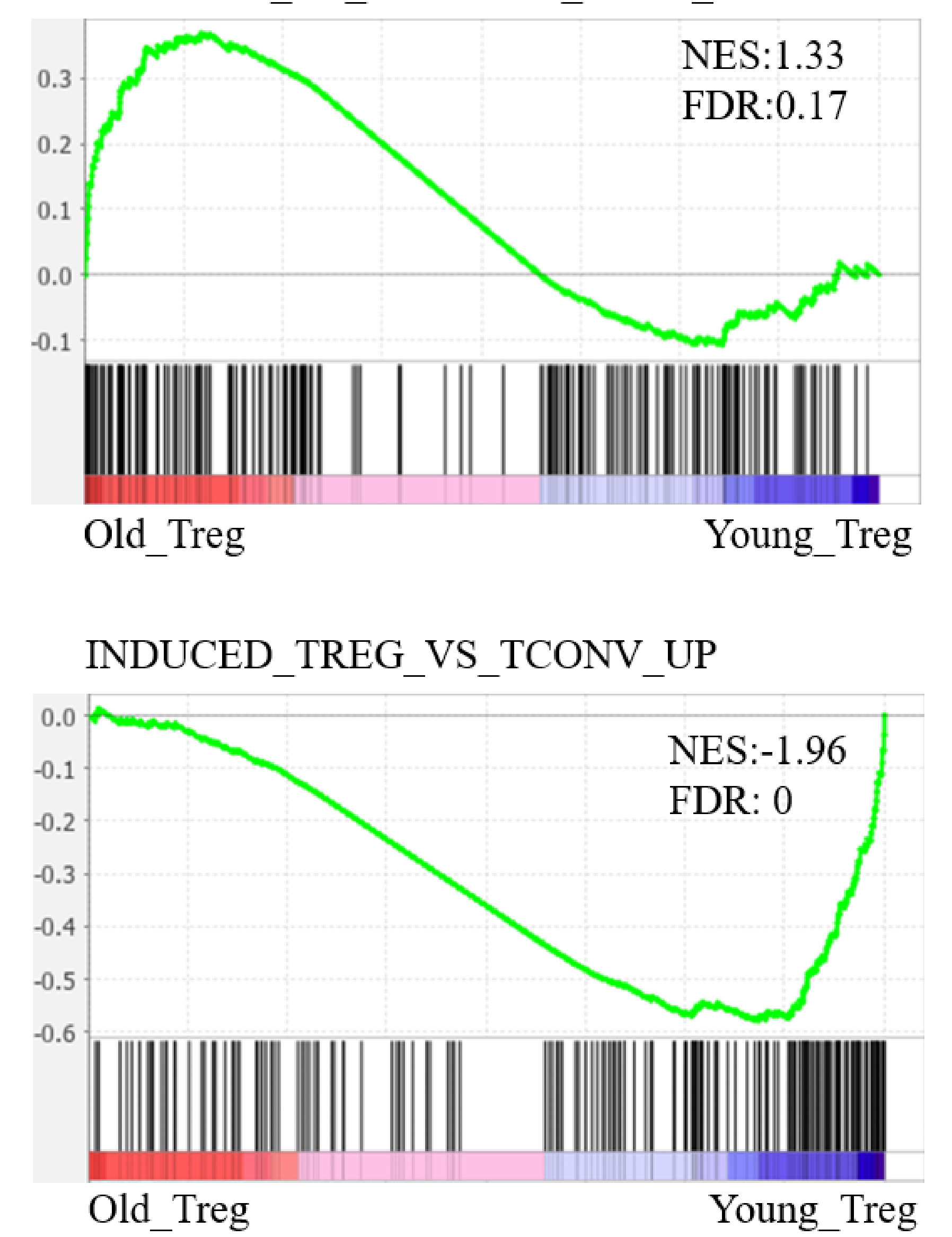

B

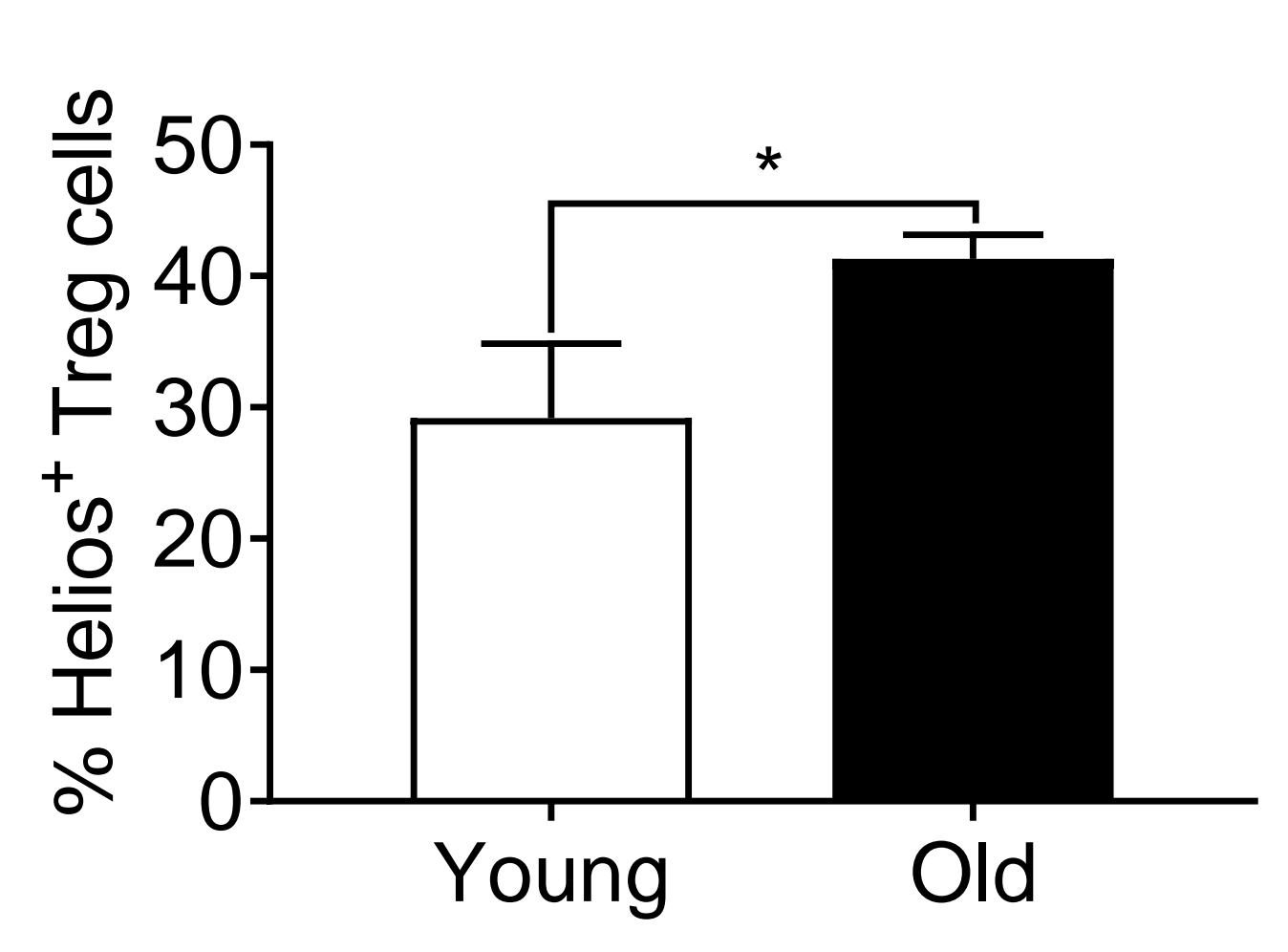

C

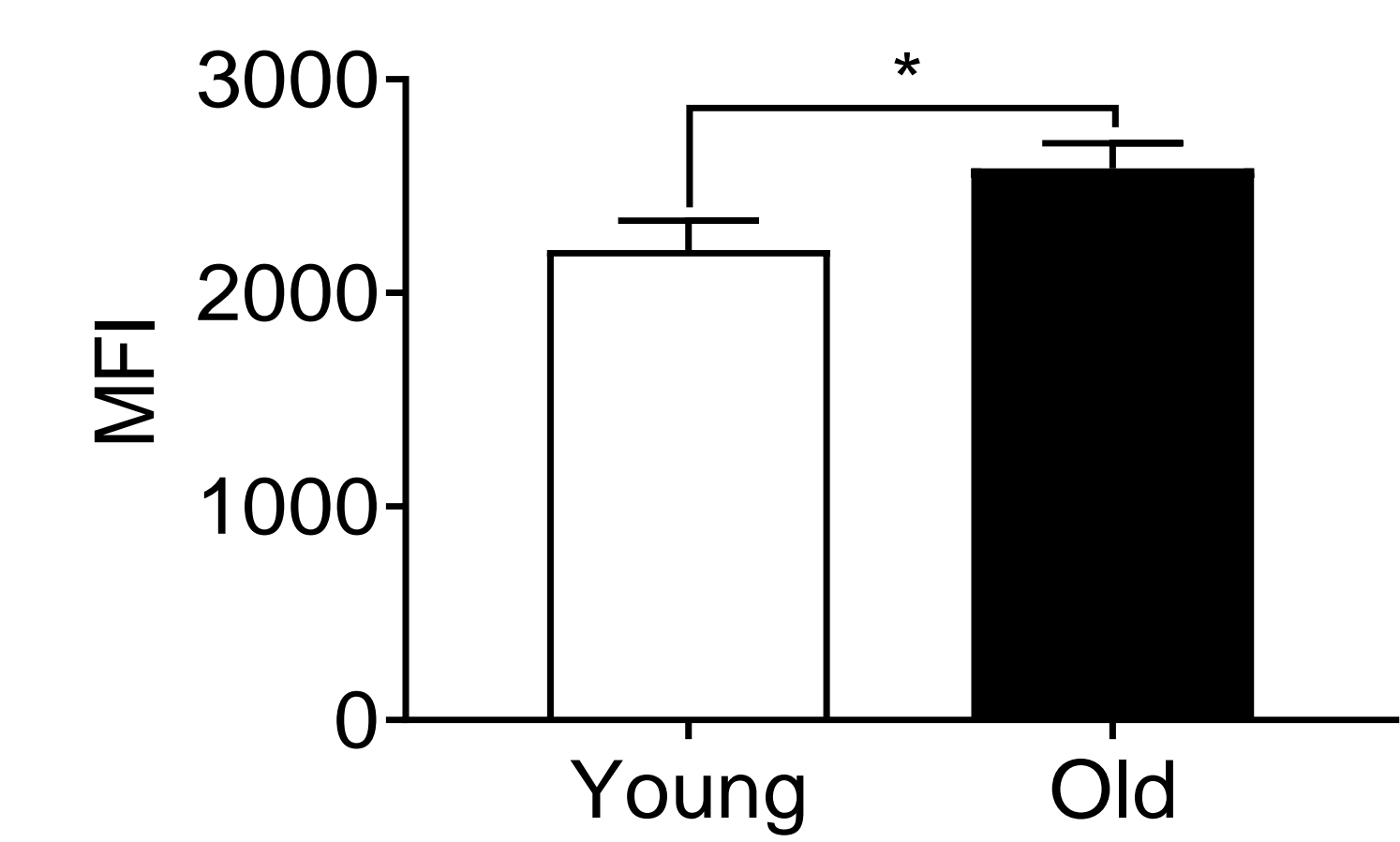

D
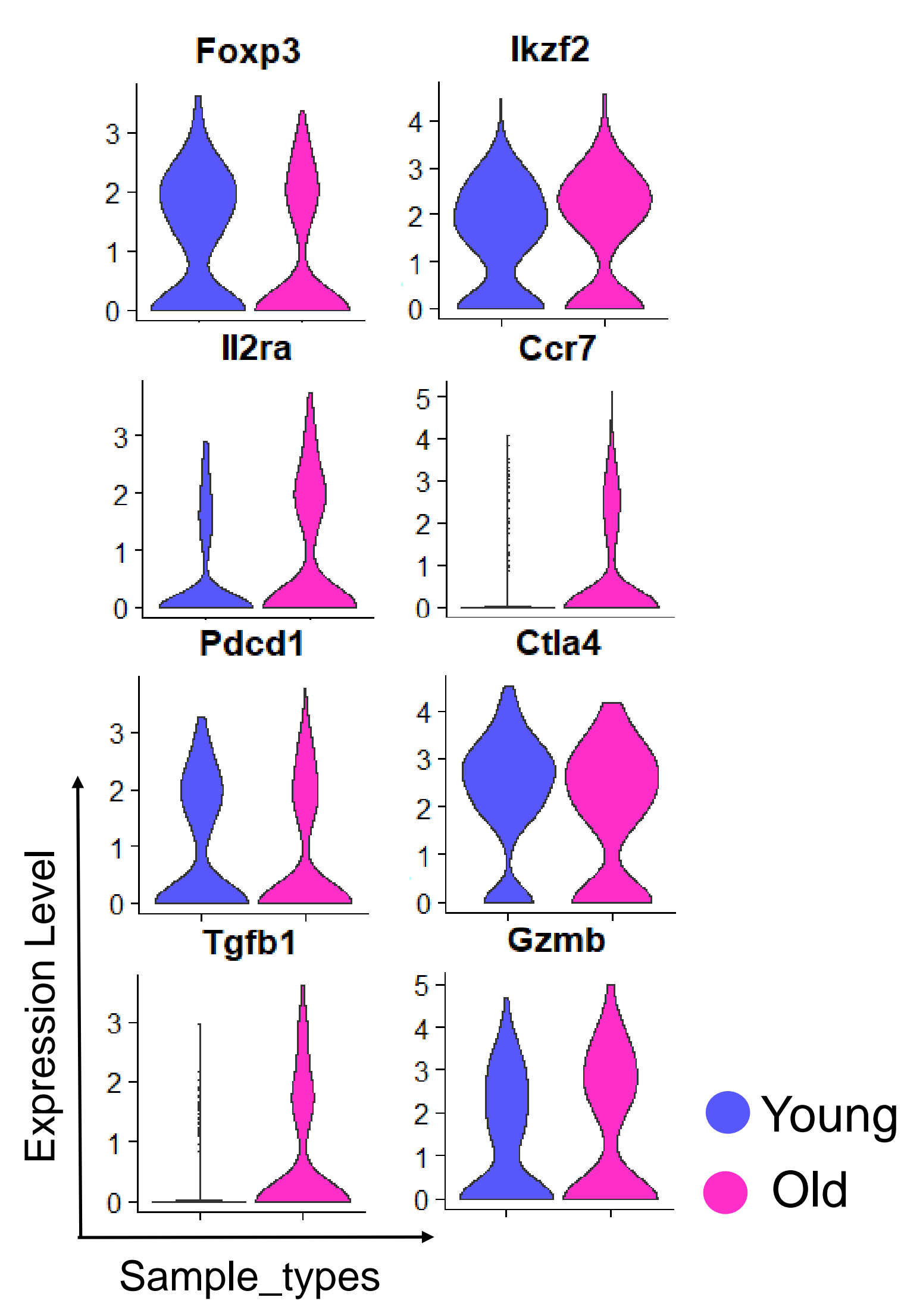

E

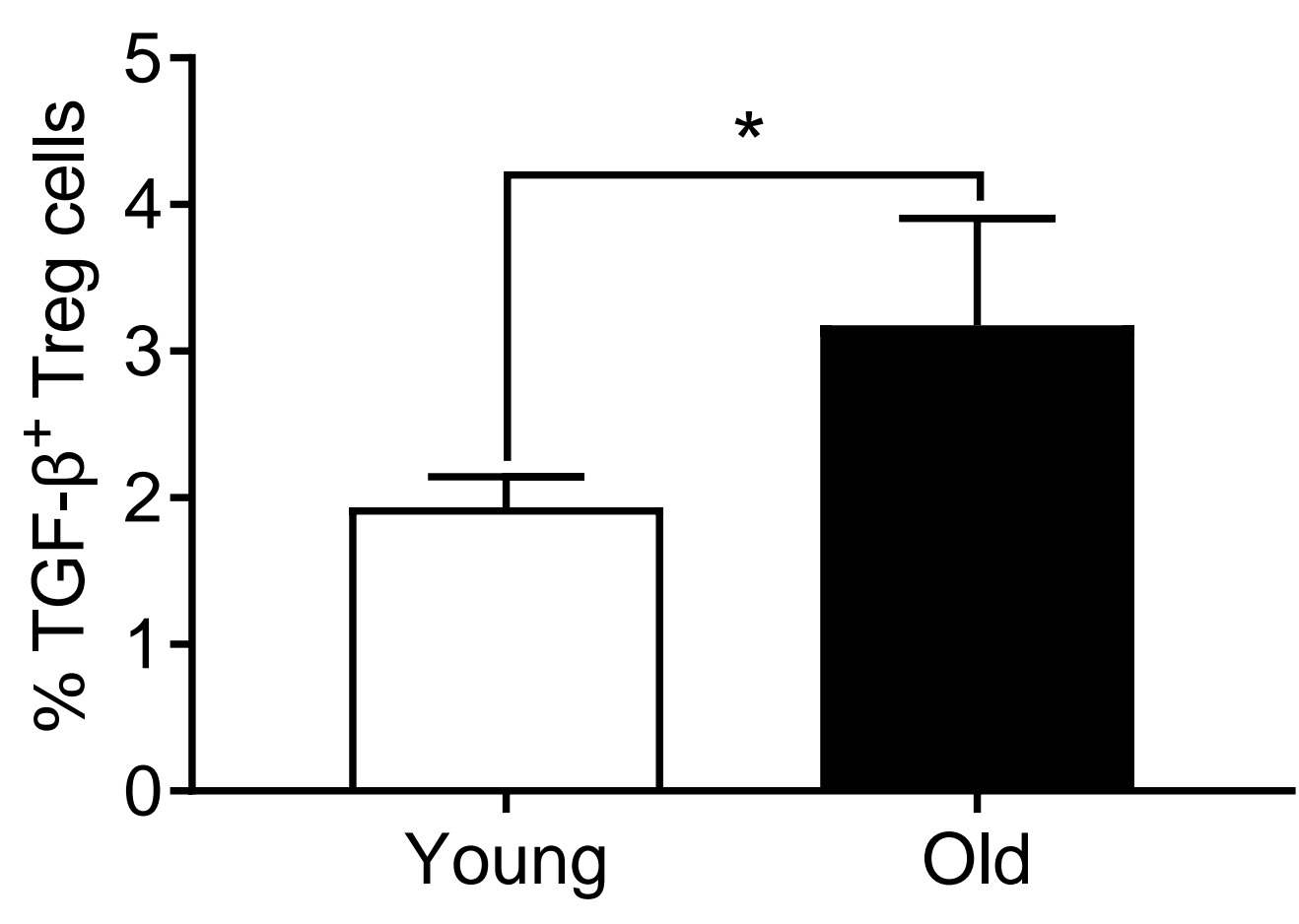

$\mathrm{F}$

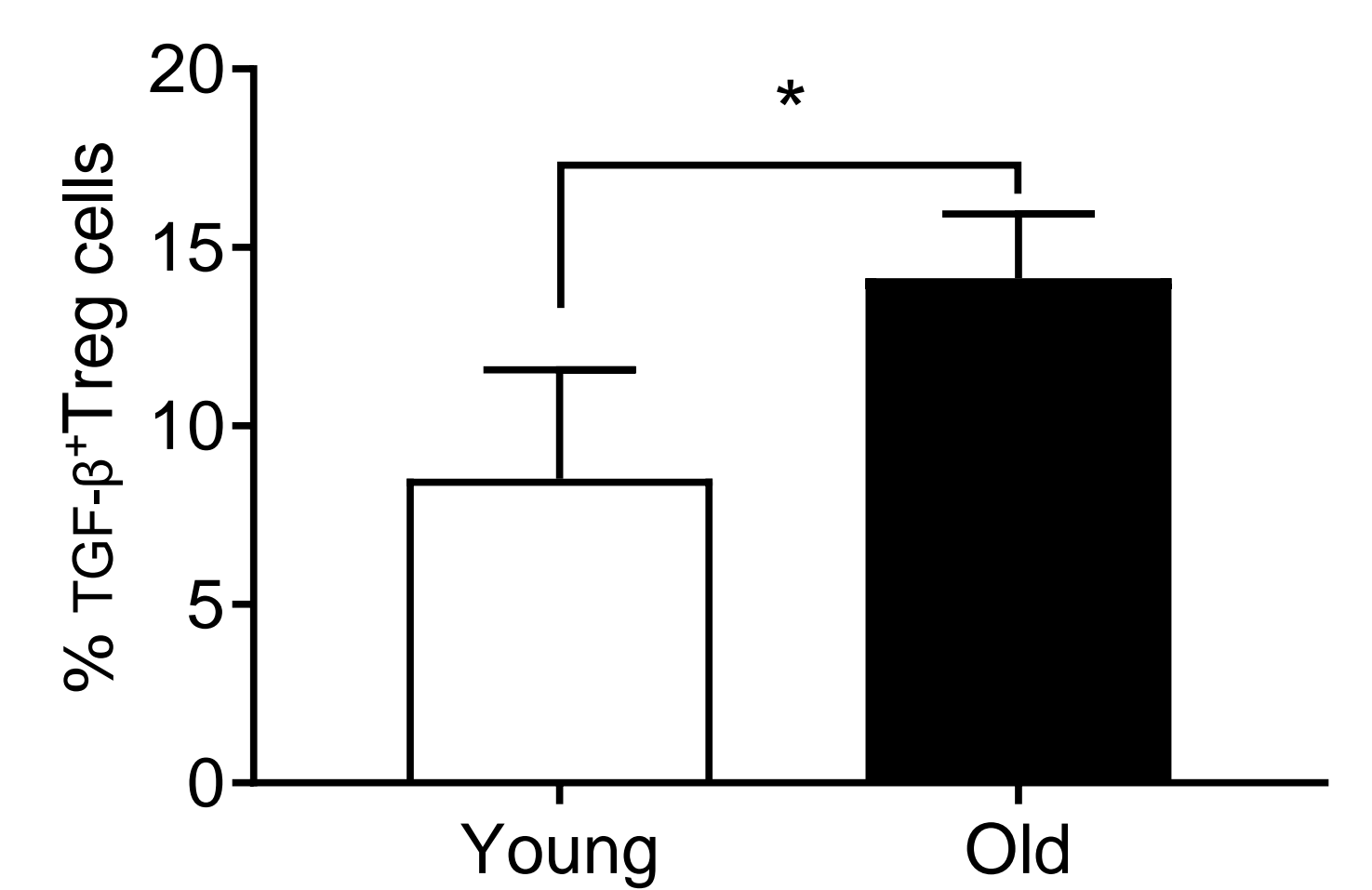


A

B
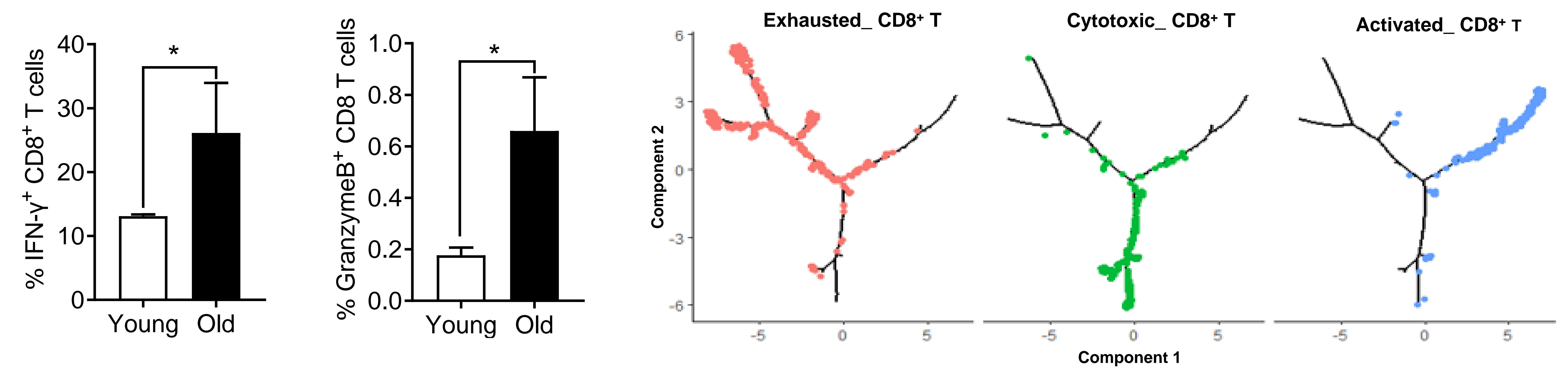

C

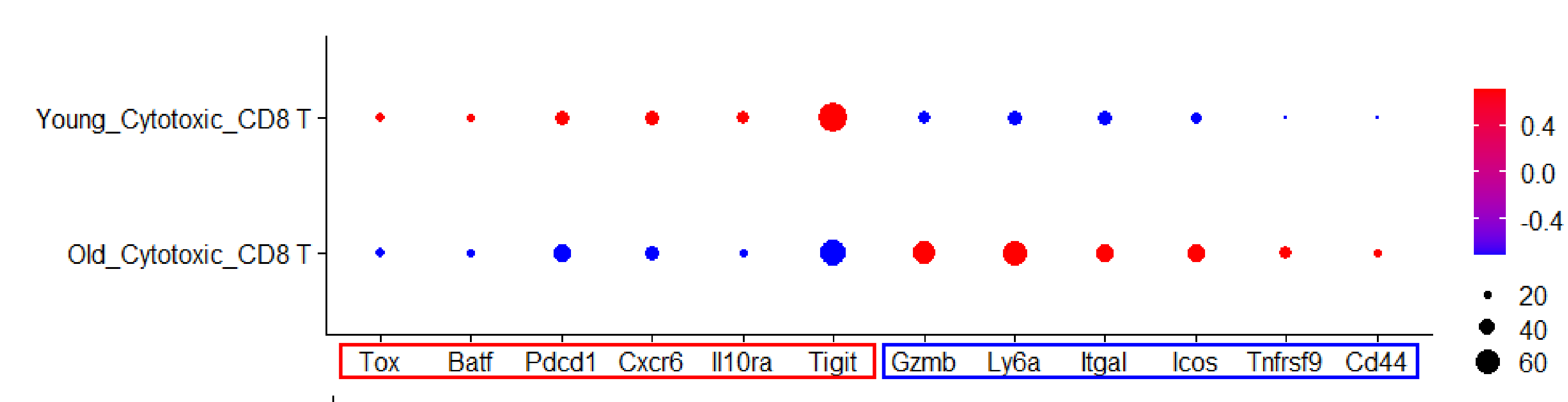

D
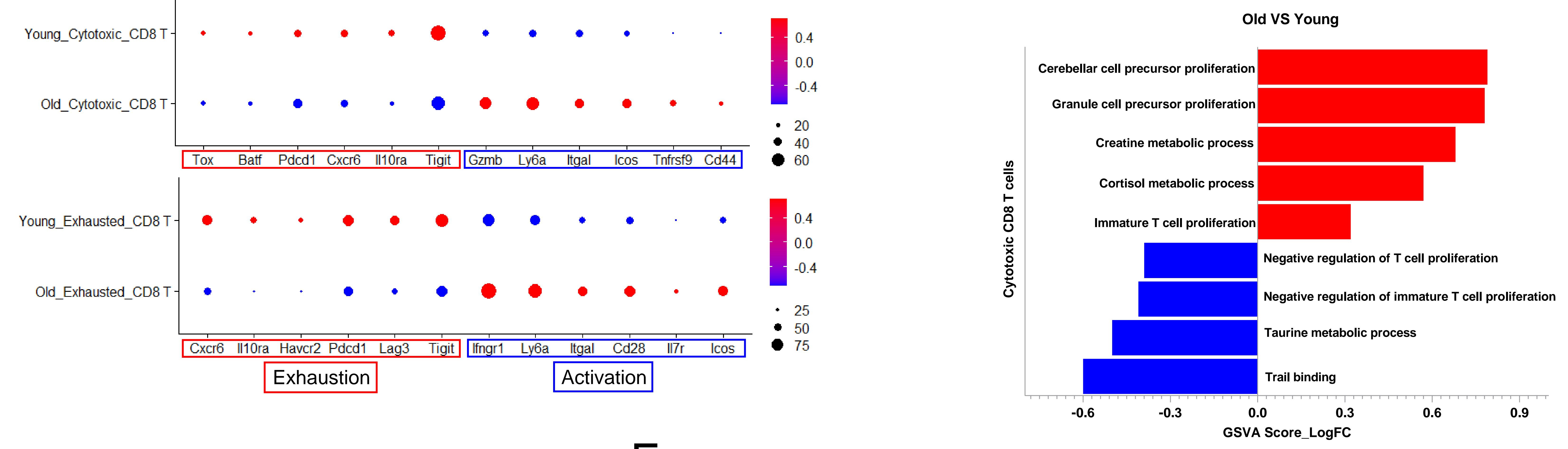

$E$

F
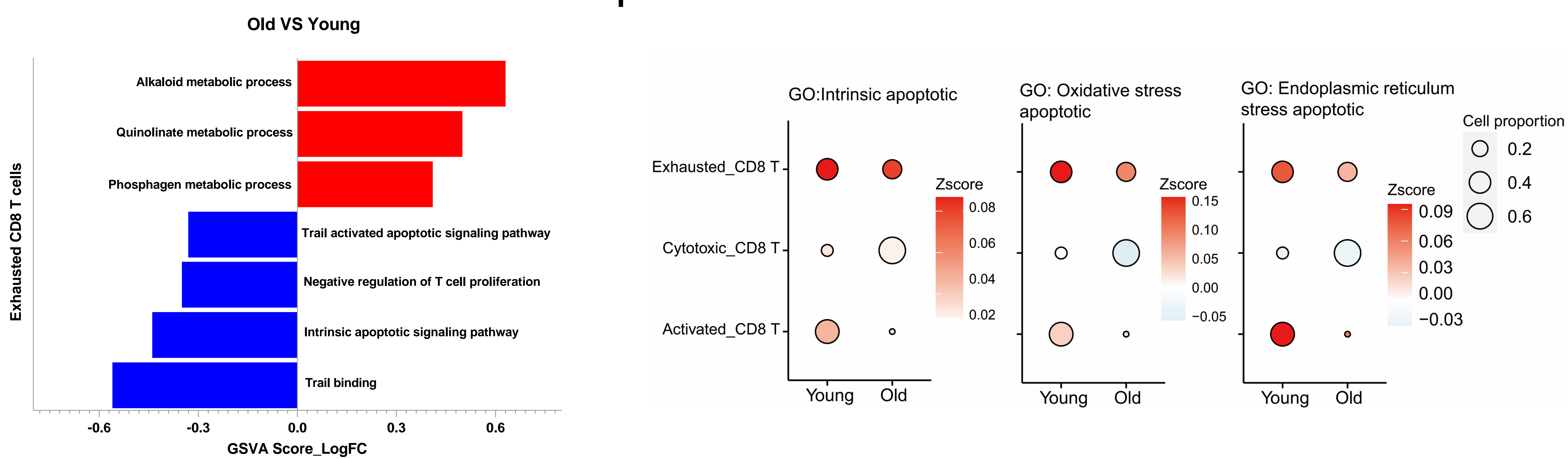
Supplementary Fig. 8

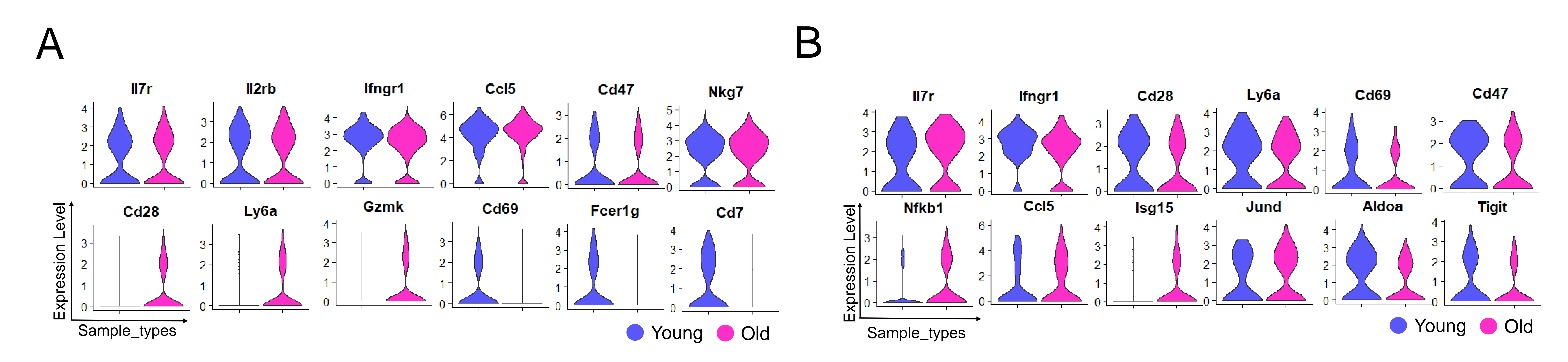

C

D
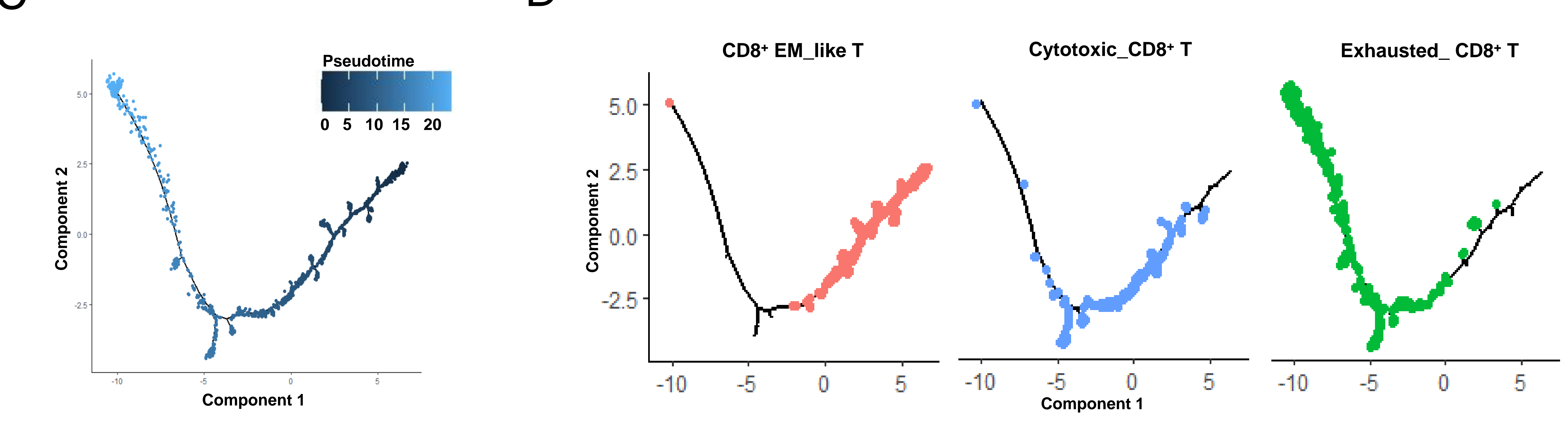
A

LIN_NEG_VS_NKCELL_DN
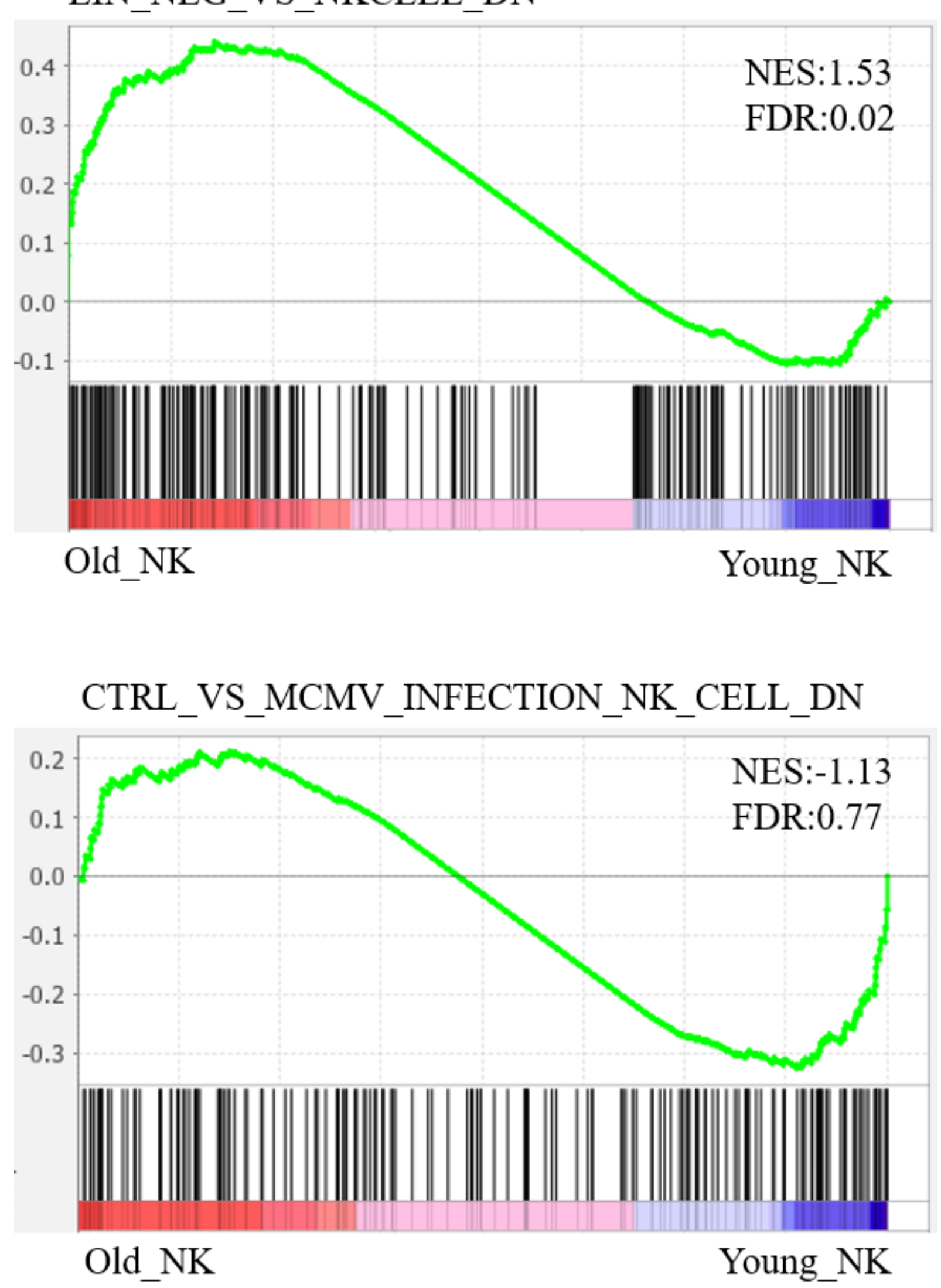

B

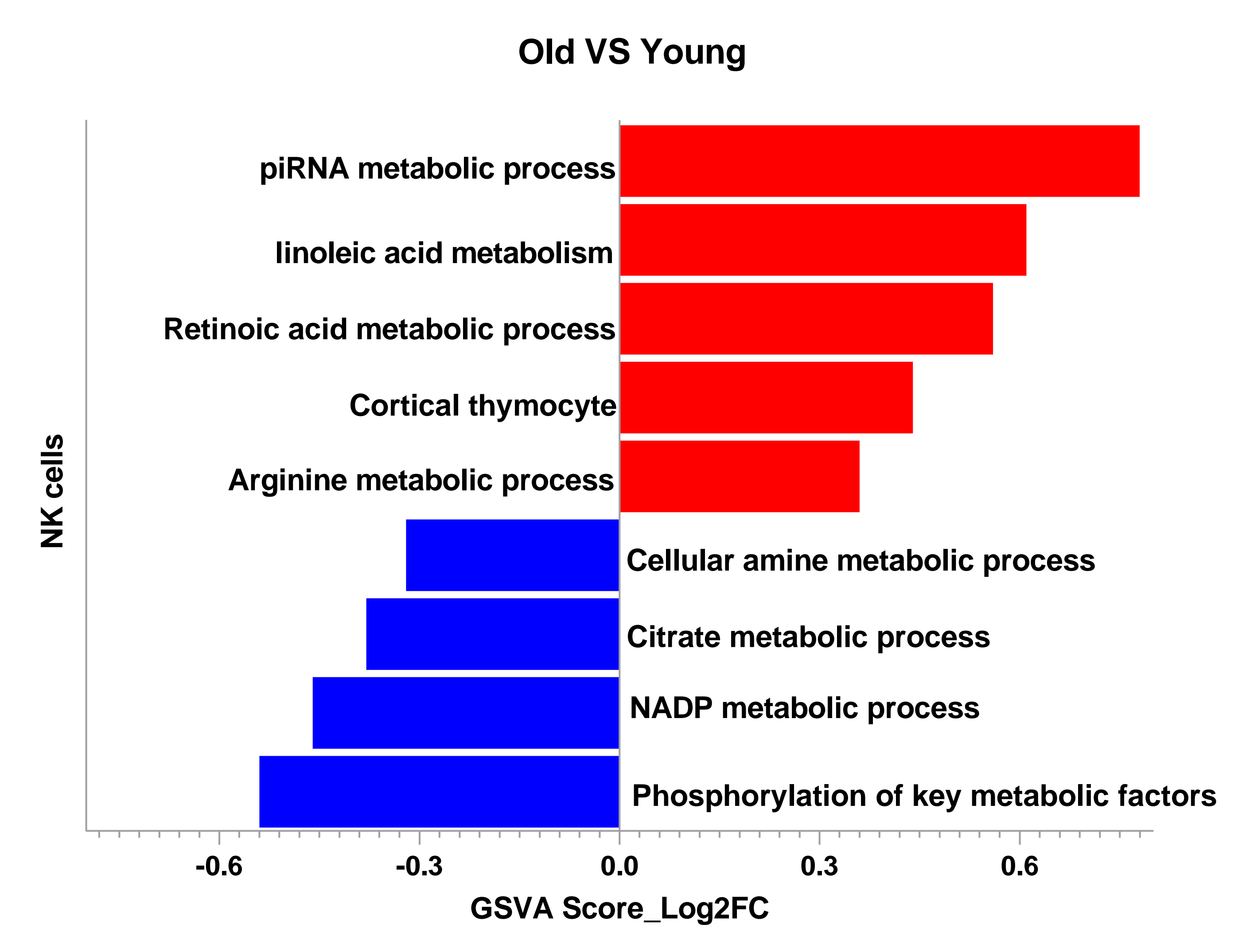

C

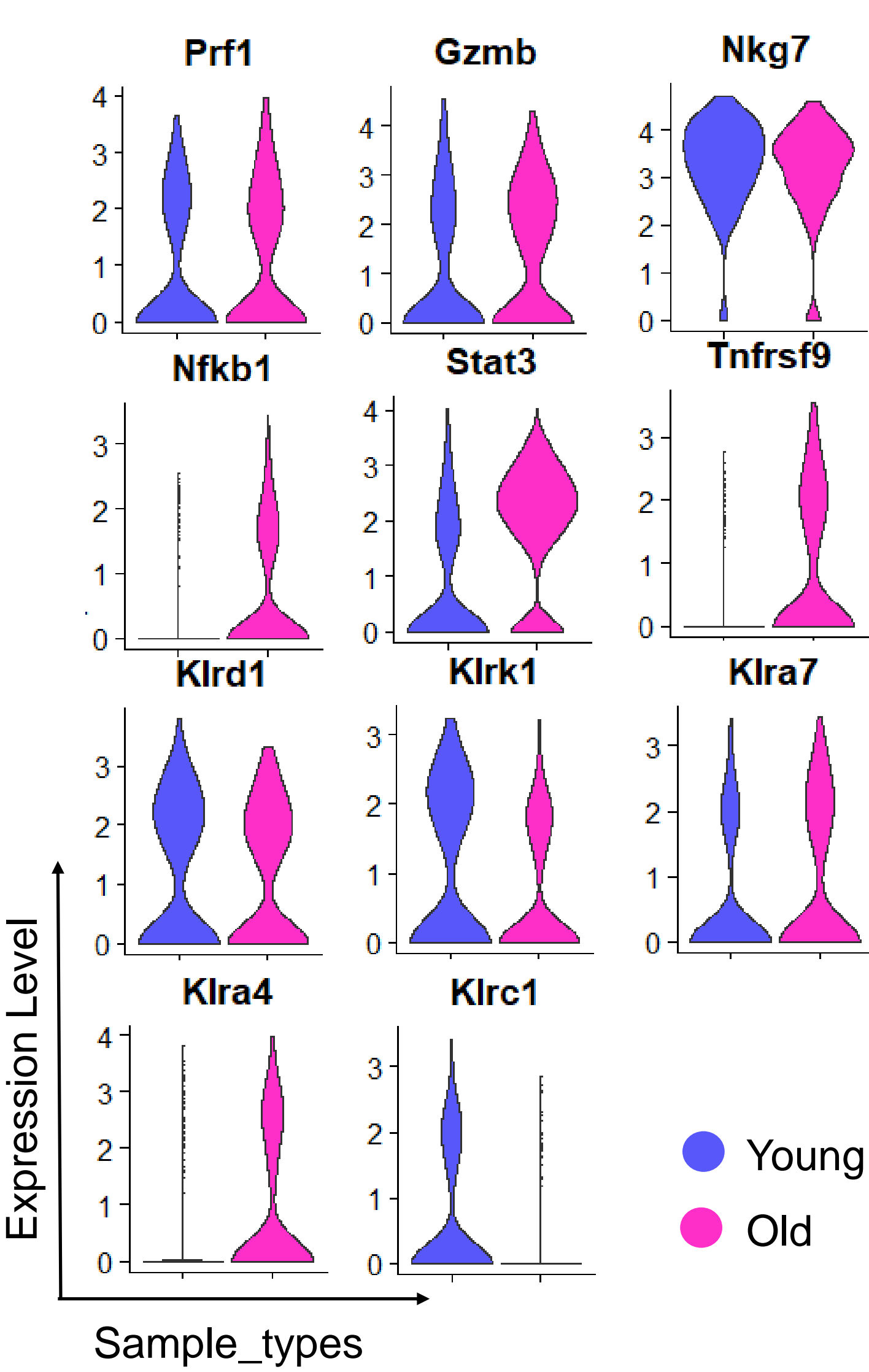


A
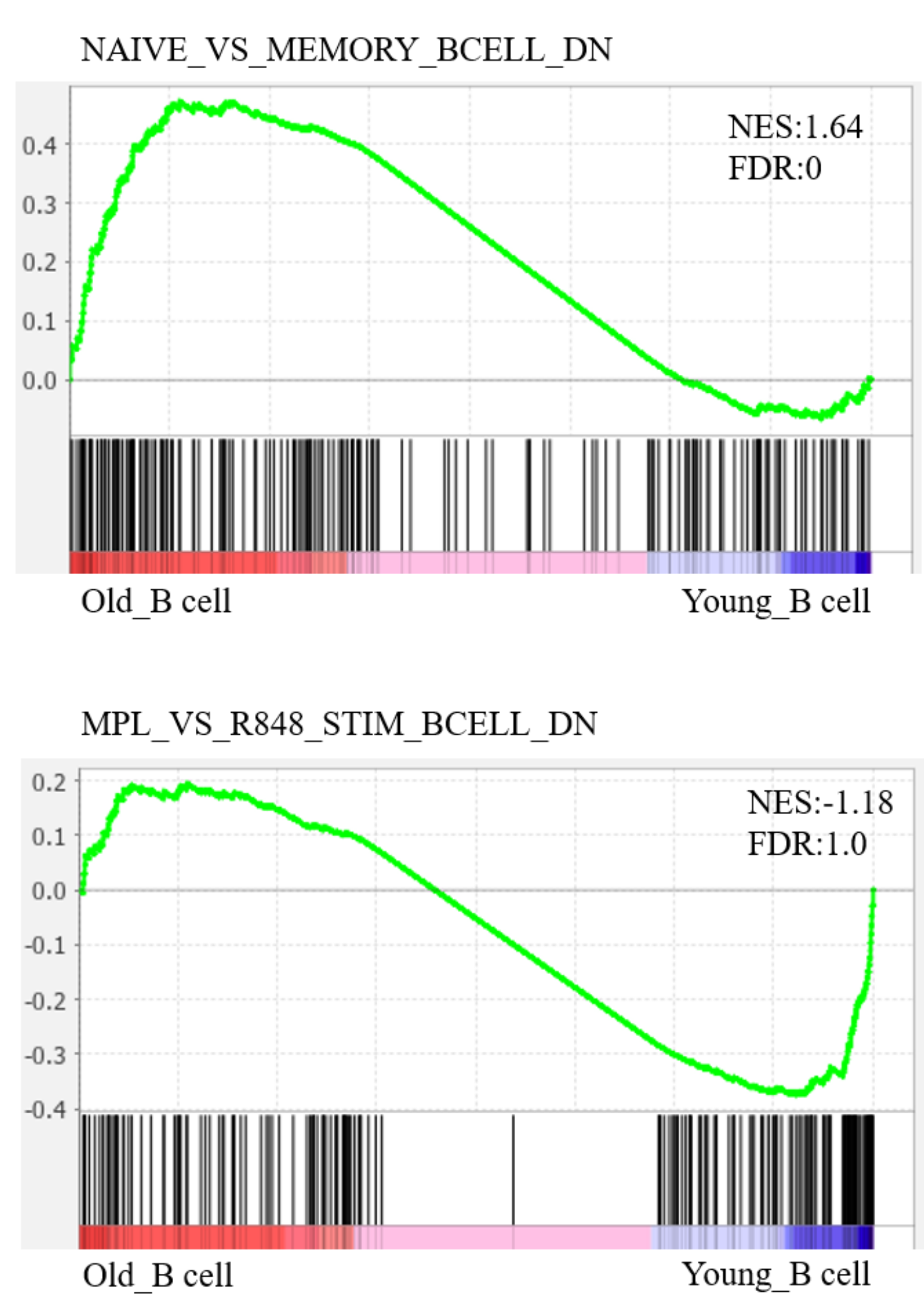

B

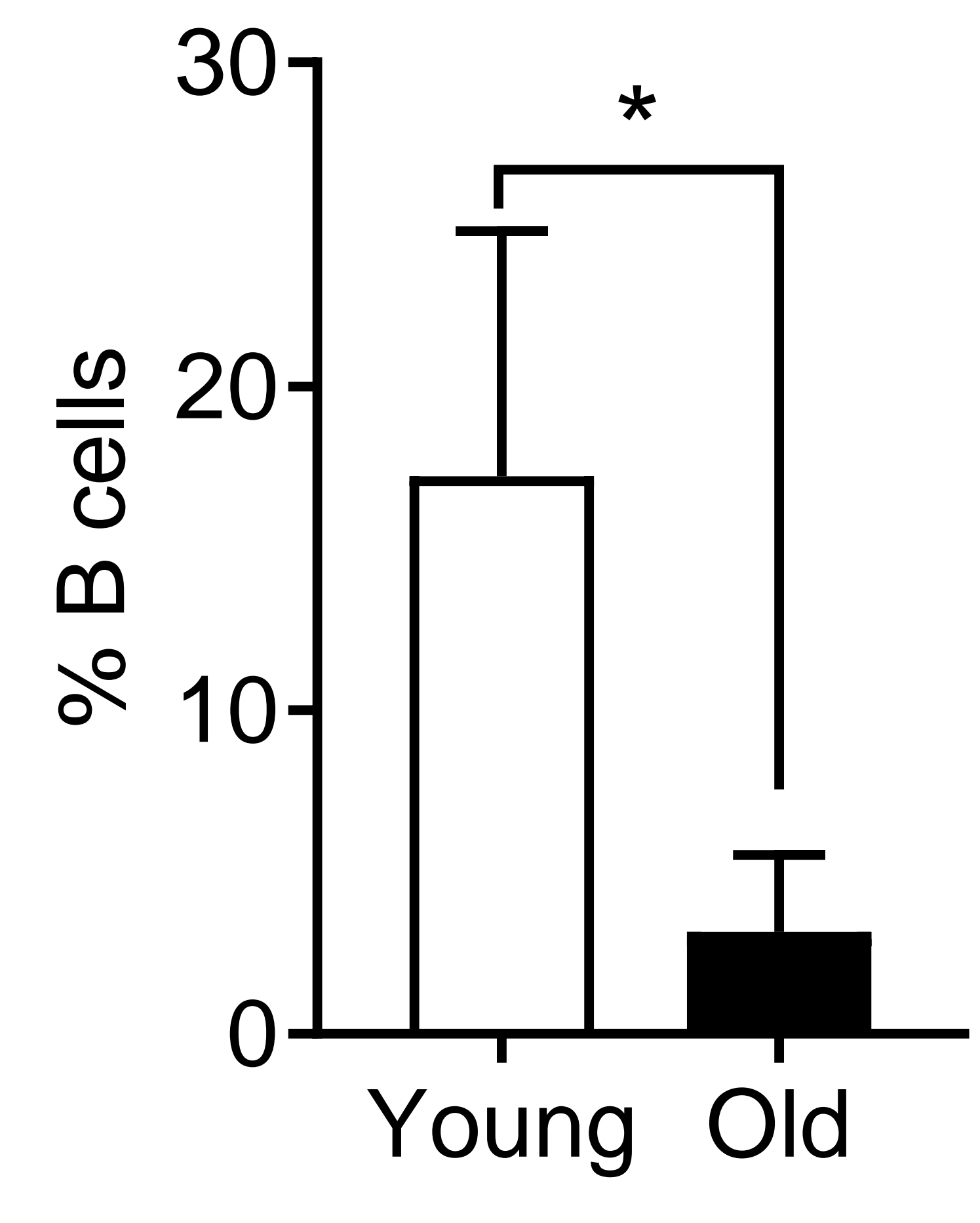

C

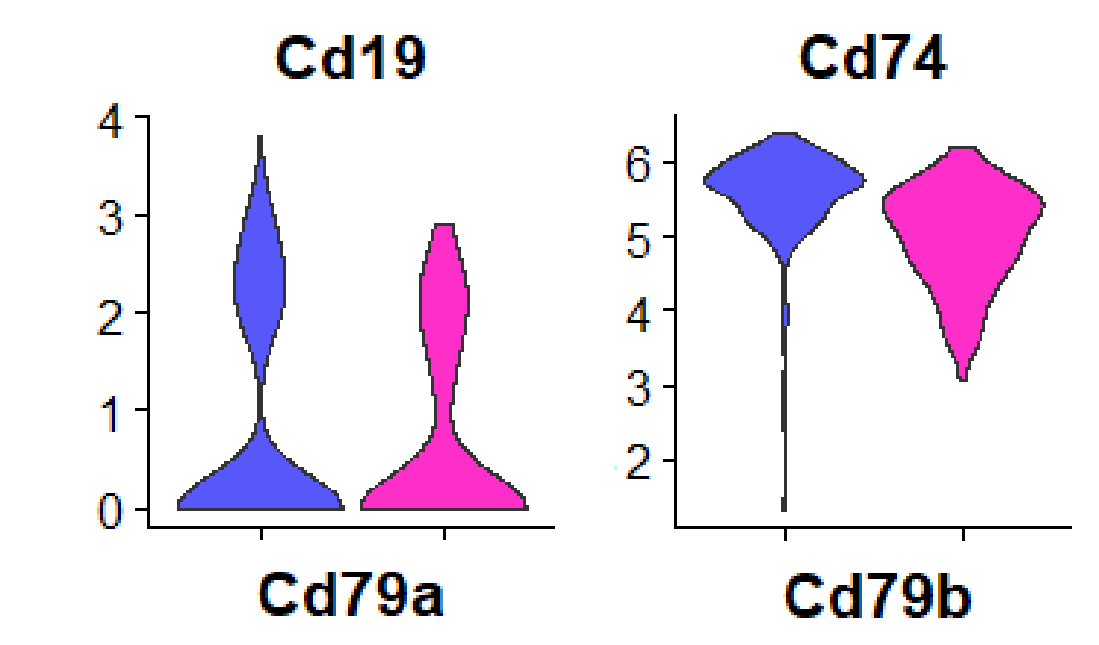

$\underset{\text { Ly6a }}{2}$

$\underbrace{C d 79 b}_{C d 44}$

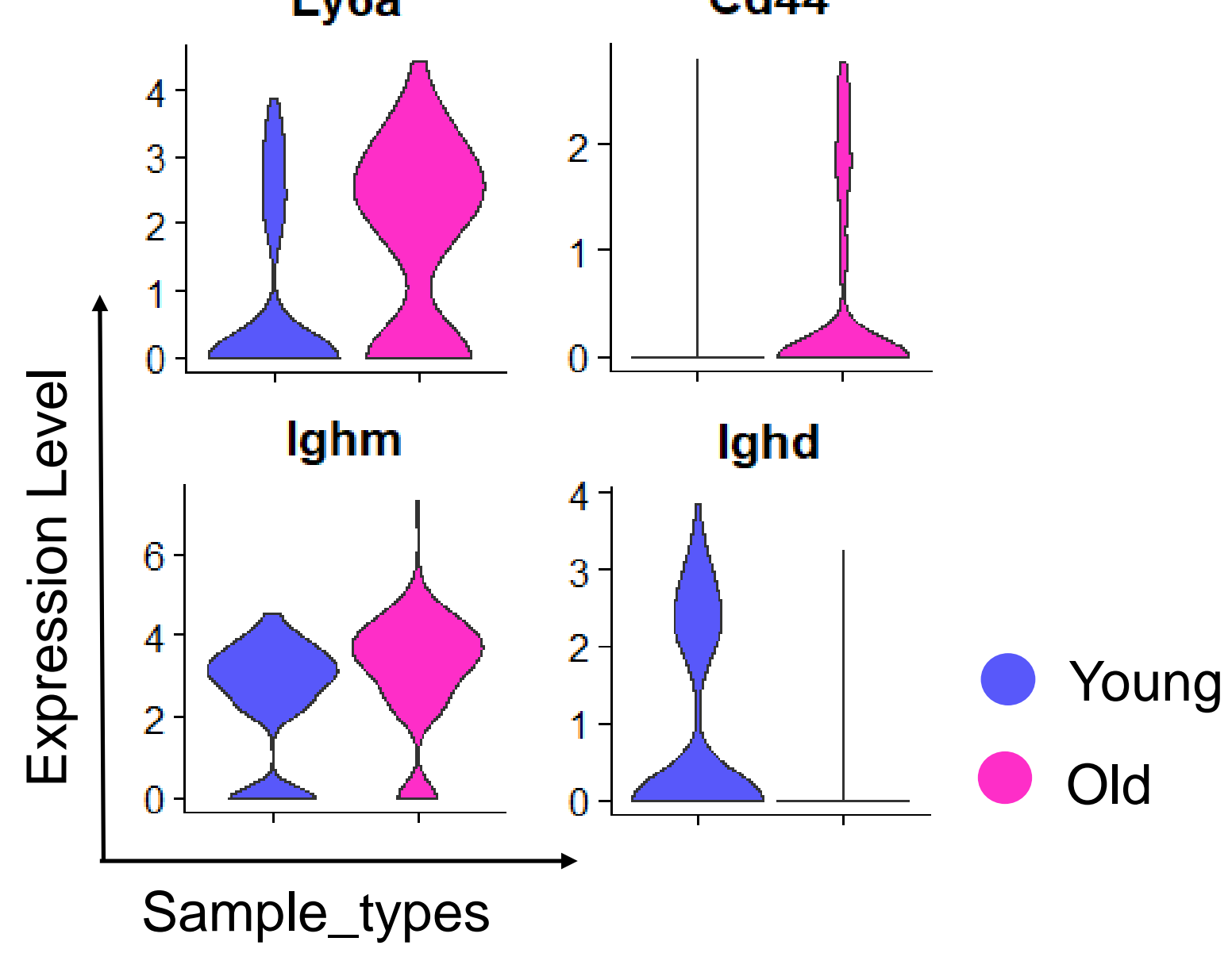

